\title{
LIMIT ON THE MUON NEUTRINO MAGNETIC MOMENT AND A MEASUREMENT OF THE CCPIP TO CCQE CROSS SECTION RATIO
}

\author{
A Dissertation \\ Submitted to the Graduate Faculty of the \\ Louisiana State University and \\ Agricultural and Mechanical College \\ in partial fulfillment of the \\ requirements for the degree of \\ Doctor of Philosophy \\ in
}

The Department of Physics And Astronomy

by

Serge Ouedraogo

B.S. in Physics, University of Arkansas at Little Rock, 2001

M.S., Louisiana State University, 2004

December 2008 
In loving memory of My father,

Joseph Ouedraogo 


\section{Acknowledgments}

It is a pleasure to express my gratitude and appreciation to my advisor Professor William J. Metcalf for his professional advice, critical comments and patience in directing this thesis. His careful reading of this thesis and his insightful suggestions were helpful to complete this work. I would like to acknowledge my deep appreciation to Professor Emeritus Richard Imlay for major contributions presented in this thesis. I would like to thank him for the many trips he took to Baton Rouge to direct the elastic scattering analysis and for patiently reading this dissertation countless times.

I am specially grateful to the LSU postdocs who helped shape my understanding of neutrino physics. First, I wish to thank Dr. Myung Kee Sung from whom I learned how to use various computing analysis tools. I also wish to thank Dr. Morgan Wascko for guiding me through my first hardware experience, my first data analysis and for the many advices he kindly provided. Finally I wish to thank Dr. Jarosław Novak for many enlightening discussions and also for reading through my thesis when it was still in its cryptic form.

I wish to thank the MiniBooNE collaboration through the spokespersons of Dr. William C. Louis III, Prof. Janet Conrad, Dr. Steve Brice and Dr. Richard Van De Water. They provided wonderful leardership and fostered an excellent research environment. I also want to specially thank Dr. Yong Liu for initiating me to his reconstruction algorithm and for reproducing the elastic scattering Monte Carlo sample which was critical for the analysis presented in this thesis. I would also like to thank my fellow graduate students past and pressents at MiniBooNE. I wish to especially acknowledge Alexis Aguilar-Arevalo, Chris Cox, Teppei Katori, Kendall Mahn and Denis Perevalov. 
I am deeply thankful to my sister Eva and brothers Clifford and Olivier for their constant support in various forms. My heartfelt thanks goes to my parents Joseph and Yvette who have made enormous sacrifices for me to in this position. I've always regret that my father has not lived long enough to see this day.

My stay in Baton Rouge has been made easier thanks to Mr Roland and Barbara Smith who made me feel like home. I am deeply grateful for all the supports and encouragements they provided to both my wife and I and for being there for us at all time.

Finally, I would like to thank my wife Moon. Without her constant love, support and patience I would not have found the strength to go all the way. Thank you for sharing with me all the hard time and good time of a graduate student life. 


\section{Table of Contents}

DEDicAtion $\ldots \ldots \ldots \ldots \ldots \ldots \ldots \ldots \ldots \ldots \ldots \ldots \ldots$ ii

ACKNOWLEDGMENTS $\ldots \ldots \ldots \ldots \ldots \ldots \ldots \ldots \ldots$........... iii

List of TABLES $\ldots \ldots \ldots \ldots \ldots \ldots \ldots \ldots \ldots \ldots \ldots$ ix

List of Figures $\ldots \ldots \ldots \ldots \ldots \ldots \ldots \ldots \ldots \ldots$ xiv

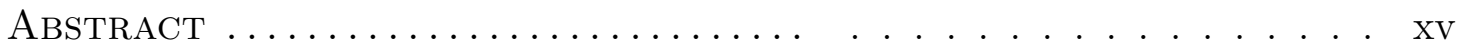

1 Introduction . . . . . . . . . . . . . . . . . . . . . . 1

1.1 Overview of the G-W-S Model . . . . . . . . . . . . . . 2

1.2 Neutrino Properties in the G-W-S Model . . . . . . . . . . . . 5

1.2.1 Chirality and Helicity . . . . . . . . . . . . . 5

1.2.2 Charge Conjugation and Parity . . . . . . . . . . 6

1.3 Origin of Mass . . . . . . . . . . . . . . . . . . . . . . 7

1.3.1 Fermion Mass . . . . . . . . . . . . . . . . . 7

1.3.2 Neutrino Mass . . . . . . . . . . . . . . . . . . . 9

1.4 Electromagnetic Properties of Neutrinos . . . . . . . . . . . . . . . 11

1.4.1 Charge Radius . . . . . . . . . . . . . . . . 12

1.4.2 Neutrino Magnetic Moment . . . . . . . . . . . . . . 13

1.5 Neutrino-Nucleon Scattering . . . . . . . . . . . . . . . . 16

1.5.1 Resonant Production . . . . . . . . . . . . . . . 16

1.5.2 Coherent Production . . . . . . . . . . . . . . . . 17

1.5.3 Motivation of the $\mathrm{CC} \pi^{+}$Cross Section Study . . . . . . . . . 17

1.5.4 Resonant Charged Current Single $\pi^{+}$Cross Section . . . . . 18

1.6 Plan of the Thesis . . . . . . . . . . . . . . . . . . . . . . 19

2 MiniBoone Experiment ..................... . . 20

2.1 Neutrino Beam . . . . . . . . . . . . . . . . . . 21

2.1.1 Proton Source . . . . . . . . . . . . . . 21

2.1.2 Horn and Target . . . . . . . . . . . . . . . 22

2.1.3 Decay Region . . . . . . . . . . . . . . . . . 23

2.1.4 Detector ..................... . . 24

2.2 Data Acquisition . . . . . . . . . . . . . . . . . . . . . . . . . . . . . . . . . 25

2.3 Trigger Sytem . . . . . . . . . . . . . . . . . . . . 29

2.4 Calibration System . . . . . . . . . . . . . . . 30

2.4.1 Laser Calibration System . . . . . . . . . . . . . . 30

2.4.2 PMTs Calibration . . . . . . . . . . . . . . . . . 32

2.4.3 Cosmic Ray Muon Calibration System . . . . . . . . . . 33 
3 Experiment Simulation . . . . . . . . . . . . . . . . . . . . . 39

3.1 Overview . . . . . . . . . . . . . . . . . . . . . . . . . 39

3.2 Interactions in the Beryllium Target . . . . . . . . . . . . . 39

3.2 .1 Inelastic Interactions . . . . . . . . . . . . . . . . . . . 39

3.2.2 Elastic And Quasi-Elastic Interactions . . . . . . . . . . . 42

3.3 Electromagnetic Processes . . . . . . . . . . . . . . . . . 43

3.4 Neutrino Flux . . . . . . . . . . . . . . . . . . . . . . . . 44

3.5 Neutrino Cross Section Model . . . . . . . . . . . . . . . . 44

3.5.1 Simulation of Neutrino Electron Scattering . . . . . . . . 45

3.5.2 Simulation of Charged Current Quasi-Elastic Interaction . . 47

3.5.3 Simulation of Charged Current Single Pion production . . . 48

3.5.4 Nuclear Effects . . . . . . . . . . . . . . . . . . . . . . 49

3.6 Detector Simulation . . . . . . . . . . . . . . 50

3.6.1 Light Production in the Detector . . . . . . . . . . 50

3.6.2 Light Transmission . . . . . . . . . . . . . . . . . 51

3.6 .3 Output of Detector Simulation . . . . . . . . . . . . 54

4 Reconstruction Algorithm And Particle Identification ... 55

4.1 Event Reconstruction . . . . . . . . . . . . . . . . . 55

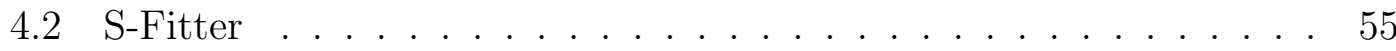

4.2.1 Fast Fit: Time Likelihood Fit . . . . . . . . . . . 55

4.2 .2 Full Fit . . . . . . . . . . . . . . . . . . . 57

4.2 .3 Flux Fit . . . . . . . . . . . . . . . . . 58

4.2 .4 Track Reconstruction . . . . . . . . . . . . . . . . . . 58

4.3 P-Fitter . . . . . . . . . . . . . . . . . . . . 59

4.4 Particle Identification (PID) . . . . . . . . . . . . . . . 60

4.4.1 Track-Based Particle Identification . . . . . . . . . . . . . 61

4.4 .2 Boosting Decision Tree . . . . . . . . . . . . . . . . . . 62

4.4.3 PID Variables for Low-Energy $\nu_{\mu}$-e Events . . . . . . . . . 64

5 Low Energy Elastic Scattering Events . . . . . . . . . 65

5.1 Overview of the Event Samples . . . . . . . . . . . . 66

5.1 .1 Data Sample . . . . . . . . . . . . . . . . . 66

5.1 .2 Simulated Beam-Induced Events . . . . . . . . . . . . . 66

5.1 .3 Beam Off Events . . . . . . . . . . . . . . . . . . . 67

5.2 Low Energy $\nu_{\mu}$ e Event Selection . . . . . . . . . . . . . . . 67

5.3 Study of the Cosmic Ray Background . . . . . . . . . . . . . . . . 69

5.3.1 Description of The Past Time Cut . . . . . . . . . . . 69

5.4 The Beam Related Background . . . . . . . . . . . . 73

5.4 .1 In-tank Events . . . . . . . . . . . . . . . . 74

5.4 .2 Dirt Events . . . . . . . . . . . . . . . . . . . . . 75

5.5 Final Event Sample And Normalization of the Background: Counting Method . . . . . . . . . . . . . . . 78 
5.6 Observation of An Excess of Low Energy Events in the Forward Direction . . . . . . . . . . . . . . . . . . 79

5.7 Extracting Signal From $\operatorname{Cos} \theta$ Distribution . . . . . . . . . . 80

5.8 Significance of the Excess _ . . . . . . . . . . . . . 87 5.8.1 Significance For The Counting Method . . . . . . . . . . 87

5.8.2 Significance For the $\operatorname{Cos} \theta$ Fitting Procedure . . . . . . . 88

5.9 Reconstruction Bias . . . . . . . . . . . . . . . . . . . . . 91

5.10 Results . . . . . . . . . . . . . . . . . . . . . . . . 92

6 High Energy Elastic Scattering Events . . . . . . . . . . 95

6.1 High Energy $\nu_{\mu}$ e Event Selection . . . . . . . . . . . . . . 95

6.2 Dirt Event Rate In the Data Sample . . . . . . . . . . . . . . 97

6.3 Analysis Based On PID Variables From The Boosting Decision Tree (BDT) Algorithm . . . . . . . . . . . . . . . . . . . . 97

6.4 Analysis Based On PID Variables From the Track-Based Algorithm (TBA) . . . . . . . . . . . . . . . . . . . 102

6.5 Combined Analysis . . . . . . . . . . . . . . . . 105

6.6 Summary . . . . . . . . . . . . . . . . . . . 107

7 Measurement of CC $\pi^{+}$Cross Section . . . . . . . . . . 109

7.1 Procedure . . . . . . . . . . . . . . . . . . . . . . 110

7.2 Event Selection . . . . . . . . . . . . . . . . . 111

$7.2 .1 \quad \mathrm{CC} \pi^{+}$Event Selection . . . . . . . . . . . . . . 112

7.2 .2 CCQE Event Selection . . . . . . . . . . . . . . . . 113

7.3 Systematic Errors . . . . . . . . . . . . . . . . . . . . . . 114

7.3.1 Cross Section and Flux Uncertainties . . . . . . . . . . 115

7.3.2 Optical Model Uncertainties . . . . . . . . . . . . . . . . 115

7.4 Cross Section Ratio Measurement . . . . . . . . . . . . . . . 116

7.4.1 Result Overview . . . . . . . . . . . . . . . . 116

7.4 .2 Results . . . . . . . . . . . . . . . . . . . . 117

7.4 .3 Summary . . . . . . . . . . . . . . . . . . . . . 119

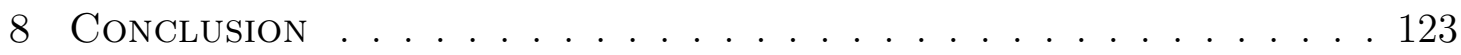

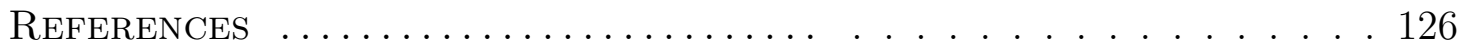

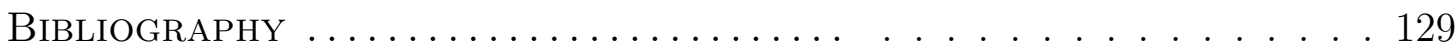

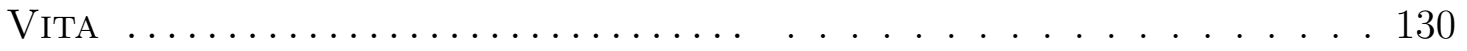




\section{List of Tables}

2.1 Internal bit triggers and their thresholds. . . . . . . . . . . . . . 30

2.2 Flask positions in the MiniBooNE detector. . . . . . . . . . . . . . . 32

2.3 Scintillator cube positions in beam coordinates ( $\mathrm{z}$ along beam, y toward tophat, $\tan (\phi)=\mathrm{z} / \mathrm{x}) . \ldots \ldots \ldots . \ldots . \ldots . \ldots 36$

3.1 Sanford-wang parameters for $\pi^{+}, \pi^{-}, K^{0}$ production . . . . . . . . . 40

3.2 $\mathrm{K}^{+}$production data used for the Feynman scaling fits. Data with 1.2 $\mathrm{GeV} / \mathrm{c}<\mathrm{p}<5.5 \mathrm{GeV} / \mathrm{c}$ are used. The data from Vorontsov were not used because of a large normalization offset compared to the data from the other sources. . . . . . . . . . . . . . . . . 42

3.3 Feynman scaling parameters for $\mathrm{K}^{+}$production. . . . . . . . . . . . . . 42

3.4 MiniBooNE Geant4 beam Monte Carlo neutrino flux production modes. 46

5.1 A summary of all the cuts used after the precuts listed in section 5.2. The motivation behind each cut and the effectiveness of the cut for each data sample are given. . . . . . . . . . . . . . . 78

5.2 This table shows the observed number of events after each cut is applied sequentially. . . . . . . . . . . . . . . . . . . 78

5.3 A summary of the different event samples used for the analysis is presented. The second column shows the number of events that have passed the precuts. The third column is the number of events after all cuts were applied, while the next column shows the corresponding protonson-target for each sample. Column 5 displays the expected rate for the $\nu$ elastic scattering plus all the significant backgrounds together with the observed number of data event all corresponding to $4.67 \mathrm{E} 20$ pot. .

5.4 A table comparing the data event rate to the expected event rate. We observe an excess of 18.2 events above the expected signal and background. . . . . . . . . . . . . . . . .

6.1 Survival fraction of background events, signal events and data after the PID cuts and the $\pi^{0}$ mass cut were applied sequentially. . . . . . . . . 100 
6.2 The table summarizes the number of signal, in-tank and dirt events for 5.68E20 POT. The cuts applied are the precuts, rtowall, the cuts on Boosting PID variables and the $\pi^{0}$ mass. . . . . . . . . . . . . 100

6.3 The table summarizes the number of signal and background events for 5.68E20 POT. The cuts applied are the precuts, rtowall and the cuts on Boosting PID variables. . . . . . . . . . . . . . . . . . . 102

6.4 Survival fraction of background events, signal events and data after the PID cuts and the $\pi^{0}$ mass cut were applied sequentially. . . . . . . 103

6.5 The table summarizes the number of data, signal and background events for 5.68E20 POT. The cuts applied are the precuts, rtowall and the cuts on TBA PID variables. . . . . . . . . . . . . . . . . . 105

6.6 The table summarizes the rate of data, signal and background events for 5.68E20 POT. The cuts applied are the precuts, rtowall and the cuts on the TBA PID variables. . . . . . . . . . . . . . . 105

6.7 A table summarizing the number of In-tank, dirt and elastic scattering events at different intervl of $\cos \theta \ldots \ldots$. . . . . . . . 107

6.8 The table summarizes the number of data, signal and background events for 5.68E20 POT. The cuts applied are the precuts, rtowall and the cuts on the TBA PID variables. . . . . . . . . . . . . . . 107

7.1 MonteCarlo event compositions after the $\mathrm{CC} \pi^{+}$cut requirements. . . . 113

7.2 MonteCarlo event compositions after the CCQE cut requirements. . . . 114 


\section{List of Figures}

2.1 A schematic representation of the MiniBooNE beamline and detector . 21

2.2 Booster fixed target facility with the $8 \mathrm{GeV}$ beamline. . . . . . . . . . 21

2.3 A schematic representation of the MiniBooNE horn. The beryllium target is located inside the focusing magnet (green). . . . . . . . . . . . 22

2.4 MiniBooNE detector showing inner volume and outer veto shell . . . . 25

2.5 A picture of a 8-inch PMT used in the MiniBooNE experiment . . . . . 26

2.6 PMT charge and time signals. The anode pulse is given by $\mathrm{V}_{p m t}$ and the integrated charge by $\mathrm{V}_{q}$. The Discrimator digial pulse and the $\mathrm{V}_{t}$ time ramp are started when the anode pulse crosses a preset threshold.

2.7 Schematic diagram of data acquisition electronics for a single PMT channel. . . . . . . . . . . . . . . . . . . . 28

2.8 MiniBooNE laser calibration system . . . . . . . . . . . . . . . 31

2.9 A schematic diagram of the muon tracker and a scintillation cube . . . 34

2.10 Position resolution of the four planes that constitute the muon tracker

2.11 Muon energy determined by the reconstruction vs. cube range energy calculated from the muon path obtained from the muon tracker and the scintillator cubes inside the tank. Data is shown by points, Monte Carlo is the solid histogram. . . . . . . . . . . . . . . .

2.12 Corrected angle distributions of tank PMT hits for stopping muon events in the six deepest cubes. The event vertex and time are measured using the cubes and muon tracker. Data is shown by points, Monte Carlo is the solid histogram. . . . . . . . . . . . . . . . . .

3.1 A plot showing HARP results for $\mathrm{pBe} \rightarrow \pi^{+} \mathrm{X}$ interaction. The SanfordWang function is the red curve and the HARP data are the red points. The blue curves represent the uncertainties. . . . . . . . . . . . .

3.2 Feynman scaling parametrization (solid curve), error bands (dashed curve), and data (points) for the $\mathrm{K}^{+}$production. . . . . . . . . . 
3.3 Predicted neutrino flux in the MiniBooNE detector for each neutrino

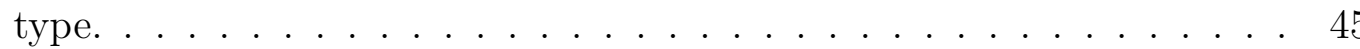

3.4 Photon attenuation rate in MiniBooNE mineral oil. Shown in this plot are the different components of the attenuation rate: scattering, absorption and fluorescence. . . . . . . . . . . . . . . . 52

4.1 Hit topologies of electrons (left), muons (middle) and NC $\pi^{0}$ (right) events in the MiniBooNE detector. . . . . . . . . . . . . . . . 61

4.2 On the left, the distribution of $\log \left(\mathrm{L}_{e} / \mathrm{L}_{\mu}\right)$ as a function electron energy is used to separate a Monte Carlo simulation of $\nu_{e}$ CCQE events from a simulation of $\nu_{\mu} \mathrm{CCQE}$ events. On the right, a simulation of $\nu_{e} \mathrm{CCQE}$ events is separated from a simulation of $\nu_{\mu} \mathrm{NC} \pi^{0}$ events using the PID $\log \left(\mathrm{L}_{e} / \mathrm{L}_{\pi}\right)$ as a function of the electron energy. The values of the cuts were selected to optimized the oscillation sensitivity. . . . . . . . . .

5.1 The time distribution of the CCQE first subevent is shown to be between 4550 and $6250 \mathrm{~ns}$. We expect this situation to be identical for

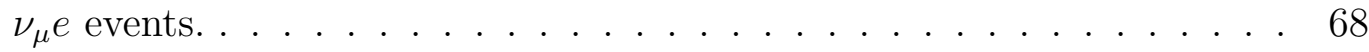

5.2 This plot shows the time distribution of events passing all the precuts except the beam time cut. The exponential fall off indicates most of these are Michel electrons with their parent muon outside the window.

5.3 On the left is shown the past event time distribution for strobe events passing the precuts. Fitting the distribution to an exponential yields a decay lifetime of $1.87 \pm 0.3 \mu \mathrm{sec}$, consistent with the muon lifetime. The energy distribution of subevents with activity in the $10 \mu$ sec interval before the window opens is displayed on the right, and is consistent with the energy distribution of Michel events. . . . . . . . . . . .

5.4 This plot is a display of the $\Delta t$ distribution for subevents for which we failed to find an activity in the tank $10 \mu$ sec before the beam window opened. . . . . . . . . . . . . . . . . 72 
5.5 Left: The PID variable shows a good separation between electron candidates (blue and red) from beam related background (green). The cyan histogram is a Monte Carlo simulation of $\nu_{\mu}$ e events, while the red histogram shows electrons from cosmic events that we remove with the past time cut. Events in this plot have passed the precuts requirement. Right: The scintillation light fraction distribution for dirt (magenta), electroweak (blue), NCEL (green) and cosmic (red) events are shown. In this plot, the events were required to pass both the precuts and the PID cut. . . . . . . . . . . . . . . . . . . . . . . .

5.6 A cartoon representation of a photon from the decay of a $\pi^{0}$ event produced from the interaction of neutrinos with the dirt outside the detector. . . . . . . . . . . . . . . . . 75

5.7 Rtowall distribution for events passing the precuts. . . . . . . 76

5.8 The veto hits distributions for data events (black points) and different Monte Carlo event samples are shown. Dirt events are in magenta, cosmic events in red, electroweak events are in blue and Michel electrons from $\mathrm{CC} \pi^{+}$are in light green. . . . . . . . . . . . . . . . . . . . 77

5.9 Shown in these plots are the number of candidate electron events as a function of $\cos \theta$ (top) and recoil energy (bottom). The points represent the data with statistical errors, while the colored histograms are the different sources of electrons normalized to the data protons-on-target. The blue histogram shows the expected signal events. The green and red histograms show respectively the beam related background and the cosmic related background. . . . . . . . . . . . . . . . . . . .

5.10 Data and Monte Carlo distribution of radius (top) and time (bottom) for events passing all the cuts. The points represent the data with statistical errors, while the colored histograms are the different sources of electrons normalized to the data protons-on-target. The blue histogram shows the expected signal events. The green and red histograms show respectively the beam related background and the cosmic related background.

5.11 Data and Monte Carlo distribution of spatial coordinates for events passing all the cuts. The points represent the data with statistical errors, while the colored histograms are the different sources of electrons normalized to the data protons-on-target. The blue histogram shows the expected signal events. The green and red histograms show respectively the beam related background and the cosmic related background. . . . 
5.12 Data and Monte Carlo distribution of variables used in the event selection. The points represent the data with statistical errors, while the colored histograms are the different sources of electrons normalized to the data protons-on-target. The blue histogram shows the expected signal events. The green and red histograms show respectively the beam related background and the cosmic related background. . . . . . . . . .

5.13 The top plot shows the $\cos \theta$ distribution of simulated $\nu_{\mu}$ e events fitted with a function that will be used to construct the likelihood function. The two bottom plots are the angular distribution of the cosmic background (left) and beam background (right). Within statistics, those plots are consistent with being flat. . . . . . . . . . . . . . .

5.14 Angular distribution of the electroweak events in red and electroweak events reweighted by $1 / \mathrm{T}$ in black. . . . . . . . . . . . . . . .

5.15 Angular distribution of Michel electrons. The flatness of the distribution indicates no bias in reconstruction. . . . . . . . . . . . . . . .

5.16 Gaussian distribution for 10000 fake experiments with $\mathrm{f}=12.7 \times 10^{-10}$. The red line is at 15.3 showing that $90 \%$ of the time, we would see an excess of at least 15.3 events if the magnetic moment were $12.7 \times 10^{-10} \mu_{\nu} .93$

6.1 Energy distribution for events passing the precuts, except the energy cut. The data events are shown in black dots, the $\nu_{\mu}$ e events in blue color and the in-tank in green color. . . . . . . . . . . . . . 96

6.2 Left: Rtowall distribution for data events (black), elastic scattering events (blue) and dirt events (red) passing the precuts. Right: Same distribution after a rtowall cut was applied.The distributions are normalized to unit area. . . . . . . . . . . . . . . . . . . . .

6.3 The ELMU PID distribution for events that passed the precuts and the rtowall cut is shown on the left. On the right is the ELPI distribution for events passing the precuts, rtowall and the ELMU cut. The $\pi^{0}$ mass distribution of events passing the precuts, rtowall and both PID cuts is shown in the bottom. In all of these plots the blue (green) curve shows the signal (background) events. The data are in black with statistical error. . . . . . . . . . . . . . . . . . . .

6.4 Angular distribution, energy distribution and radius distribution events passing the precuts, the rtowall cuts and the BDT pid cuts. The data events are shown in black dots, the $\nu_{\mu}$ e events in blue color and the in-tank in green color. . . . . . . . . . . . . . . . . . 101 
6.5 The likeemu (left) and the likeepi (right) distributions for events passing the precuts are shown. The blue (green) curve shows the signal (background) events. The data are in black with statistical error. . . . . 103

6.6 Angular distribution, energy distribution and radius distribution for events passing the precuts, the rtowall cuts and the TBA pid cuts. The data events are shown in black dots, the $\nu_{\mu}$ e events in blue color and the in-tank in green color . . . . . . . . . . . . . . . . . . 104

6.7 Angular distribution for events passing the precuts, rtowall and the BDT PID cuts. . . . . . . . . . . . . . . . . . 106

7.1 Right:The $\mathrm{CC} \pi^{+}$to CCQE ratio with cross section and flux uncertainties. Left: Ratio fractional error from cross section and flux uncertainties.116

7.2 Left: The $\mathrm{CC} \pi^{+}$to CCQE cross section ratio with optical model uncertainties only. Right: The ratio fractional errors from the optical model only. . . . . . . . . . . . . . . . . . . . 117

7.3 Reconstructed neutrino energy for neutrino mode data for the $\mathrm{CC} \pi^{+}$ (left) and CCQE (right). For $\mathrm{CC} \pi^{+}$the Stancu fitter (black) is compared with two versions of one-track fitter, original (old - green) and with muon energy adjustment (new - red). . . . . . . . . . . . . 118

7.4 The signal fraction (left) and cut efficiency (right) for the $\mathrm{CC} \pi^{+}$sample for three fitters. . . . . . . . . . . . . . . . . . . . 120

7.5 The unsmearing matrices for the three fitters . . . . . . . . . . . . 121

7.6 Top: The $\mathrm{CC} \pi^{+}$to CCQE ratio for stancu fitter (black curve), the P-fitter without energy correction (green) and with energy correction (red). Bottom: the left plot is the $\mathrm{CC} \pi^{+}$to $\mathrm{CCQE}$ ratio distribution using the P-fitter including all the sources of errors. The right plot shows the fractional errors after taking into account all sources of errors. 122

8.1 The $\mathrm{CC} \pi^{+}$to $\mathrm{CCQE}$ ratio for MiniBooNE, K2K and ANL . . . . . . . 125 


\section{Abstract}

A search for the muon neutrino magnetic moment was conducted using the MiniBooNE low energy neutrino data. The analysis was performed by analyzing the elastic scattering interactions of muon neutrinos on electrons. The analysis looked for an excess of elastic scattering events above the Standard Model prediction from which a limit on the neutrino magnetic could be set. In this thesis, we report an excess of $15.3 \pm 6.6$ (stat) \pm 4.1 (syst) $\nu_{\mu} e$ events above the expected background. At $90 \%$ C.L., we derived a limit on the muon neutrino magnetic moment of $12.7 \times$ $10^{-10} \mu_{B}$. The other analysis reported in this thesis is a measurement of charged current single pion production $\left(\mathrm{CC} \pi^{+}\right)$to charged current quasi elastic (CCQE) interactions cross sections ratio. This measurement was performed with two different fitting algorithms and the results from both fitters are consistent with each other. 


\section{Chapter 1}

\section{Introduction}

In 1930, W. Pauli introduced the neutrino in order to solve the crisis that resulted from the continuous electron spectrum accompanying nuclear beta decay CT:Pauli:1930. Since the process appeared to violate both the conservation of energy and angular momentum, Pauli suggested that together with the electron, a new particle was emitted. He postulated this new particle, the neutrino, would have no electric charge, would have a mass of order or less than the electron mass and would carry half a unit of angular momentum in order to balance angular momentum in the nuclear $\beta$-decay. For energy to be conserved, the new particle would also carry a fraction of the energy released. In 1934, Enrico Fermi formulated a theory of the weak interaction to describe the decay process, but experimental evidence for the existence of neutrino was not obtained until 1956 when Reines and Cowan reported their observation in an experiment at the Savannah River reactorPhysRev.90.492.2. In his paper [1], Fermi described the interaction by introducing a four-Fermion Hamiltonian density:

$$
\mathcal{H}_{\text {weak }}=\frac{G_{F}}{\sqrt{2}}\left(\bar{\psi}_{p} \gamma_{\mu} \psi_{n}\right)\left(\bar{\psi}_{e} \gamma_{\mu} \psi_{\nu}\right)
$$

where $\psi_{p}, \psi_{n}, \psi_{e}, \psi_{\nu}$ denote respectively the fields of the proton, neutron, electron and neutrino.

The Hamiltonian shown in equation (1.1) is a good approximation of the weak interaction, but in the following decades it was found that:

- the fundamental fields in the Hamiltonian are quark fields and not hadronic fields. 
- the weak current is described by both vector $\left(\gamma_{\mu}\right)$ and axial vector $\left(\gamma_{\mu} \gamma_{5}\right)$ components.

With these modifications, the four-Fermi theory provided a good description of observed low energy weak interaction processes, but it led to a cross section for neutrino-lepton scattering that grows with energy and eventually violates the unitary bound. In the process of resolving this issue, as well as others not mentioned in this thesis, Glashow, Weinberg and Salam introduced a new theory that unifies weak interactions and electromagnetic interactions into one theoretical model called the G-W-S model. In this model, the weak interaction consists of both a charge current (CC) interaction, in which the electric charge of the interacting fermions change value, and a neutral current interaction (NC) in which the electric charge of the interacting fermions does not change.

\subsection{Overview of the G-W-S Model}

The model of the electroweak interaction of Glashow, Weinberg and Salam $[2,3]$ is based on the gauge group $\mathrm{SU}(2) \otimes \mathrm{U}(1)$ with gauge bosons $\mathrm{W}_{\mu}^{i}, \mathrm{i}=1,2,3$ and $\mathrm{B}_{\mu}$. In the G-S-W model, the fundamental fermions (particles with spin 1/2) are divided into two groups,

- The Quarks

$$
\left(\begin{array}{l}
u \\
d
\end{array}\right),\left(\begin{array}{l}
c \\
s
\end{array}\right),\left(\begin{array}{l}
t \\
b
\end{array}\right) \text {. }
$$

- The Leptons

$$
\left(\begin{array}{c}
\nu_{e} \\
e
\end{array}\right),\left(\begin{array}{c}
\nu_{\mu} \\
\mu
\end{array}\right),\left(\begin{array}{c}
\nu_{\tau} \\
\tau
\end{array}\right) .
$$

Although the concept of chirality will be explained in section 1.3.1, it is important to note here that fermions are arranged as doublets for chiral left-handed fields and 
singlets for right handed fields. The left-handed fields, written in terms of the flavor eigenstates are:

$$
\psi^{l}=\left(\begin{array}{c}
\nu_{l} \\
l^{-}
\end{array}\right)
$$

where $\mathrm{l}=(\mathrm{e}, \mu, \tau)$ and:

$$
\psi^{q}=\left(\begin{array}{l}
u \\
d^{\prime}
\end{array}\right)
$$

where $\mathrm{u}$ describes quarks with charge $2 / 3(\mathrm{u}, \mathrm{c}, \mathrm{t})$ and $\mathrm{d}^{\prime}$ quarks with charge $1 / 3$ $\left(d^{\prime}, s^{\prime}, b^{\prime}\right)$. The mass eigenstates of quarks are not identical to flavors eigenstates, and they are connected via a mixing matrix $\mathrm{V}$

$$
d^{\prime}=\sum_{j} V d
$$

called the Cabibo-Kobayashi-Maskawa (CKM) matrix. The CKM matrix is generally written in the form :

$$
V=\left(\begin{array}{ccc}
c_{12} c_{13} & -s_{12} c_{13} & s_{13} e^{-i \delta} \\
s_{12} c_{23}+c_{12} s_{23} s_{13} e^{i \delta} & c_{12} c_{23}-s_{12} s_{23} s_{13} e^{i \delta} & -s_{23} c_{13} \\
s_{12} s_{23}-c_{12} c_{23} s_{13} e^{i \delta} & c_{12} s_{23}+s_{12} c_{23} s_{13} e^{i \delta} & c_{23} c_{13}
\end{array}\right)
$$

Here, $\mathrm{c}_{12}=\cos \theta_{12}, \mathrm{~s}_{12}=\sin \theta_{12}$, etc, and $\delta$ is the charge-parity $(\mathrm{CP})$ violating phase. The right handed fermions fields are: $\mathrm{u}_{R}, \mathrm{~d}_{R}, \mathrm{c}_{R}, \mathrm{~b}_{R}, \mathrm{t}_{R}, \mathrm{e}_{R}, \mu_{R}$ and $\tau_{R}$. It is important to note that $\nu_{R}^{l}$, with $\mathrm{l}=(\mathrm{e}, \mu, \tau)$, are not included in the G-S-W model. Introducing the fields $\mathrm{W}_{\mu}^{ \pm}$as

$$
W_{\mu}^{ \pm}=\frac{1}{\sqrt{2}}\left(W_{\mu}^{1} \pm i W_{\mu}^{2}\right)
$$


the Lagrangian of the electroweak interaction is given by:

$$
\begin{aligned}
\mathcal{L}= & \sum_{i} \bar{\psi}_{i}\left(i \gamma^{\mu} \partial_{\mu}-m_{i}-g \frac{m_{i} H}{2 m_{W}}\right) \psi_{i} \\
& -\frac{g}{2 \sqrt{2}} \sum_{i} \bar{\psi}_{i} \gamma^{\mu}\left(1-\gamma^{5}\right)\left(T^{+} W_{\mu}^{+}+T^{-} W_{\mu}^{-}\right) \psi_{i} \\
& -e \sum_{i} q_{i} \bar{\psi}_{i} \gamma^{\mu} \psi_{i} A_{\mu} \\
& -\frac{g}{2 \cos \theta_{W}} \sum_{i} \bar{\psi}_{i} \gamma^{\mu}\left(g_{V}^{i}-g_{A}^{i}\right) \psi_{i} Z_{\mu}
\end{aligned}
$$

In equation (1.5), the $4 \times 4 \gamma$-matrices are given by:

$$
\begin{gathered}
\gamma_{0}=\left(\begin{array}{cc}
1 & 0 \\
0 & -1
\end{array}\right) \\
\gamma_{i}=\left(\begin{array}{cc}
0 & \sigma_{i} \\
-\sigma_{i} & 0
\end{array}\right)
\end{gathered}
$$

where $\sigma_{i}$ are the $2 \times 2$ Pauli matrices. Here, $\theta_{W}$ is the Weinberg angle, e $=\operatorname{gsin} \theta_{W}$ is the positron electric charge, and $\mathrm{A}=\mathrm{B} \cos \theta_{W}+\mathrm{W}^{3} \sin \theta_{W}$, is the massless photon field. $\mathrm{W}^{ \pm}$and $\mathrm{Z}=-\mathrm{B} \sin \theta_{W}+\mathrm{W}^{3} \cos \theta_{W}$ are the massive charged and neutral weak boson fields respectively. $\mathrm{T}^{+}$and $\mathrm{T}^{-}$are the weak isospin raising and lowering operators. The vector and axial-vector couplings are:

$$
\begin{aligned}
& g_{V}^{i}=t_{3}(i)-2 q_{i} \sin ^{2} \theta_{W} \\
& g_{A}^{i}=t_{3}(i)
\end{aligned}
$$

where $t_{3}(i)$ is the weak isospin of fermion $i$ and is such that:

- $\mathrm{t}_{3}=1 / 2$ for $\mathrm{u}_{i}$ and $\nu_{i}$

- $\mathrm{t}_{3}=-1 / 2$ for $\mathrm{d}_{i}$ and $\mathrm{e}_{i}$

Also appearing in equation (1.5) are the mass of the fermion $\mathrm{m}_{l}$ and the charge $\mathrm{q}_{i}$, in units of the electron charge e. The second term in equation (1.5) represents 
the charge current weak interaction. The third term describes electromagnetic interactions while the last is the weak neutral current interaction.

A matrix $\mathrm{U}$, analogous the CKM matrix given in equation (1.7), allows for the neutrino flavor eigenstates $\left(\nu_{e}, \nu_{\mu}, \nu_{\tau}\right)$ to mix with three mass eigenstates $\left(\mathrm{m}_{1}, \mathrm{~m}_{2}, \mathrm{~m}_{3}\right)$. This matrix will appear in the discussion of the neutrino magnetic moment in section 1.4.2, and in the brief description of neutrino oscillation at the start of chapter 2 .

\subsection{Neutrino Properties in the G-W-S Model 1.2.1 Chirality and Helicity}

The Dirac equation which describes spin $1 / 2$ particles is given by:

$$
\left(i \gamma^{\mu} \partial_{\mu}-m_{D}\right) \psi=0
$$

Here, $\psi$ is a four-component spinor, and its chiral projections $\psi_{L}$ and $\psi_{R}$ are respectively called the left-handed an right handed composition of the spinor:

$$
\begin{aligned}
& \psi_{L}=P_{L} \psi \\
& \psi_{R}=P_{R} \psi
\end{aligned}
$$

where $\mathrm{P}_{L}$ and $\mathrm{P}_{R}$ are the projection operators given by:

$$
\begin{gathered}
P_{L}=\frac{1}{2}\left(1-\gamma_{5}\right) \\
P_{R}=\frac{1}{2}\left(1+\gamma_{5}\right) .
\end{gathered}
$$

While the spinors of all fundamental fermions can be separated into left-handed and right handed components, neutrinos in the Standard Model have only a lefthanded component. 
Helicity is the projection of a particle's spin $\vec{\sigma}$ along its direction of motion $\vec{P}$. The helicity operator is given by:

$$
H=\frac{\vec{\sigma} \cdot \vec{P}}{|\vec{P}|}
$$

The helicity is negative if the particle's spin is aligned opposite the direction of motion, and positive if it is aligned along the direction of motion. In the case of massless particles, helicity and chirality are identical, and this can be seen by rewritting equation (1.8) in mometum space for $\mathrm{m}_{D}=0$.

$$
\begin{gathered}
E \psi_{L}=\sigma_{i} p_{i} \psi_{L} \\
E \psi_{R}=-\sigma_{i} p_{i} \psi_{R}
\end{gathered}
$$

where $\mathrm{E}=\mathrm{i} \frac{\partial}{\partial t}$ and $\mathrm{p}_{i}=-\mathrm{i} \frac{\partial}{\partial x_{i}}$. From equations (1.14) and (1.15) it is deduced that left-handed particles like neutrinos have negative helicity, while right handed particles like antineutrinos have positive helicity.

If a particle is massive, then the sign of the particles helicity is frame dependent. Boosting to a frame which is moving faster than the particle will cause the helicity to flip; the sign of the momentum will change but the spin will not. Since chirality is independent of mass, its value will remain the same whether the particle is massive or not.

\subsubsection{Charge Conjugation and Parity}

While for most fermions the difference between particles and antiparticles is apparent from their electric charge, for neutrinos which are neutral, the distinction is less obvious. The operator that connects particles to their antiparticles is called charge conjugation $\mathrm{C}$. If $\psi$ is the spinor of a neutrino, the corresponding conjugated field is:

$$
\psi^{c} \equiv C \psi C^{-1}=\eta_{c} C \bar{\psi}^{T}
$$


where $\eta_{C}$ is a phase factor with $\left|\eta_{c}\right|=1$.

If $\psi$ is an eigenstate of chirality, so is $\psi^{c}$, but with an eigenvalue of opposite sign. Using the projection operators $\mathrm{P}_{L}$ and $\mathrm{P}_{R}$ defined in section 1.3.1, one gets:

$$
P_{L} \psi=\psi_{L} \rightarrow P_{L} \psi^{c}=\left(\psi^{c}\right)_{L}=\left(\psi_{R}\right)^{c}
$$

It follows from equation (1.17) that charge conjugation transforms a left-handed particle into a right handed antiparticle.

Parity on the other hand is a transformation that changes helicity. This stems from the fact that the helicity operator defined in section 1.3 .1 is a scalar product of an axial vector $(\vec{\sigma})$ and a vector $(\vec{P})$. Whereas spin preserves its orientation under mirror reflection, the direction of the momentum is reversed. In order for an interaction to conserve parity, and thus couple identically to both right and left-handed particles, it must be purely vectorial or a purely axial.

A parity transformation operation $\mathrm{P}$ is defined as:

$$
\psi(\vec{x}, t) \rightarrow P \psi(\vec{x}, t) P^{-1}=\eta_{p} \gamma_{0} \psi(-\vec{x}, t) .
$$

where $\eta_{p}$ is also a phase factor with $\left|\eta_{p}\right|=1$. Using the charge conjugate field $\psi^{c}$, it follows that:

$$
\psi^{c}=\eta_{c} C \bar{\psi}^{T} \rightarrow \eta_{c} \eta_{p}^{*} C \gamma_{0}^{T} \bar{\gamma}^{T}=-\eta_{P}^{*} \gamma_{0} \psi^{c} .
$$

\subsection{Origin of Mass}

\subsubsection{Fermion Mass}

In the G-W-S model, the notion of mass is introduced through the spontaneous breaking of the $\mathrm{SU}(2) \otimes \mathrm{U}(1)$ symmetry, known as the Higgs mechanism. To break the symmetry, this Higgs mechanism introduces a spin-zero doublet

$$
\left(\begin{array}{l}
\phi^{+} \\
\phi_{0}
\end{array}\right)
$$


and a renormalizable gauge invariant potential:

$$
V(\phi)=-\mu^{2} \phi^{+} \phi_{0}+\lambda\left(\phi^{+} \phi_{0}\right)^{2}
$$

The minimization of the potential leads to the vaccum expectation values

$$
\begin{aligned}
\left\langle\phi_{0}\right\rangle=\sqrt{\frac{\mu^{2}}{2 \lambda}} & \equiv v \\
\left\langle\phi^{+}\right\rangle & =0
\end{aligned}
$$

Fermions acquire their mass through coupling to the vaccum expectation value of the Higgs field. To conserve isospin invariance of the coupling, the Higgs doublet has to be combined with a fermion doublet and singlet. The Lagrangian describing the interaction of fermions with the Higgs field is:

$$
\mathcal{L}=g_{0} \bar{\psi}_{l}^{R}\left[\psi_{\nu}^{L}\left(\phi^{+}\right)+\psi_{l}^{L}\left(\phi_{0}\right)\right] .
$$

Replacing $\phi$ by

$$
\left(\begin{array}{c}
0 \\
\frac{v}{\sqrt{2}}
\end{array}\right)
$$

the Lagrangian becomes:

$$
\mathcal{L}=g_{0} \frac{v}{\sqrt{2}}\left(\bar{\psi}_{l}^{R} \psi_{l}^{L}+\bar{\psi}_{l}^{L} \psi_{l}^{R}\right)
$$

or simply

$$
\mathcal{L}=g_{0} \frac{v}{\sqrt{2}}(\bar{\psi} \psi)
$$

where $g_{0}$ is the Yukawa coupling constant.

To determine the significance of the symmetry breaking engendered by equation (1.24), the free Dirac Lagrangian $\mathcal{L}_{D}=\bar{\psi}\left(i \gamma^{\mu} \partial_{\mu}-m_{D}\right) \psi$ needs to be rewritten in its chiral representation:

$$
\mathcal{L}_{D}=m_{D}\left(\bar{\psi}_{L} \psi_{R}+\bar{\psi}_{R} \psi_{L}\right)
$$


Equating equation (1.23) to equation (1.26) leads to the fermions mass:

$$
m_{l}=g_{0} \frac{v}{\sqrt{2}}
$$

\subsubsection{Neutrino Mass}

Although neutrinos are fermions, the fact that they are neutral and left-handed sets them apart from the other fermions.

\section{- Dirac Mass}

The same Higgs mechanism that gives charged fermions their masses can also be used to give neutrinos mass. However, a Lagrangian analogous to equation (1.27) cannot be written because by assumption, the theory contains no right handed neutrino state $\nu_{R}$. Since a mass term cannot be written, neutrinos in the Standard Model are assumed to be massless. Right handed neutrinos can exist, but will remain undectable because they do not interact through the weak force. In this case, neutrinos will acquire their mass the same way other fermions do as described 1.3.2, and will correspond to:

$$
m_{\nu}=g_{\nu} \frac{v}{\sqrt{2}}
$$

\section{- Majorana Mass}

Another possible way for neutrinos to acquire mass is if they are their own antiparticles, i.e, Majorana particles. While the initial Dirac fermion field $\psi$ had four states: two spin states of a particle, $\psi_{R}$ and $\psi_{L}$, and two anti-particle spin states $\left(\psi^{c}\right)_{R}$ and $\left(\psi^{c}\right)_{L}$, Majorana fermion fields, which by definition are characterized by $\psi_{L}=\psi_{L}^{c}$ and $\psi_{R}=\psi_{R}^{c}$ have only two spin states. Thus, the free Lagrangian as written in equation (1.26) will include three Lorentz invariant terms and their corresponding hermitian conjugate (h.c): $\bar{\psi} \psi, \overline{\psi^{c}}{ }_{R} \psi_{L}$ and $\overline{\psi^{c}}{ }_{L} \psi_{R}$. In this case, the 
most general free Lagrangian takes the following form:

$\mathcal{L}=\bar{\psi} \gamma_{\mu} \partial^{\mu} \psi+m_{D}\left[\bar{\psi}_{L} \psi_{R}+h . c\right]+\frac{M_{L}}{2}\left[\left(\overline{\psi^{c}}\right)_{R} \psi_{L}+h . c\right]+\frac{M_{R}}{2}\left[\left(\bar{\psi}^{c}\right)_{L} \psi_{R}+h . c\right]$

where $\mathrm{m}_{D}$ is the mass term for a Dirac field while $\mathrm{M}_{L}$ and $\mathrm{M}_{R}$ are the Majorana mass terms. Using the chiral representation of a Majorana field $\psi$ :

$$
\left(\psi_{L}\right)^{c}=\frac{1+\gamma_{5}}{2} \psi^{c} \equiv\left(\psi^{c}\right)_{R}
$$

and

$$
\left(\psi_{R}\right)^{c}=\frac{1-\gamma_{5}}{2} \psi^{c} \equiv\left(\psi^{c}\right)_{L}
$$

equation(1.29) becomes:

$\mathcal{L}=\bar{\psi} \gamma_{\mu} \partial^{\mu} \psi+m_{D}\left[\bar{\psi}_{L} \psi_{R}+h . c\right]+\frac{M_{L}}{2}\left[\left(\bar{\psi}^{c}{ }_{L}\right) \psi_{L}+h . c\right]+\frac{M_{R}}{2}\left[\left(\bar{\psi}_{R}{ }_{R}\right) \psi_{R}+h . c\right]$

By defining the two Majorana fields as:

$$
\begin{aligned}
\phi_{1} & =\frac{\psi_{L}+\psi_{R}^{c}}{\sqrt{2}} \\
\phi_{2} & =\frac{\psi_{R}+\psi_{L}^{c}}{\sqrt{2}}
\end{aligned}
$$

one can write the mass terms of equation (1.32) as:

$$
\mathcal{L}_{\text {mass }}=m_{D}\left(\bar{\phi}_{1} \phi_{2}+\bar{\phi}_{2} \phi_{1}\right)+M_{L}\left(\bar{\phi}_{1} \phi_{1}\right)+M_{R}\left(\bar{\phi}_{2} \phi_{2}\right)
$$

where $\mathrm{m}_{D}$ is the Dirac mass, and $\mathrm{M}_{L}$ and $\mathrm{M}_{R}$ are the Majorana mass terms.

The introduction of a neutrino mass matrix allows the mass terms of equation (1.35) to be written as:

$$
\mathcal{L}=\frac{1}{2}\left(\bar{\phi}_{1}, \bar{\phi}_{2}\right)\left(\begin{array}{cc}
M_{L} & m_{D} \\
m_{D} & M_{R}
\end{array}\right)\left(\begin{array}{c}
\phi_{1} \\
\phi_{2}
\end{array}\right)+\text { h.c. }
$$


The diagonalization of the matrix produces two mass eigenvalues $\mathrm{m}_{1}, \mathrm{~m}_{2}$

$$
m_{1,2}=\frac{1}{2}\left[\left(M_{L}+M_{R}\right) \pm \sqrt{\left(M_{L}-M_{R}\right)^{2}+4 m_{D}^{2}}\right]
$$

and two eigenvectors $\nu^{\prime}$ and $\mathrm{N}$ which in the case of $\mathrm{M}_{L}=0{ }^{1}$ and $\mathrm{m}_{D}<<\mathrm{M}_{R}{ }^{2}$ are such that:

$$
\begin{aligned}
\nu^{\prime} & =\phi_{1}+\frac{m_{D}}{M_{R}} \phi_{2}, \quad m_{1}=-\frac{m_{D}^{2}}{M_{R}} \\
N & =\phi_{2}-\frac{m_{D}}{M_{R}} \phi_{1}, \quad m_{2}=M_{R}
\end{aligned}
$$

If neutrinos are Majorana particles, and with the assumptions of $\mathrm{M}_{L}=0$ and $\mathrm{m}_{D}<<\mathrm{M}_{R}$, the free Lagrangian of the system $L=\overline{\nu^{\prime}} \gamma_{\mu} \partial^{\mu} \nu^{\prime}+\bar{N} \gamma_{\mu} \partial^{\mu} N+m_{1} \bar{\nu}^{\prime} \nu+$ $m_{2} \bar{N} N$ indicates the presence of two neutrino states: one heavy state $\mathrm{N}$ not directly observable at low energies, and one light state $\nu^{\prime}$ corresponding to the neutrino currently observed in weak processes. This procedure, known as the seesaw mechanism, provides a natural explanation as to why the observed neutrino masses are so small.

\subsection{Electromagnetic Properties of Neutrinos}

A non-vanishing neutrino mass could show up in measurements of the electromagnetic properties of neutrinos. Although they are neutral, neutrinos can take part in electromagnetic interactions by magnetic coupling with photons in loop diagrams.

For the neutrino decay process $\nu_{i} \rightarrow \nu_{f}+\gamma$, the transition amplitude is given by:

$$
<\nu_{f}\left(p_{f}\right)\left|J_{\mu}^{e m}\right| \nu_{i}\left(p_{i}\right)>=\bar{u}\left(p_{f}\right)\left(\Gamma_{\mu}\right)_{f i} u\left(p_{i}\right)
$$

\footnotetext{
${ }^{1}$ The mass terms are obtained by introducing three scalar Higgs field, one for each of the mass terms. The expectation value of the Higgs field corresponding to $\mathrm{M}_{L}$ is set to zero because a non-zero expectation value would affect the relative strength of the $\mathrm{CC}$ and $\mathrm{NC}$ found experimentally to be approximatelly equal.

${ }^{2}$ The expectation value of the Higgs field corresponding to $\mathrm{m}_{D}$ is expected to be of the order of the mass of the quark and leptons which occur at a scale lower than the $\mathrm{SU}(2)$ symmetry breaking.
} 
where $\mid \nu_{i}>$ and $\left\langle\nu_{f}\right|$ are respectively the initial and final states of the two Dirac neutrinos $\nu_{i}$, and $\nu_{f}$ with initial momentum $\mathrm{p}_{i}$ and final momentum $\mathrm{p}_{f} \cdot\left(\Gamma_{\mu}\right)_{f i}$ is a vertex function charactrizing the decay process. Lorentz invariance in the electromagnetic interaction implies that the vertex function can have ten types of couplings: five vector couplings, and five axial couplings. The vector couplings are $q_{\mu}, \gamma_{\mu}, P_{\mu}, \sigma_{\mu \nu} \mathrm{q}^{\nu}, \sigma_{\mu \nu} \mathrm{P}^{\nu}$, where $q_{\mu}=p_{f}-p_{i}, \mathrm{P}=p_{f}+p_{i}$ and $\sigma_{\mu \nu}=\frac{1}{2}\left[\gamma_{\mu}, \gamma_{\nu}\right]$. The axial vectors are obtained by the inclusion of a $\gamma_{5}$ factor.

Requiring that the couplings obey the Dirac equation in equation (1.8) and the electromagnetic current be conserved, $q^{\mu} J_{\mu}^{e m}=0$, reduces the number of independent couplings, such that the most general electromagnetic current element between two neutrino states is given by:

$$
\begin{aligned}
<\nu_{f}\left(p_{f}\right)\left|J_{\mu}^{e m}\right| \nu_{i}\left(p_{i}\right)>= & \bar{u}\left(p_{i}\right)\left[V_{2} \gamma_{\mu}+i V_{3} \sigma_{\mu \nu} q^{\nu}\right] u\left(p_{i}\right) \\
& +\bar{u}\left(p_{i}\right)\left[A_{2}\left(q^{2} \gamma_{\mu}-q_{\mu} q_{\nu} \gamma^{\nu}\right) \gamma_{5}-i A_{3} \sigma_{\mu \nu} q^{\nu} \gamma_{5}\right] u\left(p_{i}\right)
\end{aligned}
$$

$V_{2}$ and $V_{3}$ are respectively called the charge moment form factor $\mathrm{f}_{Q}\left(\mathrm{q}^{2}\right)$, and the magnetic dipole form factor $\mathrm{f}_{M}\left(\mathrm{q}^{2}\right) . A_{2}$ and $A_{3}$ are respectively the anapole form factor $\mathrm{f}_{A}\left(\mathrm{q}^{2}\right)$, and the electric dipole form factor $\mathrm{f}_{E}\left(\mathrm{q}^{2}\right)$.

If neutrinos are Dirac particles, the assumption of CP invariance and the hermicity of the electromagnetic current operator $J_{\mu}^{e m}$ leads to $\mathrm{A}_{3}=0$. On the other hand, for Majorana neutrinos, all the terms but the anapole expression $f_{A}\left(q^{2}\right)$ vanish because of the self conjugate property.

\subsubsection{Charge Radius}

Even though neutrinos carry a zero net charge, under arbitrary quantum fluctuation they can have a nonzero charge radius which is manifested as a radiative correction of the weak neutral-current vector coupling. The size of the correction 
is given by:

$$
\delta=\frac{\sqrt{2} \pi \alpha}{3 G_{F}}<r^{2}>
$$

By definition, the charge radius is the differential of the electric dipole form factor for $\mathrm{q}^{2}=0$.

$$
<r^{2}>=\left.6 \frac{\partial f_{E}\left(q^{2}\right)}{\partial q^{2}}\right|_{q^{2}=0}
$$

The mean square radius can be measured in the neutrino-electron scattering, and the current limits set by various experiments are:

$$
\begin{aligned}
& \text { - }<r^{2}>\left(\nu_{e}\right)<4.88 \times 10^{-32} \mathrm{~cm}^{2}(\text { LAMPF [4] ) } \\
& \text { - }<r^{2}>\left(\nu_{\mu}\right)<1.0 \times 10^{-32} \mathrm{~cm}^{2}(\mathrm{CHARM}[5]) \\
& \text { - }<r^{2}>\left(\nu_{\mu}\right)<6.0 \times 10^{-32} \mathrm{~cm}^{2}(\mathrm{CHARM} \text { II [6]) } \\
& \text { - }<r^{2}>\left(\nu_{\mu}\right)<2.4 \times 10^{-32} \mathrm{~cm}^{2}(\mathrm{E} 734[7])
\end{aligned}
$$

\subsubsection{Neutrino Magnetic Moment}

The neutrino magnetic moment for neutrinos produced at the source as $\nu_{\ell},(\ell=$ $e, \mu, \tau)$, with energy $\mathrm{E}_{\nu}$ and travelling a distance $\mathrm{L}$ can be described as [8]:

$$
\mu_{\ell}^{2}=\sum_{j}\left|\sum_{k} U_{\ell k} e^{-i E_{\nu} L} \mu_{j k}\right|^{2}
$$

where $\mathrm{U}_{l k}$ is the neutrino mixing matrix, and $\mu_{j k}$ is related to the electric $\mathrm{f}_{E}\left(\mathrm{q}^{2}\right)$ and magnetic $\mathrm{f}_{M}\left(\mathrm{q}^{2}\right)$ dipole moments which couple together the neutrino mass eigenstates $\nu_{j}$ and $\nu_{k}$. The dipole moments $\mathrm{f}_{E}\left(\mathrm{q}^{2}\right)$ and $\mathrm{f}_{M}\left(\mathrm{q}^{2}\right)$ are defined in equation 1.42 .

The observable $\mu_{\nu}$ is therefore an effective magnetic moment, and the $\nu_{\mu}$ e electromagnetic scattering cross section resulting from the coupling of a neutrino with 
a photon can be written as:

$$
\left(\frac{d \sigma}{d T}\right)^{E M}=\frac{\pi \alpha^{2}}{m_{e}} \mu_{\nu}^{2}\left[\frac{1}{T}-\frac{T}{E_{\nu}}\right]
$$

where $\mathrm{T}$ is the kinetic energy of the recoil electron.

As will be described in chapters 2 and 3, for MiniBooNE the neutrino beam at the source is over $99 \% \nu_{\mu} \mathrm{s}$, and thus in chapter 5 of this thesis, the interaction $\nu_{\mu} e \rightarrow \nu_{\mu} e$ will be used to set a limit on the effective magentic moment of the muon neutrino.

Given a specific model, $\mu_{\nu}$ can be calculated from first principles. For the minimally extended Standard Model with massive Dirac neutrinos the magnetic moment is written as:

$$
\mu_{\nu}=\frac{3 e G_{F}}{8 \pi^{2} \sqrt{2}} m_{\nu}
$$

The presence of the neutrino mass term is necessary to flip the neutrino helicity and induce a magnetic moment. Because of the mass dependence, the magnetic moment of Dirac neutrinos is expected to be small [9], on the order of $\mu_{\nu_{i}}=$ $3.20 \times 10^{-19} \mu_{B}\left(\frac{m_{\nu_{i}}}{1 e V}\right),\left(\mu_{B}=\frac{e}{2 m_{e}}\right.$ is the Bohr magneton $)$ which is far too small to have any observable consequences. However, additional physics beyond the Standard Model, such as Majorana neutrinos or right handed weak currents [10], can significantly enhance $\mu_{\nu}$ to experimentally relevant values.

Chapter 5 of this thesis will be devoted to the search for a neutrino magnetic moment using data from the MiniBooNE experiment. The analysis will be looking for an excess of low energy $\nu_{\mu}$ e events above the Standard Model prediction from which we will derive an upper limit on the neutrino magnetic moment. Current neutrino magnetic moment limits are obtained from astrophysics bounds as well as from laboratory experiments. 


\section{- Astrophysics Bounds}

The magnetic moment limits from astrophysics heavily rely on the consequences of chirality flip of neutrino states in the astrophysical medium. The most popular is the spin flavor precession mechanism that was once used as a possible explanation of the solar neutrino deficit [11]. The solar neutrino $\nu_{e}$ would interact with the solar magnetic field to become a neutrino of different flavor $\nu_{j}$, with $\mathrm{j} \neq \mathrm{e}$. The typical predicted size is $\mu_{\nu}$ (astrophysics) $<10^{-10}-10^{-12} \mu_{B}$. This scenario, although compatible with the solar neutrino data has been ruled out by the KamLAND experiment [12] which favors the Large Mixing Angle (LMA) parameter space as the solution of the solar neutrino deficit. Other limits come from the observations on Big Bang Nucleosynthesis [13], stellar cooling via plasmon decay [14] and the cooling of supernova 1987a $[15,16]$.

\section{- Experimental Results}

Direct measurement of neutrino magnetic moment limits come from various neutrino sources that include solar, accelerator and reactor. The experiments require an understanding of the neutrino energy spectra and the neutrino composition at the detector. These experiments typically study neutrino-electron scattering $\nu_{l}+e \rightarrow \nu_{l}+e$, and as mentioned in section 1.4.2 the signature is an excess of events above the Standard Model prediction, which exhibit the characteristic 1/T spectral dependence.

A limit on the neutrino magnetic moment from solar neutrinos is provided by the Super-Kamiokande (SK) experiment [17] which set the limit by analyzing the spectral distortion of the recoil electron from $\nu+e \rightarrow \nu+e$ scattering. At the $90 \%$

Confidence Level (C.L.), SK found $\mu_{\nu}$ (solar $)<1.1 \times 10^{-10} \mu_{B}$ 
The best limit from accelerator experiments comes from the LSND experiment. LSND measured electron events from a beam with a known mixture of $\nu_{\mu}, \overline{\nu_{\mu}}, \nu_{e}$ fluxes and spectral composition. At the $90 \%$ C.L., limits of $\mu_{\nu}\left(\nu_{e}\right)<1.1 \times 10^{-9} \mu_{B}$ and $\mu_{\nu}\left(\nu_{\mu}\right)<6.8 \times 10^{-10} \mu_{B}$ were derived [18] .

Neutrino-electron scattering was first observed for reactor neutrinos in the pioneering experiment at Savannah River led by F. Reines. An analysis of the data indicated a small excess of elastic scattering events from which a limit of $\mu_{\nu}\left(\overline{\nu_{e}}\right)<$ $2-4 \times 10^{-10} \mu_{B}$ was derived [19]. However, the best limit from a reactor neutrino experiment is given by the TEXONO experiment that set a limiting value of $\mu_{\nu}\left(\overline{\nu_{e}}\right)<0.74 \times 10^{-10} \mu_{B}$ at the $90 \%$ C.L $[20]$.

\subsection{Neutrino-Nucleon Scattering}

The interaction of neutrinos with nucleons can proceed via the charged current $(\mathrm{CC})$, involving the exchange of a charged boson, or neutral current (NC) where a neutral boson is exchanged. The largest contributions to the neutrino-nucleon scattering cross section in the MiniBooNE energy region arise from the charged current quasi-elastic (CCQE) scattering, and single and multiple pion production. This thesis will focus on single pion production, but details regarding the other interactions can be found elsewhere [21, 22].

\subsubsection{Resonant Production}

Single pion production in neutrino-nucleon interactions arises primarily from the excitation of the nucleon into a resonant state, $\nu_{\mu}+N \rightarrow \mu^{-}+N^{\star}$, and the subsequent decay of the resonant state. In the few $\mathrm{GeV}$ range, the interaction is dominated by the the $\Delta(1232)$ resonance, although contributions from resonances such as $\mathrm{N}(1440)$ and $\mathrm{N}(1535)$ are non-negligible. Typical reactions in which resonance states like the $\Delta(1232)$ contribute to charged current processes (CC) are: 
- $\nu_{\mu} \mathrm{p} \rightarrow \mu^{-} \mathrm{p} \pi^{+}$

- $\nu_{\mu} \mathrm{n} \rightarrow \mu^{-} \mathrm{n} \pi^{+}$

- $\nu_{\mu} \mathrm{n} \rightarrow \mu^{-} \mathrm{p} \pi^{0}$

The correponding neutral current processes (NC) are:

- $\nu_{\mu} \mathrm{p} \rightarrow \nu_{\mu} \mathrm{p} \pi^{0}$

- $\nu_{\mu} \mathrm{n} \rightarrow \nu_{\mu} \mathrm{n} \pi^{0}$

- $\nu_{\mu} \mathrm{p} \rightarrow \nu_{\mu} \mathrm{n} \pi^{+}$

- $\nu_{\mu} \mathrm{n} \rightarrow \nu_{\mu} \mathrm{p} \pi^{-}$

\subsubsection{Coherent Production}

Other than resonance processes, charged current single pions can be produced when neutrinos scatter off the entire nucleus with a small energy transfer to produce a pion:

$\nu_{\mu}+A \rightarrow \mu^{-}+A+\pi^{+}$. Coherent processes will be briefly discussed in chapter 3 .

\subsubsection{Motivation of the $\mathrm{CC} \pi^{+}$Cross Section Study}

The motivation behind the study of the $\mathrm{CC} \pi^{+}$interaction cross section can be found in neutrino oscillation experiments because most of these experiments use the $\mu^{-}$kinematics from CCQE processes $\left(\nu_{\mu}+n \rightarrow \mu^{-}+\mathrm{p}\right)$ to reconstruct the neutrino energy. A serious background for such experiments can come from $\mathrm{CC} \pi^{+}$ processes where the $\pi^{+}$is undetected and where the $\mu^{-}$can be misidentified as a $\mu^{-}$ from CCQE. The main reason the $\pi^{+}$can go undetected is related to nuclear effects in neutrino-nucleon interactions. These nuclear effects, which will be discussed in chapter 3, include the Pauli exclusion principle, pion charge exchange and pion absorption. 
Although charged current single pion production $\left(\mathrm{CC} \pi^{+}\right)$has been studied since the early 1960 s $[23,24]$ the interaction cross section is not well understood in the neutrino energy region near $1 \mathrm{GeV}$, which is the energy region of interest for many neutrino oscillation experiments, including MiniBooNE. Also, many of the data that do exist come from experiments that used hydrogen and deuterium for nuclear targets. Current neutrino experiments use complex nuclear targets like carbon or oxygen, making a compelling case for MiniBooNE to study the $\mathrm{CC} \pi^{+}$ cross section in order to understand nuclear effects on carbon to make the necessary corrections to the CCQE oscillation samples.

\subsubsection{Resonant Charged Current Single $\pi^{+}$Cross Section}

The general form of the matrix element for resonant $\left(\nu_{\mu}+N \rightarrow \mu^{-}+N^{\star}\right)$ production where a virtual boson is exchanged can be written as:

$$
M=\frac{G_{F} \cos \theta}{\sqrt{2}} J_{l}^{\mu} J_{\mu}^{h}
$$

where $\mathrm{J}_{l}^{\mu}$ is the leptonic current given by:

$$
J_{l}^{\mu}=\left[\bar{u}_{l} \gamma^{\mu}\left(1-\gamma_{5}\right) u_{\nu}\right]
$$

and $\mathrm{J}_{h}^{\mu}$ is the hadronic current which is written in terms of form factors.

The leptonic current is often written in terms of the polarization states of the intermediate vector boson: left-handed $e_{L}^{\mu}$, right-handed $e_{R}^{\mu}$ and scalar $e_{S}^{\mu}$. In the rest frame of the resonance, the leptonic current is:

$$
\bar{u}_{l} \gamma^{\mu}\left(1-\gamma_{5}\right) u_{\nu}=-2 \sqrt{2} E_{\nu} \sqrt{\frac{Q^{2}}{|\mathbf{q}|^{2}}}\left(u \cdot e_{L}^{\mu}-v \cdot e_{R}^{\mu}+\sqrt{2 u v} \cdot e_{S}^{\mu}\right)
$$

where $\mathrm{Q}^{2}=-\mathrm{q}^{2}$ is the four-momentum transfer, $\mathbf{q}$ is the momentum in the lab frame, $\mathrm{u}=\left(E_{\nu}+E^{\prime}+Q\right) / 2 E_{\nu}$ and $\mathrm{v}=\left(E_{\nu}+E^{\prime}-Q\right) / 2 E_{\nu}$. Note that $\mathrm{u}$ and $\mathrm{v}$ are expressed in terms of the neutrino initial $\left(\mathrm{E}_{\nu}\right)$ and final energy $\left(\mathrm{E}^{\prime}\right)$ which is the 
lepton (in the case of charged current) or neutrino (in the case of neutral current) energy.

The full matrix element can be written as:

$$
M=\frac{G_{F} \cos \theta}{\sqrt{2}}\left[\bar{u}_{l} \gamma^{\mu}\left(1-\gamma_{5}\right) u_{\nu}\right]<N^{*}\left|J_{\mu}^{h}\right| N>
$$

leading to the full differential cross section for the production of a given resonance:

$$
\frac{d \sigma}{d q^{2} d W}=\frac{1}{32 \pi m_{N} E_{\nu}^{2}} \frac{1}{2} \sum_{\text {spins }}\left|M\left(\nu_{\mu}+N \rightarrow \nu_{\mu}+N^{\star}\right)\right|^{2} \frac{1}{2 \pi} \frac{\Gamma}{\left(W-m_{N}\right)^{2}+\Gamma^{2} / 4}(1
$$

where $\mathrm{W}$ is the invariant mass and is equal to $\left(p_{N}+\mathrm{p}_{\pi}\right)^{2}, \mathrm{~m}_{N}$ is the nucleon mass. The factor after the squared matrix element is a Breit-Wigner function accounting for the finite width of the resonance.

The cross section for the production of each final state is determined from summing the contributions from each resonance, using appropriate factors determined by isospin Clebsch-Gordon coefficients.

\subsection{Plan of the Thesis}

Part I of this thesis starts in chapter 2 with an overview of the MiniBooNE experiment, and continues in chapter 3 with a discussion of the Monte Carlo simulation. Chapter 4 provides the details of the reconstruction algorithm and the particle identification.

Part II of the thesis presents the different analyses and their results. Chapter 5 discusses the analysis of the process $\nu_{\mu}+e \rightarrow \nu_{\mu}+e$ for low energy elastic scattered electrons, and the subsequent measurement of the neutrino magnetic moment. In chapter 6 , the study of the elastic scattering is extended to the higher energy regime. In Chapter 7 we break with elastic scattering and discuss the cross section measurement of single pion production. In chapter 8 we conclude. 


\section{Chapter 2}

\section{MiniBooNE Experiment}

The Mini Booster Neutrino Experiment (MiniBooNE) is motivated by the observations of the Liquid Scintillator Neutrino Detector (LSND) which reported in 1997 that it had observed an excess of $\overline{\nu_{e}}$ in its $\overline{\nu_{\mu}}$ neutrino beam $[25,26]$. Neutrino oscillation experiments typically report their results in terms of two parameters: $\Delta m^{2}$, the difference of the squares of two mass eigenvalues and $\sin ^{2} 2 \theta$ which describes the mixing between the mass and flavor eigenstates. The excess reported by LSND is consistent with $0.2<\Delta m^{2}<10 \mathrm{eV}^{2}$ and $0.003<\sin ^{2} 2 \theta<0.03$, and was interpreted as $\overline{\nu_{\mu}} \rightarrow \overline{\nu_{e}}$ oscillation, with a probability of $\sim 0.3 \%$. Part of the region of parameter space $\left(\Delta m^{2}, \sin ^{2} 2 \theta\right)$ where LSND reported a possible oscillation signal was ruled out by other experiments [27, 28]. Although the Karlsruhe Rutherford Medium Energy Neutrino Experiment (KARMEN) observed no evidence for neutrino oscillation [29], a joint analysis [30] showed compatibility at the $64 \%$ CL. Hence, MiniBooNE was designed with the specific goal of resolving the unconfirmed LSND signal. In April 2007, MiniBooNE presented its first oscillation result, and reported that it observed no evidence of $\nu_{\mu} \rightarrow \nu_{e}$ oscillation within a two neutrino appearance-only model [31].

With over 1.7 million neutrino interactions collected from 2003 to 2005, MiniBooNE can also address non-oscillation physics. This thesis will focus on a search for a neutrino magnetic moment and the measurement of the cross section of charged current single pion production. 


\subsection{Neutrino Beam}

The neutrino beam at MiniBooNE is created by directing $8 \mathrm{GeV}$ protons from the Fermilab Booster onto a beryllium target. Proton interactions in the target material produce a secondary beam of mesons that subsequently decay to produce a neutrino beam with mean energy $\sim 0.8 \mathrm{GeV}$. A schematic representation of the experiment is shown in Figure 2.1, and the different elements of the figure are the subjects of the following sections.
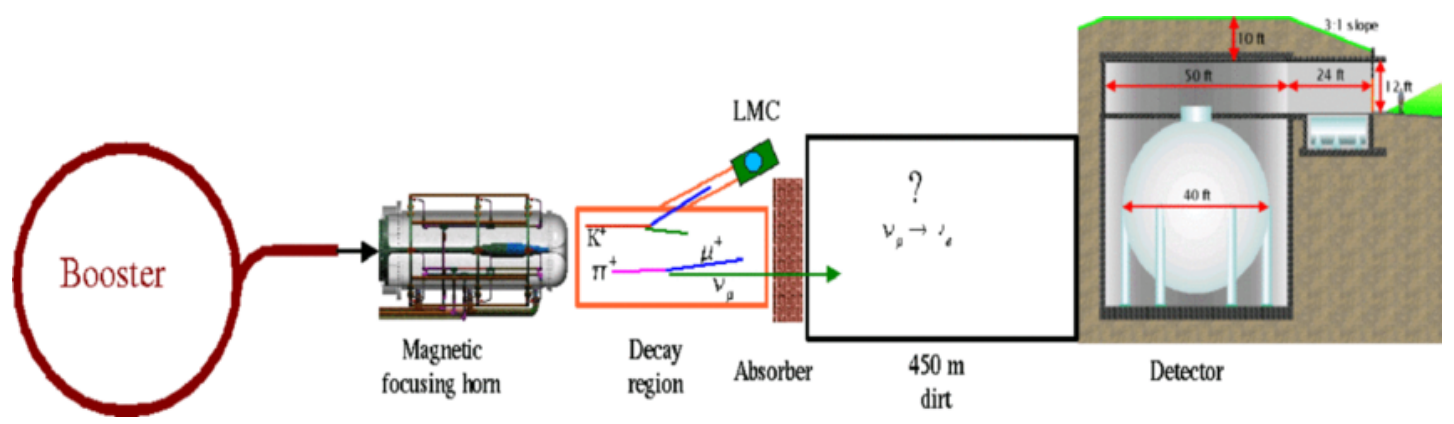

FIGURE 2.1. A schematic representation of the MiniBooNE beamline and detector

\subsubsection{Proton Source}

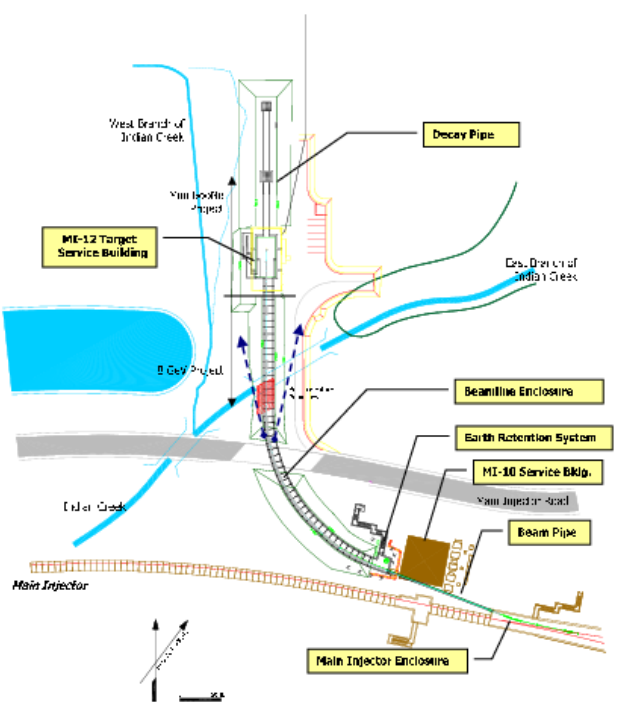

FIGURE 2.2. Booster fixed target facility with the $8 \mathrm{GeV}$ beamline. 
Figure 2.1.1 shows how the proton beam from the Booster is extracted into the $8 \mathrm{GeV}$ beamline. Typically, $\sim 4 \times 10^{12}$ protons are delivered in a $1.6 \mu$ sec-long pulse at a rate of $\sim 4 \mathrm{~Hz}$. The number of protons in each spill is measured by two toroids before they impinge on a $71 \mathrm{~cm}$-long beryllium target located inside a magnetic focusing horn.

\subsubsection{Horn and Target}

The MiniBooNE horn shown in Figure 2.1.2 was designed by Bartoszek Engineering. The horn provides a toroidal magnetic field that focuses positively charged mesons toward the decay region. Each time the protons arrived at the target, the horn is pulsed with $174 \mathrm{kA}$ of current, with each pulse lasting $143 \mu \mathrm{sec}$ and producing a magnetic field of $\sim 1 \mathrm{~T}$. Current flows along an inner conductor whose radius varies from $2.2 \mathrm{~cm}$ to $6.5 \mathrm{~cm}$, and back along an outer conductor (radius 30 $\mathrm{cm}$, length $185.4 \mathrm{~cm}$ ) to produce a toroidal magnetic field that is contained in the volume between the two coaxial conductors.

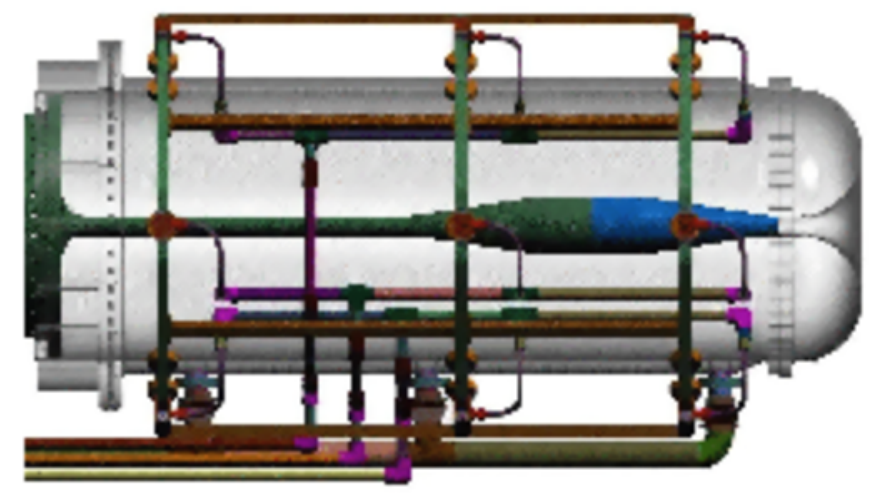

FIGURE 2.3. A schematic representation of the MiniBooNE horn. The beryllium target is located inside the focusing magnet (green). 
Given that the interaction length, $\lambda_{I}$, for protons in berrylium is $41.8 \mathrm{~cm}$ and the target is $71 \mathrm{~cm}$ long, the fraction of the beam that interacts with the target is given by $1-\mathrm{e}^{-71 / \lambda_{I}}$ and is $82 \%$. The target is made up of seven cylindrical beryllium slugs rather than one solid piece in order to minimize forces due to asymmetric heat loads from the proton beam line. Mesons produced by the p-Be interactions and focused by the horn pass through a collimator with a $30 \mathrm{~cm}$ radius aperture, located $\sim 2 \mathrm{~m}$ downstream from the end of the horn. The collimator is used to absorb secondary particles which are destined to miss the decay pipe.

\subsubsection{Decay Region}

Mesons that pass through the collimator enter a $\sim 50$ meter long, $90 \mathrm{~cm}$ radius decay pipe. The fraction of mesons that decay over a distance of $50 \mathrm{~m}$ is given by 1- $\exp [-50.0 / \gamma \beta c \tau]$ where $\gamma$ and $\beta$ are the usual relativistic parameters, $\mathrm{c}$ is the speed of light and $\tau$ the meson lifetime. The dominant decay modes for MiniBooNE are $\pi^{+} \rightarrow \mu^{+} \nu_{\mu}$ and $\mathrm{K}^{+} \rightarrow \mu^{+} \nu_{\mu}$ which produce over $90 \%$ of the neutrino beam. The lifetimes of the $\pi^{+}$and $\mathrm{K}^{+}$are repectively 26.03 and 12.37 ns. The $\nu_{e}$ component of the beam arising from $\mathrm{K}^{+} \rightarrow \pi^{0} e^{+} \nu_{e}, \mu^{+} \rightarrow \mathrm{e}^{+} \bar{\nu}_{\mu} \nu_{e} \mathrm{~K}_{L}^{0} \rightarrow \pi^{0} e^{+} \nu_{e}$, and $\mathrm{K}_{L}^{0} \rightarrow$ $\pi^{+} e^{-} \bar{\nu}_{e}$ is $0.6 \%$. The neutrino flux, shown in figure 3.3, has an average neutrino energy of $0.8 \mathrm{GeV}$.

Since muons have a relatively long lifetime (2197 ns), most of them reach the end of the decay pipe where they are stopped by a steel and concrete absorber. The $\nu_{e} \mathrm{~s}$ arising from muon and kaon decays are an intrinsic background for the oscillation analysis. The $\nu_{e} \mathrm{~s}$ from muon decay are constrained by measuring the $\nu_{\mu} \mathrm{s}$ from $\pi$ decay in the MiniBooNE detector. To help constrain the $\nu_{e}$ background from kaon decay, a kaon monitor was installed $7^{\circ}$ off-axis from the decay pipe. 


\subsubsection{Detector}

The MiniBooNE neutrino detector, shown in figure 2.1.4, is a spherical steel tank of radius $610 \mathrm{~cm}$, located beneath $3 \mathrm{~m}$ of soil for cosmic ray shielding. The detector is divided into an inner sphere of radius $5.75 \mathrm{~m}$, refered to as the signal region, and an outer shell with outer radius $6.1 \mathrm{~m}$. The two regions are separated by an optical barrier, but share oil circulation. The inside of the optical barrier is instrumented with 1280 radially inward-facing photo-multiplier tubes (PMTs) which view the detector fiducial volume. The outside of the optical barrier supports 240 pair-mounted PMTs, which view the outer shell of oil. This outer shell region is used to check the containment of neutrino events and veto incoming particles, typically cosmic rays. The PMTs are designed to detect light produced when charged particles traverse the oil. They are sensitive to $\check{C}$ erenkov radiation from relativistic particles moving faster than the speed of light in the oil, and also to isotropic light emitted through the natural scintillation of the oil. Both $\check{C}$ erenkov radiation and scintillation light are discussed in chapter 3. Of the 1520 PMTs in the MiniBooNE detector, 1197 are inherited from LSND and the other 323 were purchased from Hamamatsu [32]. The LSND PMTs are 9-stage, Hamamatsu model R1408 PMTs while the new PMTs are 10-stage, Hamamatsu model R5912 PMTs. The technical specifications for both of these types of photomultiplier tubes may be found in [32]. Both types of PMTs are 8 inches in diameter as shown in figure 2.1.4, and have about $20 \%$ quantum efficiency for emitting photoelectrons for incident photons with wavelength $\lambda \sim 400 \mathrm{~nm}$ [32]. The PMTs are operated with $\sim+2000$ $\mathrm{V}$ on the dynode chain, resulting in an average gain of $10^{8}$. The intrinsic time resolution of the PMTs is $\sim 1 \mathrm{~ns}$, and the intrinsic charge resolution is $\sim 15 \%$ at 1 photoelectron (p.e) [32]. The charge resolution is further smeared by the signal 


\section{MiniBooNE Detector}

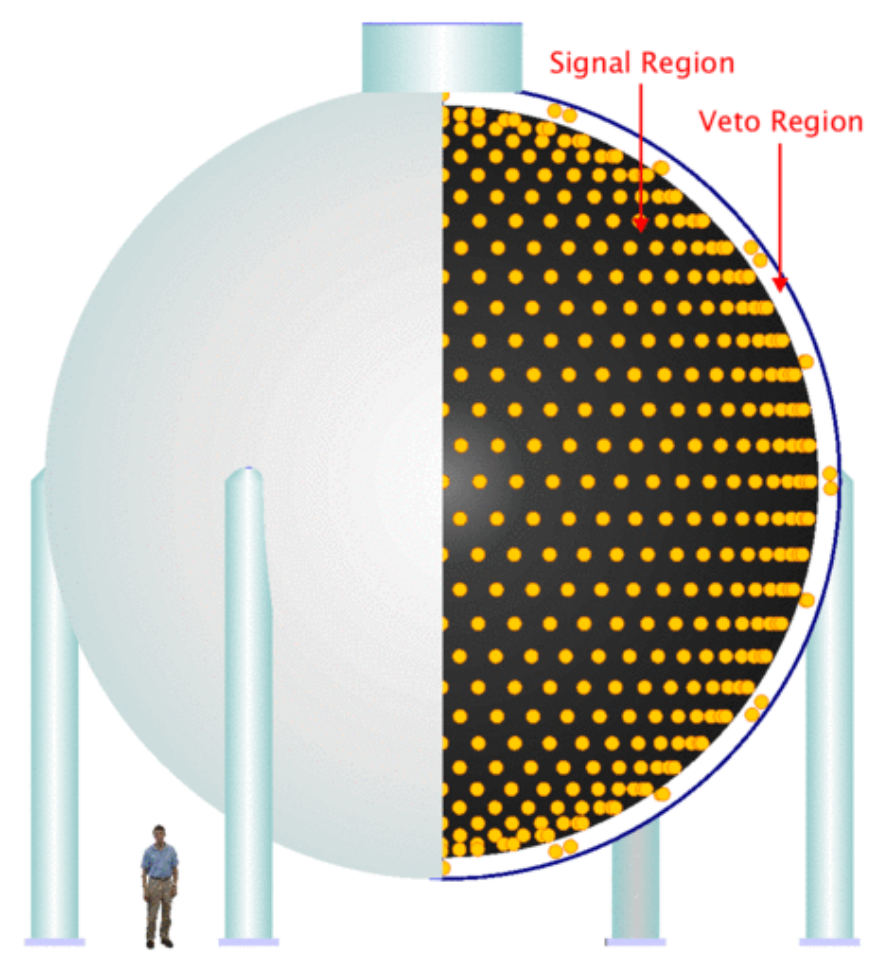

FIGURE 2.4. MiniBooNE detector showing inner volume and outer veto shell

processing electronics. However, the dominant contribution to both the charge and time resolutions arises from the intrinsic PMT properties.

The tank is filled with 800 tons of mineral oil $\left(\mathrm{CH}_{2}\right)$ that serves both as the target for neutrino interactions and the medium that produces and propagates light to its detection point on the PMT surface. Understanding the oil properties is instrumental in the detector simulation as discussed in chapter 3 .

\subsection{Data Acquisition}

MiniBooNE uses an upgraded version of the LSND PMT electronics [33], which has one channel per PMT. The voltage each PMT receives is regulated by a step-down 


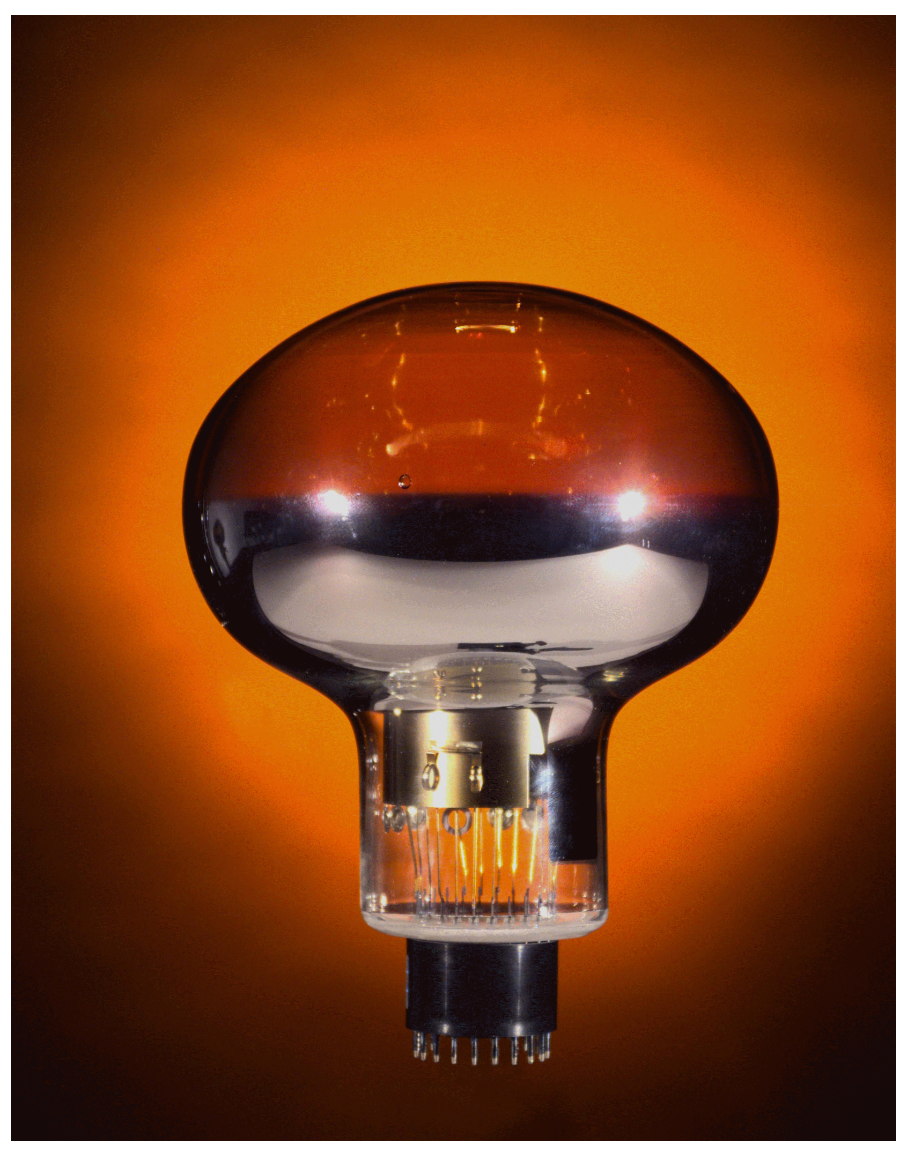

FIGURE 2.5. A picture of a 8-inch PMT used in the MiniBooNE experiment

resistor located on a preamplifier card in the electronics area above the detector. PMT signals are amplified and integrated. The time $(\mathrm{T})$ and charge $(\mathrm{Q})$ signals defined below, from each PMT are digitized by 8-bit ADCs every 100 ns, where we define 100 ns as a clock tick. The $\mathrm{T}$ and $\mathrm{Q}$ values are stored in a circular FIFO buffer that we will also describe below. A schematic representation of the digitization for one channel is shown in figure 2.2. The preamplified PMT signal, Vpmt, is integrated in a capacitive circuit located on a charge/time board (QT board), generating a second signal, Vq. If Vpmt crosses a threshold corresponding to approximately 0.25 photoelectrons, a discriminator is fired, starting a linear time ramp (Vt). The time signal is also digitized to allow a precise determination of the time at which the PMT signal crossed the threshold. This is necessary since 


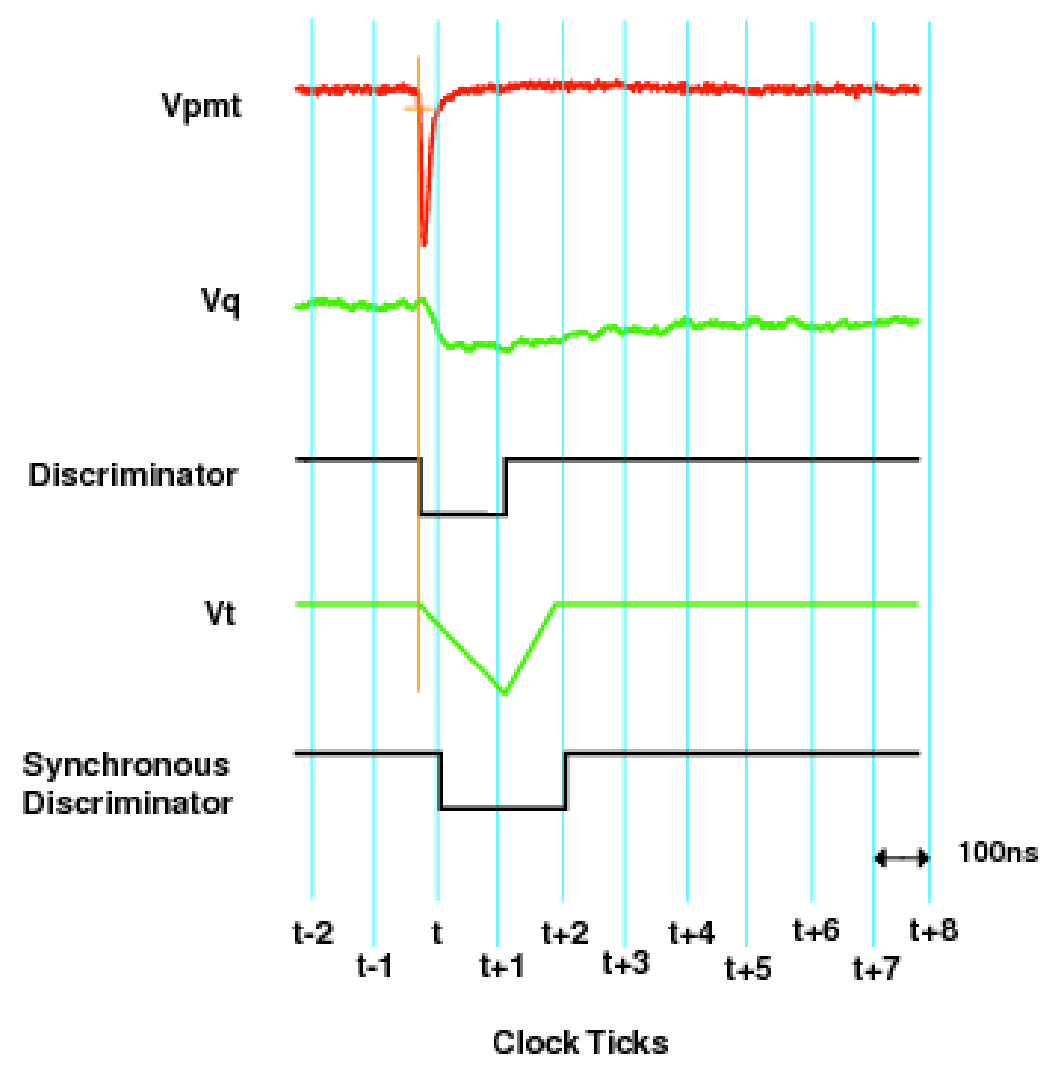

FIGURE 2.6. PMT charge and time signals. The anode pulse is given by $\mathrm{V}_{p m t}$ and the integrated charge by $\mathrm{V}_{q}$. The Discrimator digial pulse and the $\mathrm{V}_{t}$ time ramp are started when the anode pulse crosses a preset threshold.

MiniBooNE event reconstruction requires a time accuracy much better than that provided by the 100 ns clock cycles.

The entire QT system consists of 12 VME crates which are widely used in many commercial and scientific applications to control the flow of data to and from computers. Each crate contains 16 cards with 8 channels per card, resulting in 1536 available channels to serve the 1520 PMTs in the detector. There are 10 crates for tank PMTs and the two remaining crates host the veto PMT channels. Figure 2.2 shows a diagram of the circuitry for a single PMT channel. Each channel digitizes the charge and time information for a particular PMT and stores the information in a circular buffer (dual-port sRAM) at an address determined by the 11 bits of 


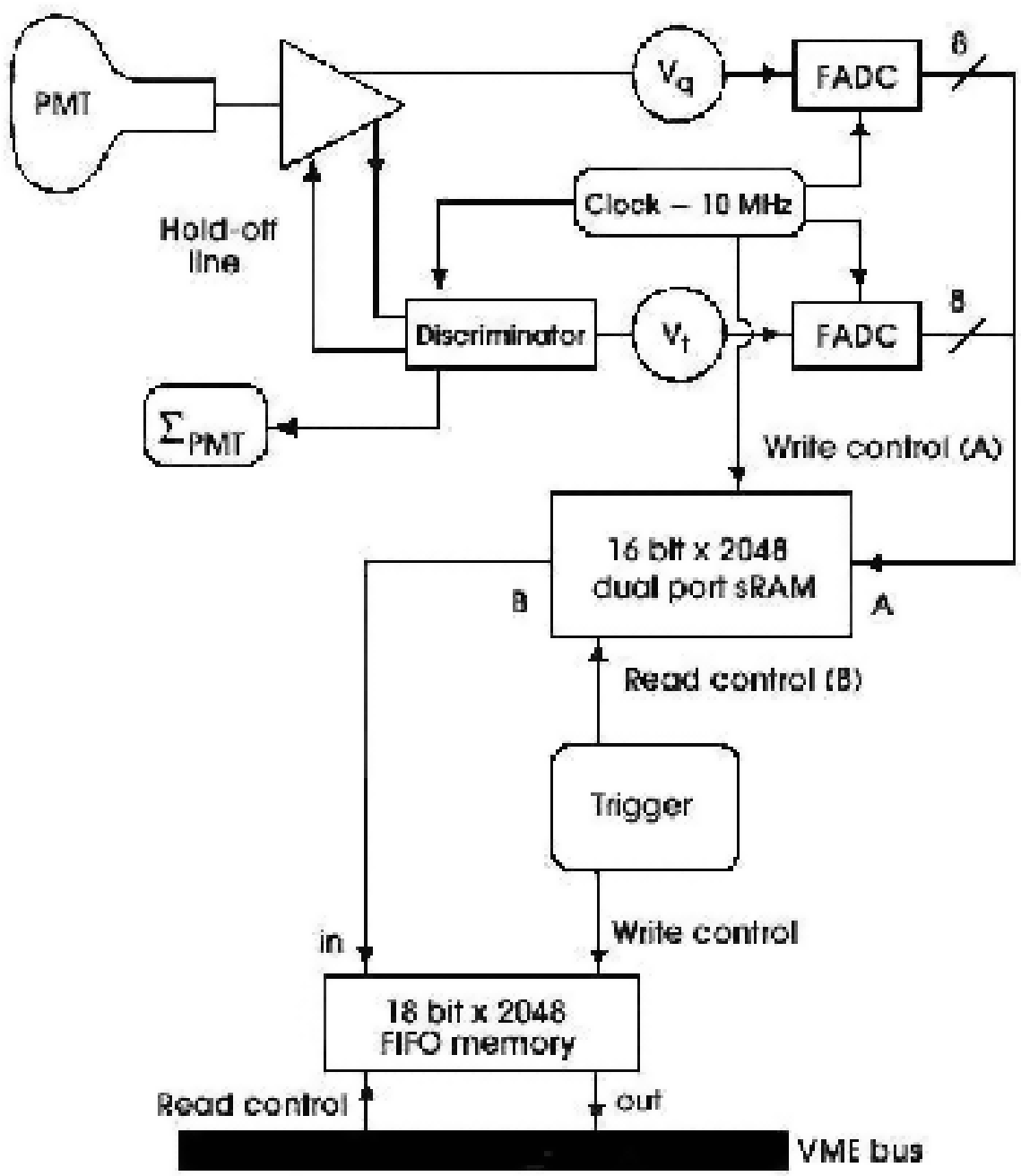

FIGURE 2.7. Schematic diagram of data acquisition electronics for a single PMT channel.

the $10 \mathrm{MHz}$ system clock. This address is known as the time-stamp address (TSA). The data are continuously digitized and written to the circular buffer, which wraps around every $204.8 \mu \mathrm{sec}$. Data are read from the circular buffer for TSAs that are requested by the trigger. If the trigger decision is too slow $(>204.8 \mu \mathrm{sec})$ in asking for data from a particular set of TSAs, the circular buffer will be overwritten, and the data of interest will be lost. A latency filter is applied to all analyzed data to reject events for which this occurs. The fraction of beam events rejected by this filter is typically negligible. 


\subsection{Trigger Sytem}

The triggering system examines bit patterns as discssed below, to determine whether or not a particular DAQ time window (set of TSAs) should be read out from the circular buffer. Each VME crate contains a single-board computer (SBC) in addition to its 16 QT cards. When trigger conditions are met, the data from the TSAs of interest are retrieved from the circular buffers in each VME crate by the $\mathrm{SBC}$ via the VME bus and shipped to the DAQ host computer where they are assembled and written to disk.

Each QT crate also contains a PMT sum card which counts the number of channels that caused the discriminator to fire in the last 2 clock cycles (200 ns). This information is routed to the trigger crate, which contains main and veto sum cards. These cards take the sum of sums for main and veto crates separately to give an overall number of PMT signals in the main and veto regions of the detector.

The MiniBooNE trigger hardware has four external inputs for NIM signals and seven trigger bit settings which are used to decide whether to read out the detector on a given clock tick. The trigger table is constructed in software using combinations of the hardware triggers and trigger activity timing information. The trigger hardware external inputs are:

- Beam trigger (E1): the beam-on-target trigger.

- Strobe trigger (E2): a $2.01 \mathrm{~Hz}$ random strobe, triggered by a pulser.

- Calibration trigger (E3): the calibration trigger, which has a different NIM pulse length depending on whether it is a laser, cube, or tracker calibration event.

- Hardware 'OR' (E4): a NIM hardware OR of the previous three conditions. 
The seven internal hardware trigger bits (DET1-DET5, VETO1, and VETO2) are asserted if the main (veto) sum card indicates the presence of a minimum number of PMT signals in the main (veto) detector region. Table 2.1 shows the seven internal trigger bits and the thresholds above which they are asserted.

TABLE 2.1. Internal bit triggers and their thresholds.

\begin{tabular}{|c|r|}
\hline Trigger bit & Threshold \\
\hline Det1 & $\mathrm{N}_{\text {tank }}>10$ \\
\hline Det2 & $\mathrm{N}_{\text {tank }}>24$ \\
\hline Det3 & $\mathrm{N}_{\text {tank }}>200$ \\
\hline Det4 & $\mathrm{N}_{\text {tank }}>100$ \\
\hline Det5 & $\mathrm{N}_{\text {tank }}>60$ \\
\hline Veto1 & $\mathrm{N}_{\text {veto }}<6$ \\
\hline Veto2 & $\mathrm{N}_{\text {veto }}<4$ \\
\hline
\end{tabular}

\subsection{Calibration System}

The data acquisition system records raw times and charges for each hit in an event, allowing for measurement of the intrinsic charge and time resolution of the PMTs without effects of smearing associated with the DAQ itself. These quantities, crucial for the event reconstruction and particle identification algorithms, are determined from calibration devices that include the laser calibration system, the cosmic ray muon tracker system and the scintillator cube system all built and operated by LSU. These systems were installed before I got to MiniBooNE, but I helped bring the cube system into operation. Subsequently, I worked on the maintenance of both the tracker and laser systems described below.

\subsubsection{Laser Calibration System}

The MiniBooNE laser calibration system consists of a pulsed diode laser and four dispersion flasks. Short pulses of laser light are transmitted via optical fibers to 


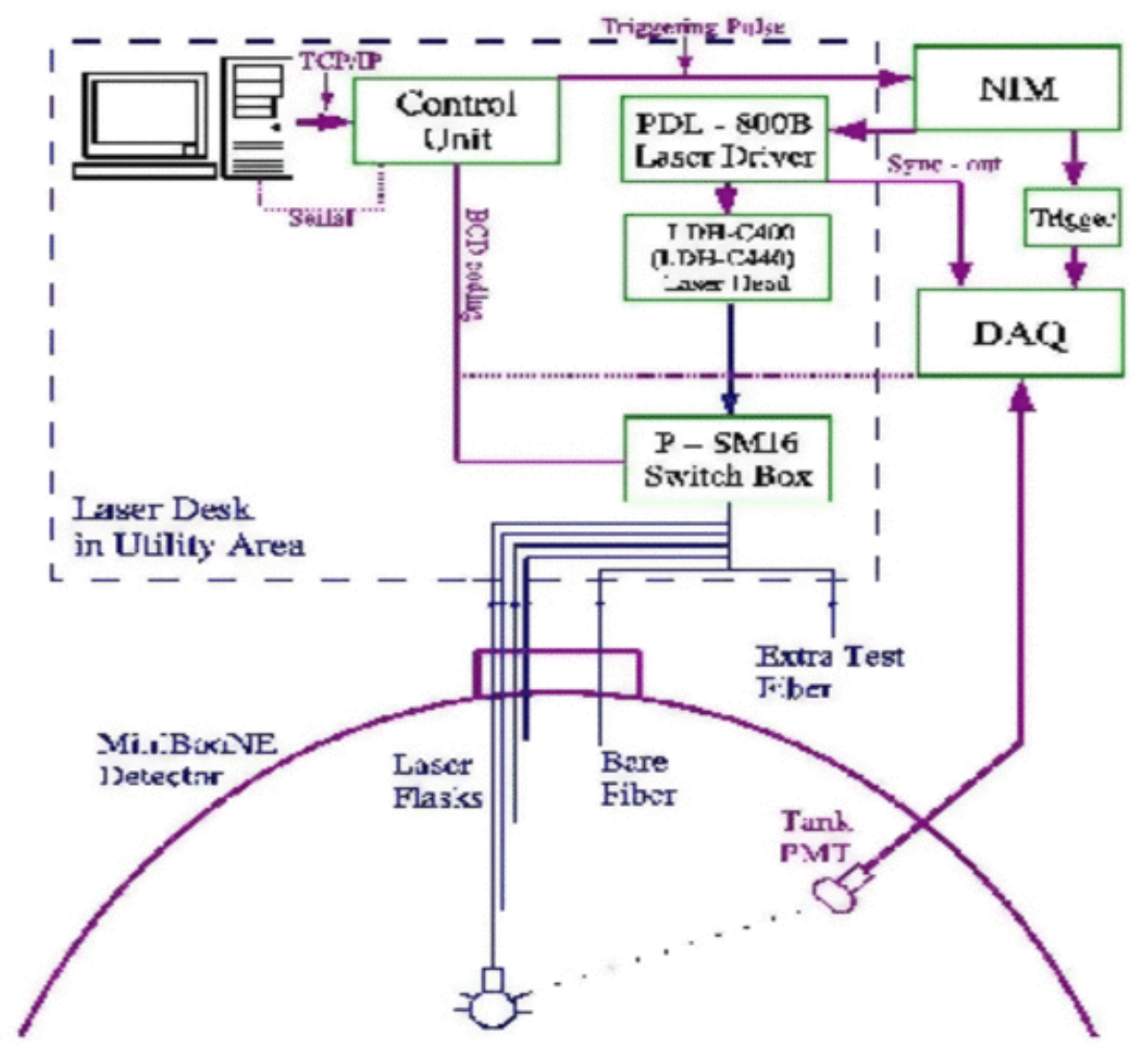

FIGURE 2.8. MiniBooNE laser calibration system

each of the dispersion flasks installed at various locations in the detector [34]. This system is used primarily to quantify and monitor individual PMT properties such as gain and timing. It also allows for in situ monitoring of the oil attenuation length over the lifetime of the experiment. Shown in figure 2.8 is a diagram of the laser/flask calibration system. The diode laser generates pulses with widths $<100$ ps. A switch box allows transmission of the laser light pulses to one of the four dispersion flasks via an optical fiber. The laser system is pulsed at 3.3 $\mathrm{Hz}$ continuously. Each dispersion flask is $10 \mathrm{~cm}$ in diameter, and filled with a dispersive medium called Ludox ${ }^{\circledR}$. Light from a flask illuminates all of the PMTs with roughly equal intensities. In addition to the four flasks, there is a bare optical 
TABLE 2.2. Flask positions in the MiniBooNE detector.

\begin{tabular}{|c||c|c|c|c|}
\hline Flask & $\mathrm{x}(\mathrm{cm})$ & $\mathrm{y}(\mathrm{cm})$ & $\mathrm{z}(\mathrm{cm})$ & radius $(\mathrm{cm})$ \\
\hline Flask 1 & -0.3 & -4.1 & 1.5 & 4.4 \\
\hline Flask 2 & 144.9 & 96.1 & -126.4 & 215.0 \\
\hline Flask 3 & 1.7 & -0.8 & 83.7 & 83.7 \\
\hline Flask 4 & -80.0 & 203.9 & -24.1 & 220.3 \\
\hline Bare fiber & 82.0 & 540.0 & 65.0 & 550.0 \\
\hline
\end{tabular}

fiber used to study light scattering in the detector. It emits light directly from the laser in a cone of $\sim 10^{\circ}$, illuminating PMTs in a small circle near the bottom of the detector. The locations of the four flasks and the bare fiber are shown in table 2.2 , where the origin is at the center of the detector, and positions are quoted in beam coordinates (z-axis along beam direction, y-axis toward detector top hat).

\subsubsection{PMTs Calibration}

The main signal region of the detector is instrumented with all of the R5912 PMTs and those R1408 PMTs whose test results showed the best performance. The remaining R1408 PMTs are mounted in the veto region, where performance requirements are less stringent. Each PMT in the signal region is affixed in its location with a wire frame whose feet are anchored to a phototube support structure (PSS). When a trigger condition is met, the following pieces of information are written to disk for each PMT channel:

- the clock tick number that occurred just before the discriminator fired (t-1 in figure 2.2)

- the charge quads (four digitized Vq values in ADC counts)

- the time quads (four digitized Vt values in ADC counts) 
The calibrated time and charge of a PMT hit are calculated as follow [35]:

$$
\begin{aligned}
T & =T_{\text {raw }}+T_{\text {slew }}\left(Q_{\text {raw }}\right)+T_{\text {offset }}+T_{\text {start }} \\
Q & =Q_{\text {raw }} / \text { Gain }
\end{aligned}
$$

where $\mathrm{T}_{\text {raw }}$ is the time (in ns) of the PMT hit relative to the preceding clock tick, and is obtained from the intersection of the slope of the ramping $V_{t}$ signal with the baseline. $\mathrm{T}_{\text {start }}$ is the number of the clock tick that precedes the firing of the discriminator, while $\mathrm{T}_{\text {offset }}$ is a calibration constant that accounts for the time delay caused by cable lengths and PMT dynode structures. The time slewing corrections, $\mathrm{T}_{\text {slew }}$, are needed to account for the fact that larger PMT signals fire the discrimnator earlier than small signals. These corrections are determined from different laser light intensity, and stored in look-up tables. For each PMT the gain, which converts ADC counts to number of photoelectrons, is obtained by fitting the single photoelectron $(\mathrm{PE})$ peak in low intensity laser runs.

\subsubsection{Cosmic Ray Muon Calibration System}

The cosmic ray muon calibration system [36] consists of a muon tracker located above the detector, and scintillator cubes located inside the detector. This system uses through-going cosmic ray muons as well as stopping muons to provide a sample

of particles with known direction and path length. A muon that stops in a cube and decays producing an electron, will have a distinct signature of two light pulses in time-delayed coincidence. Since the cubes are only a few centimeters on a side, the stopping position of these muons is known to an accuracy of a few centimeters. The starting position can be determined with similar accuracy using the muon tracker. 

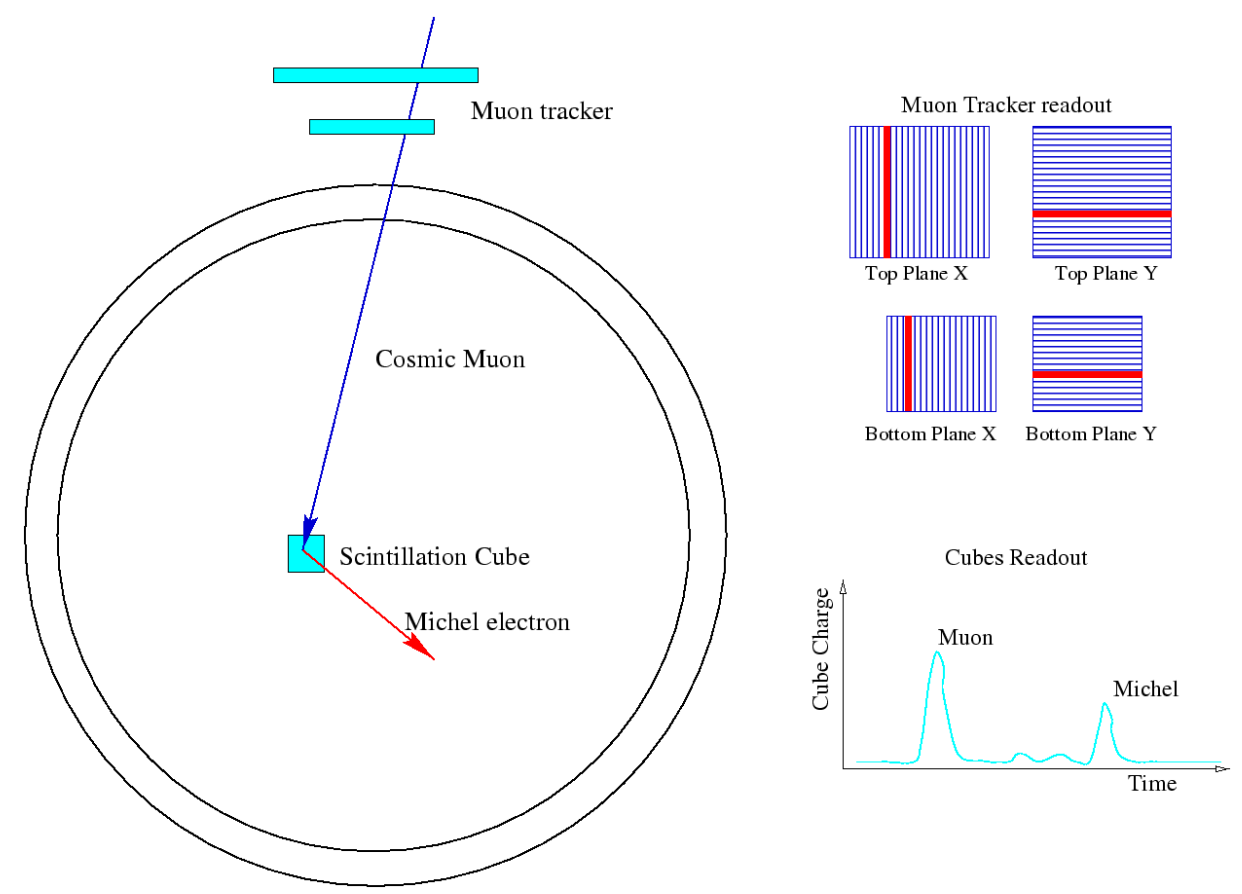

FIGURE 2.9. A schematic diagram of the muon tracker and a scintillation cube

\section{- Muon Tracker}

The muon tracker tags cosmic ray muons and records their positions and directions as they enter the tank. The muon tracker has two scintillator hodoscope modules each with a $\mathrm{X}$ and a $\mathrm{Y}$ layer [37]. Thus, it provides two sets of coordinates by which the position and direction of the muon can be determined. Each of the two top layers has 23 strips $10 \mathrm{~cm} \times 228 \mathrm{~cm} \times 1.59 \mathrm{~cm}$, while the two bottom layers, resting on the access portal, have 28 strips $6 \mathrm{~cm} \times 170 \mathrm{~cm} \times 1.59 \mathrm{~cm}$.

The muon tracker strips are fed into NIM logic circuits for triggering purposes. Each strip in a single plane is fed into an OR circuit, and the output of the four OR circuits are fed into an AND circuit. A muon tracker event requires all four planes to have a hit which is by definition a 4-fold coincidence in the AND circuit.

Hits on each strip are recorded in the form of uncalibrated data quads by the DAQ system. These hits are actually bit maps that tell which strips were hit. The raw data are reconstructed offline by a program called MuonTracer that is written 

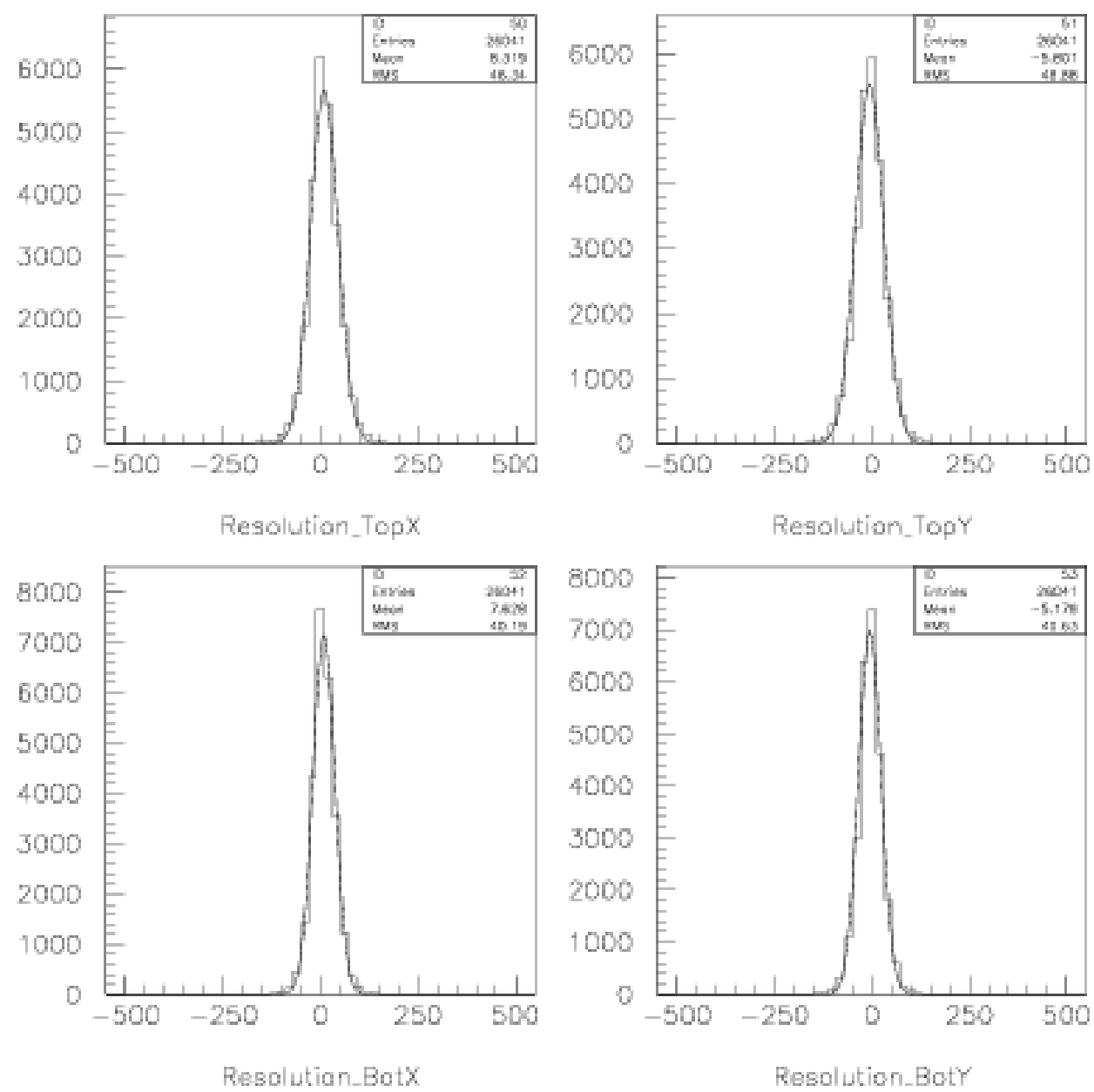

FIGURE 2.10. Position resolution of the four planes that constitute the muon tracker

to decode the bit maps and determines which strips were hit in a given event. It then reconstructs the direction and position of the muon when it enters the MiniBooNE detector.

The muon tracker is used to measure the angular and spatial resolution of contained cosmic ray muon events reconstructed with the Stancu fitting program that we will discuss in chapter 4. This study, performed by the LSU group [37] found the angular resolution to be better than $3^{\circ}$. Figure 2.10 shows the position resolution for each of the four planes of the tracker system. 
TABLE 2.3. Scintillator cube positions in beam coordinates ( $\mathrm{z}$ along beam, $\mathrm{y}$ toward tophat, $\tan (\phi)=\mathrm{z} / \mathrm{x})$.

\begin{tabular}{|c||c|c|c|c|}
\hline Cube & $\mathrm{x}(\mathrm{cm})$ & $\mathrm{y}(\mathrm{cm})$ & $\mathrm{z}(\mathrm{cm})$ & radius $(\mathrm{cm})$ \\
\hline \hline 1 & -18.6 & 371.2 & 59.2 & 376.4 \\
2 & 40.8 & 170.2 & 44.5 & 180.5 \\
3 & 40.8 & 273.9 & 44.5 & 280.5 \\
4 & 15.6 & 511.7 & -57.6 & 515.2 \\
5 & -60.8 & 540.7 & 15.1 & 544.3 \\
6 & -45.2 & 538.1 & -36.9 & 541.3 \\
7 & 57.9 & 471.5 & -13.5 & 475.2 \\
\hline
\end{tabular}

\section{- Cubes}

Seven optically isolated cubes made of plastic scintillator are situated at various depths in the main volume of the detector, providing additional information for those muons which traverse or stop in them. As given in table 2.3, the depth of the scintillator cubes varies from $15 \mathrm{~cm}$ to $400 \mathrm{~cm}$, so that muons with energy ranging from $20 \mathrm{MeV}$ to $800 \mathrm{MeV}$ can be observed stopping in a cube [38]. Approximately 100 to 500 muons per month stop in each cube, depending on its depth. Together with the Muon tracker, the scintillation cubes are used to performed a muon energy calibration. For a cosmic ray muon that goes through the muon tracker, enters the tank and stops in a cube, a three point fit to a straight line is performed using the two points from the muon tacker and the position of the cube [38]. The intersection of this line with the tank optical barrier gives the entry point of the muon in the detector. The muon range is the distance from the entry point to the cube position. The muon energy is determined using range values calculated with a Bethe-Bloch ionization energy loss $(\mathrm{dE} / \mathrm{dx})$ formula that includes delta rays for muons in mineral oil [39]. Figure 2.11 compares the energy of the cosmic ray muons that stop in cubes with the reconstructed energy using the standard 


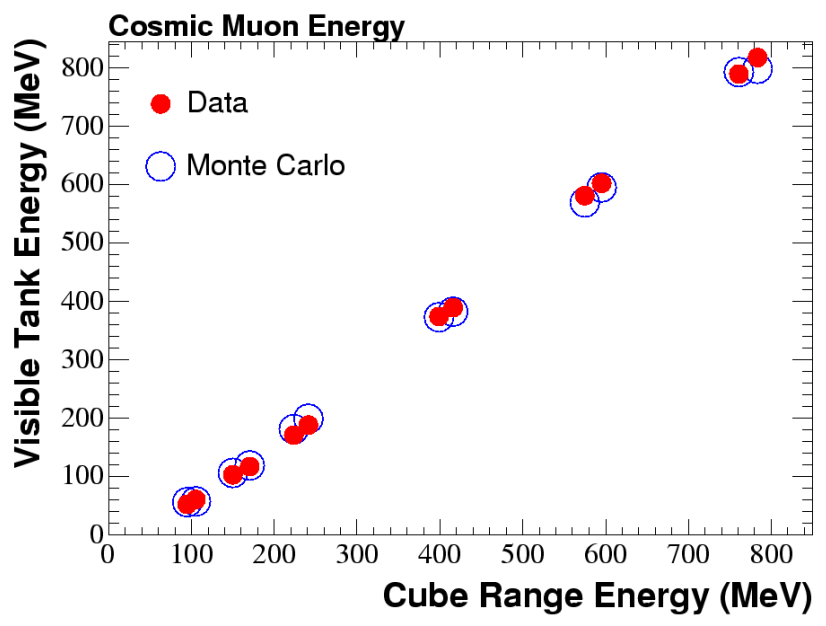

FIGURE 2.11. Muon energy determined by the reconstruction vs. cube range energy calculated from the muon path obtained from the muon tracker and the scintillator cubes inside the tank. Data is shown by points, Monte Carlo is the solid histogram.

event fitter algorithm. The uncertainty in the muon energies in this sample is almost entirely due to range straggling, and the absolute energy determination for these events is approximately $2 \%$ for $\mathrm{E}_{\mu}>200 \mathrm{MeV}$ [38]. The distribution in the cosine of the angle between the muon direction and a line from the track midpoint to the hit PMTs for stopping muons is shown in figure 2.4.3. The top plot on the left shows the cosine distribution for muons that stop in the $30 \mathrm{~cm}$ cube. Both data and Monte Carlo distributions peak around 0.82 which is the expected opening angle of the $\check{C}$ erenkov cone for muons with kinetic energy $\sim 100$ $\mathrm{MeV}$. For the cube at $60 \mathrm{~cm}$ (top right), the $\check{C}$ erenkov cone is shifted slightly to $\sim 0.75$, as expected for muons with kinetic energy around $150 \mathrm{MeV}$. Observation of the remaining plots shows that the trend continues as the cubes get deeper. In the deepest cubes, the peak becomes very broad because the path length of the muon is longer, and the assumption of photon emission from the track midpoint is no longer a good approximation [38]. The broad background in each distribution is due to scintillation light and fluorescence of $\check{C}$ erenkov light coupled with the geometry of the cubes. 

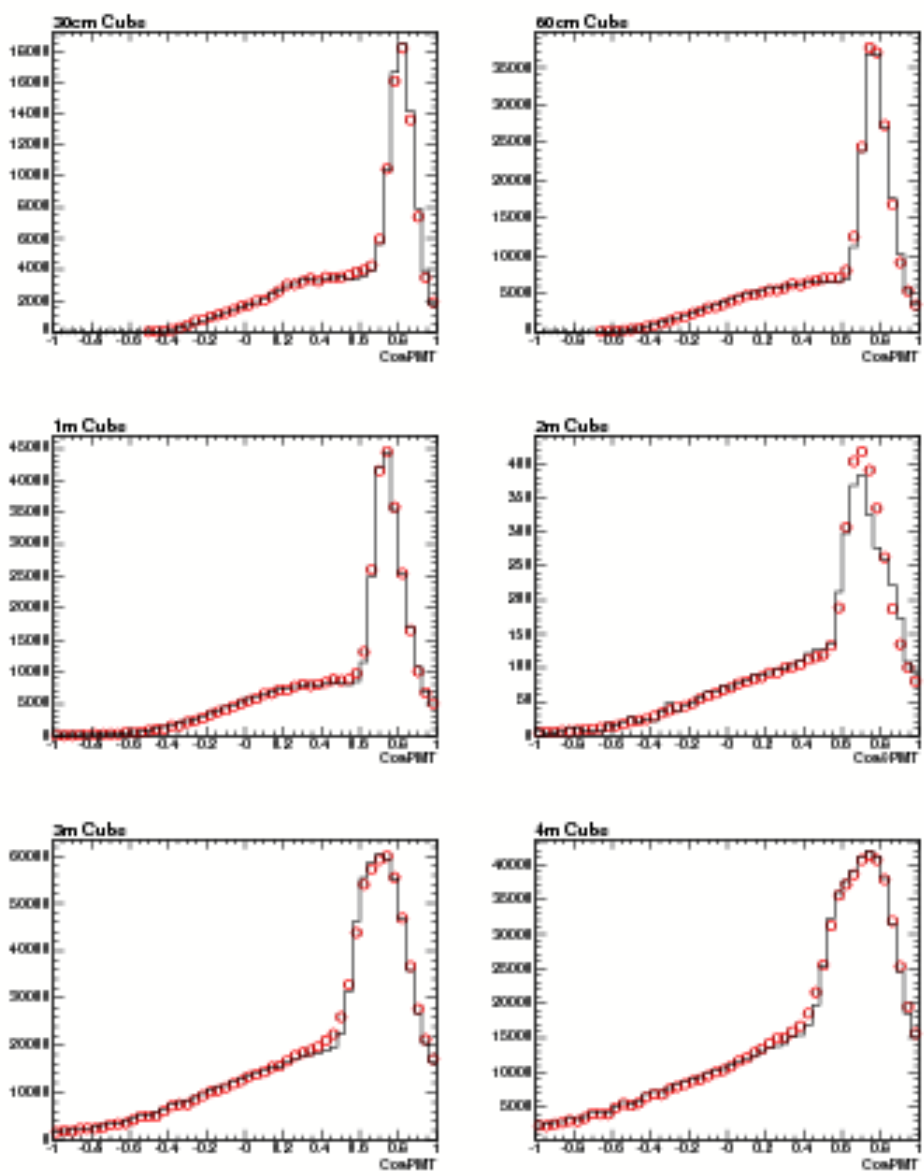

FIGURE 2.12. Corrected angle distributions of tank PMT hits for stopping muon events in the six deepest cubes. The event vertex and time are measured using the cubes and muon tracker. Data is shown by points, Monte Carlo is the solid histogram. 


\section{Chapter 3}

\section{Experiment Simulation}

\subsection{Overview}

MiniBooNE uses a Geant4-based Monte Carlo program [40], to simulate particle production in $\mathrm{p}$-Be interactions in the target and the propagation of the secondary particles through the target and decay region as described in chapter 2 . The resulting predicted neutrino fluxes are passed to the Nuance event generator [41], which integrates the neutrino cross sections over the flux and generates events. The interactions generated by Nuance are input to a Geant3-based [42] Monte Carlo program that simulates the detector. Finally, a simulation of the DAQ takes the events from the detector Monte Carlo as input and produces events that are identical to MiniBooNE data.

\subsection{Interactions in the Beryllium Target}

\subsubsection{Inelastic Interactions}

Inelastic interactions of the incident protons in the Beryllium target result in the production of new particles, in particular mesons. The simulation of interactions of $8.9 \mathrm{GeV} / \mathrm{c}$ protons on beryllium uses a total inelastic cross section of $\sigma_{\text {inel }}=189.3$ $\mathrm{mb}$, that was obtained from an interpolation of the cross-sections measured in the BNL E910 experiment at 6.4 and $12.3 \mathrm{GeV} / \mathrm{c}$ proton beam momenta [43]. The mesons produced in the inelastic proton-beryllium interactions of most relevance for neutrino fluxes are: $\pi^{+}, \pi^{-}, K^{+}, K^{0}$. For each type of meson, the number of particles produced in a given inelastic interactaion is chosen from a Poisson 
distribution with mean:

$$
N=\frac{\iint \frac{d^{2} \sigma}{d p d \Omega} d p d \Omega}{\sigma_{\text {ine }}}
$$

where $\frac{d^{2} \sigma}{d p d \Omega}$ is the meson production double differential cross section for that meson, and $\sigma_{i n e}$ is total proton-Beryllium inelastic cross section. At MiniBooNE $\pi^{+}, \pi^{-}$ and $\mathrm{K}^{0}$ production are well described by a Sanford-Wang parametrization [44] :

$$
\frac{d^{2} \sigma}{d p d \Omega}=c_{1} p^{c_{2}}\left(1-\frac{p}{p_{B}-c_{9}}\right) \exp \left[-c_{3} \frac{p^{c_{4}}}{p_{B}^{c_{5}}}-c_{6} \theta\left(p-c_{7} p_{B} \cos ^{c_{8}} \theta\right)\right]
$$

where $\mathrm{p}_{B}$ is proton momentum, $\mathrm{p}$ is the outgoing meson momentum given in $\mathrm{GeV} / \mathrm{c}$ and $\theta$ is the angle in radians of the outgoing meson with respect to the proton direction.

The HARP experiment [45] measured the double differential cross section of the interaction of protons at $\mathrm{p}_{B}=8.9 \mathrm{GeV} / \mathrm{c}$ on a Beryllium target similar to the MiniBooNE target. The data from this experiment are used in $\chi^{2}$ minimization fits to determine the coefficients $c_{1}$ through $c_{8}$ for each particle type. Also, $c_{9}$ was set to one for both $\pi^{+}$and $\pi^{-}$.

The results of the fits are listed in table 3.1 while figure 3.1 shows the best fit Sanford-Wang function along with HARP data for $\pi^{+}$production.

TABLE 3.1. Sanford-wang parameters for $\pi^{+}, \pi^{-}, K^{0}$ production

\begin{tabular}{|c|ccccccccc}
\hline & $\mathrm{c}_{1}$ & $\mathrm{c}_{2}$ & $\mathrm{c}_{3}$ & $\mathrm{c}_{4}$ & $\mathrm{c}_{5}$ & $\mathrm{c}_{6}$ & $\mathrm{c}_{7}$ & $\mathrm{c}_{8}$ & $\mathrm{c}_{9}$ \\
\hline$\pi^{+}$ & 220.7 & 1.080 & 1 & 1.978 & 1.32 & 5.572 & 0.08678 & 9.861 & 1 \\
$\pi^{-}$ & 237.2 & 0.8986 & 4.521 & 1.154 & 1.105 & 4.224 & 0.06613 & 9.961 & 1 \\
$\mathrm{~K}^{0}$ & 15.13 & 1.975 & 4.084 & 0.9277 & 0.7306 & 4.362 & 0.04789 & 13.3 & 1.278 \\
\hline
\end{tabular}

The simulation of the $\mathrm{K}^{+}$production is described with a Feynman Scaling parametrization where the invariant cross section is only dependent of the trans- 


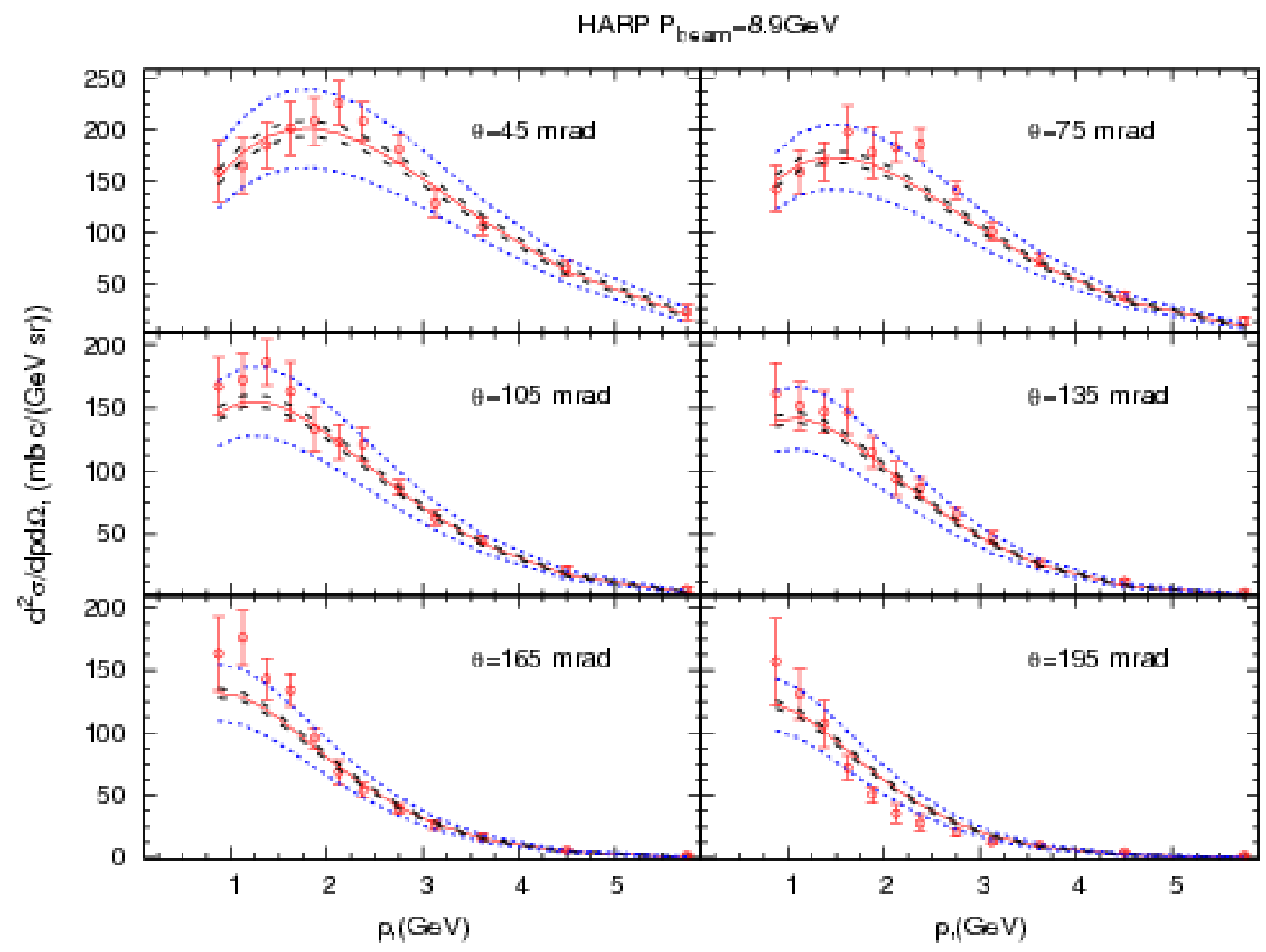

FIGURE 3.1. A plot showing HARP results for $\mathrm{pBe} \rightarrow \pi^{+} \mathrm{X}$ interaction. The Sanford-Wang function is the red curve and the HARP data are the red points. The blue curves represent the uncertainties.

verse momentum $\mathrm{p}_{t}$ and the Feynman $x_{F}$ defined as:

$$
x_{F}=\frac{P_{\|}^{C M}}{P_{\|}^{C M, M a x}}
$$

where $P_{\|}^{C M}$ is the longitudinal momentum of the meson in the center-of-mass frame and $P_{\|}^{C M, M a x}$ is its maximum possible value. The Feynman Scaling parametrization is given by:

$$
\frac{d^{2} \sigma}{d p d \Omega}=\frac{p^{2}}{E} c_{1} \exp \left[-c_{3}\left|x_{F}\right|^{c_{4}}-c_{7}\left|p_{t} x_{f}\right|^{c_{6}}-c_{2} p_{t}-c_{5} p_{t}^{2}\right]
$$


where $\mathrm{p}$ and $\mathrm{E}$ are the momentum and the energy of the $\mathrm{K}^{+}$. Here, $\mathrm{c}_{1}$ through $\mathrm{c}_{7}$ are determined from fits to external data listed in table 3.2. Table 3.3 lists the best fit Feynman Scaling parameters and figure 3.2 compares the resulting function with the data.

TABLE 3.2. $\mathrm{K}^{+}$production data used for the Feynman scaling fits. Data with $1.2 \mathrm{GeV} / \mathrm{c}$ $<\mathrm{p}<5.5 \mathrm{GeV} / \mathrm{c}$ are used. The data from Vorontsov were not used because of a large normalization offset compared to the data from the other sources.

\begin{tabular}{|c|c|c|c|c|c|}
\hline Experiment & $\mathrm{p}_{\text {proton }}(\mathrm{GeV} / \mathrm{c})$ & $\mathrm{p}_{K}(\mathrm{GeV} / \mathrm{c})$ & $\Theta_{K}$ & $\mathrm{~N}_{\text {DATA }}$ & $\sigma_{\text {NORM }}$ \\
\hline Abbott [46, 39, 47] & 14.6 & $2-8$ & $20-30^{\circ}$ & 43 & $10 \%$ \\
Aleshin [46, 39] & 9.5 & $3-6.5$ & $3.5^{\circ}$ & 5 & $10 \%$ \\
Eichten [46, 39, 48] & 24.0 & $4-18$ & $0-6^{\circ}$ & 56 & $20 \%$ \\
Piroue [46, 39, 49] & 2.74 & $0.5-1$ & $13,30^{\circ}$ & 13 & $20 \%$ \\
Vorontsov [46, 39] & 10.1 & $1-4.5$ & $3.5^{\circ}$ & 13 & $25 \%$ \\
\hline
\end{tabular}

TABLE 3.3. Feynman scaling parameters for $\mathrm{K}^{+}$production.

\begin{tabular}{|c|c|c|c|c|c|c|c|}
\hline & $\mathrm{c}_{1}$ & $\mathrm{c}_{2}$ & $\mathrm{c}_{3}$ & $\mathrm{c}_{4}$ & $\mathrm{c}_{5}$ & $\mathrm{c}_{6}$ & $\mathrm{c}_{7}$ \\
\hline $\mathrm{K}^{+}$ & 11.70 & 0.88 & 4.77 & 1.51 & 2.21 & 2.17 & 1.51 \\
\hline
\end{tabular}

\subsubsection{Elastic And Quasi-Elastic Interactions}

Particles in the target can also interact elastically. Elastic interactions are processes where the incident proton scatters coherently off of the target nucleus or nucleon. These processes affect the direction of the proton, while the energy of both the proton and the struck particle remain the same, with no particle absorption, particle production or charge exchange.

For quasi-elastic processes, however, the incident proton scatters incoherently off a nucleon. The total number of particles is conserved, but momentum and quantum numbers can be exchanged. From the Monte Carlo simulation, quasielastic interactions are responsible for $\sim 5 \%$ of the neutrino flux incident on the 

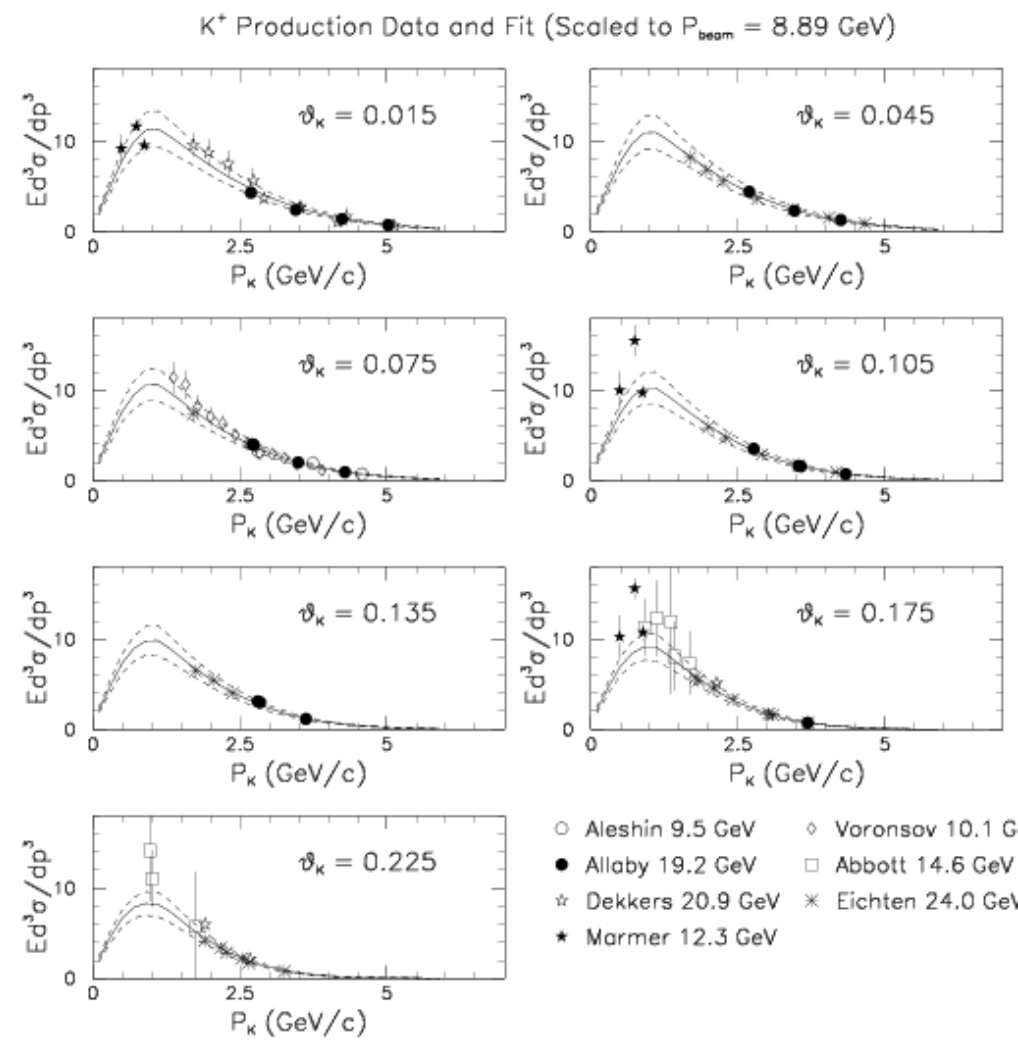

- Aleshin $9.5 \mathrm{GeV}$ Voronsov $10.1 \mathrm{GeV}$

- Allaby $19.2 \mathrm{GeV} \square$ Abbott $14.6 \mathrm{GeV}$

น́ Dekkers $20.9 \mathrm{GeV}$ * Eichten $24.0 \mathrm{GeV}$

* Marmer $12.3 \mathrm{GeV}$

FIGURE 3.2. Feynman scaling parametrization (solid curve), error bands (dashed curve), and data (points) for the $\mathrm{K}^{+}$production.

MiniBooNE detector, and elastic interactions contribute $<1 \%$ due to their effect on the energy and angular distributions of subsequent inelastic interactions.

\subsection{Electromagnetic Processes}

The mesons generated from the pBe interactions are tracked through a simulation of the beam line geometry, that includes a description of the trajectory of the particles in the horn, ionization energy loss, multiple Coulomb scattering, and meson decay.

The neutrino rate per proton-on-target is increased by a factor of six during horn-on running compared to horn-off running [50, 51]. Since this increase is due to the focusing effect on positively charged particles by the horn magnetic field, it 
is important to properly simulate the trajectory of charged particles in this field, in order to obtain reliable flux predictions.

Charged particles traversing a material will experience Coulomb forces resulting in many small electromagnetic scatters. The pion and proton angular distributions from multiple Coulomb scattering are fully simulated in the beryllium and aluminum target material [51]. The beam Monte Carlo also simulates the continuous energy loss due to ionization and atomic excitation.

\subsection{Neutrino Flux}

The predicted neutrino fluxes are shown as a function of neutrino energy in figure 3.3. According to the Monte Carlo, the neutrino beam composition is $\sim 93.5 \% \nu_{\mu}$, $5.9 \% \overline{\nu_{\mu}}, 0.5 \% \nu_{e}$, and $0.1 \% \overline{\nu_{e}}$. The $\nu_{\mu}$ flux is $\sim 97 \%$ from $\pi^{+}$decay, and $\sim 3 \%$ from $K^{+}$decay. The $\nu_{e}$ flux is about $38 \%$ from $K^{+}$decay, $\sim 7 \%$ from $K_{L}^{0}$ decay, and $52 \%$ from $\mu^{+}$decay [34]. The detailed meson parentage history of the flux by neutrino flavor is summarized in table 3.4

\subsection{Neutrino Cross Section Model}

Nuance is a FORTRAN-based Monte Carlo program that takes as input the predicted fluxes $\left(\nu_{\mu}, \bar{\nu}_{\mu}, \nu_{e}, \bar{\nu}_{\mu}\right)$ as described in section 3.4 to simulate the neutrino interaction cross sections and final state kinematics. NUANCE is an open source code that was developed to simulate atmospheric neutrino interactions in the IMB detector. The code has later been generalized and is used by several neutrino experiments other than MiniBooNE, including Super-Kamiokande, K2K, SNO, and KamLAND. At MiniBooNE, most of the interactions are due to charged current quasi-elastic scattering (39\%), charged current resonant $\pi^{+}$production $(25 \%)$, neutral current elastic scattering $(16 \%)$, and neutral current $\pi^{0}$ production $(8 \%)$. The 

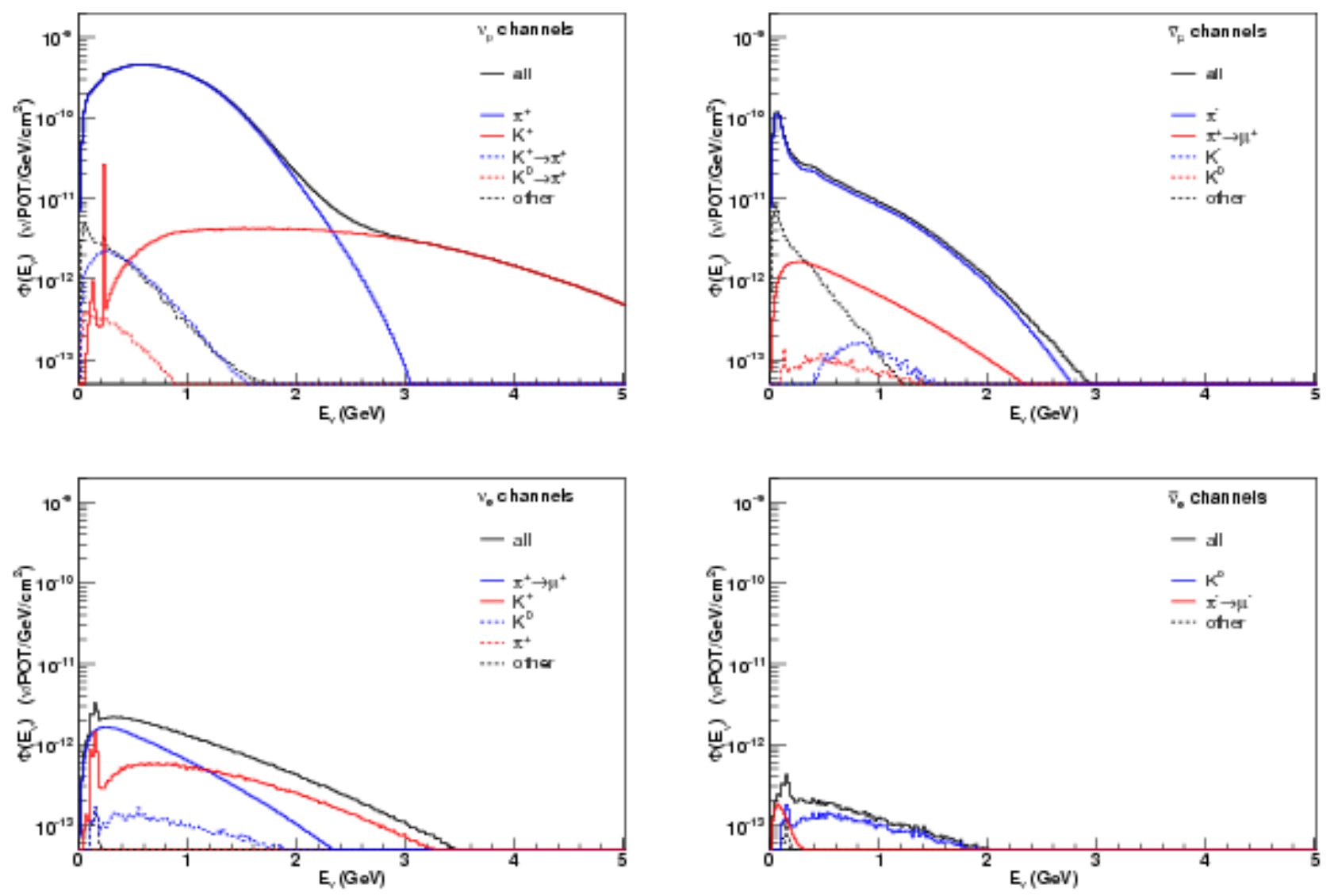

FIGURE 3.3. Predicted neutrino flux in the MiniBooNE detector for each neutrino type.

rate of the $\nu_{\mu}$ e elastic scatters, described in the next section, is important for this thesis but represent a small fraction of the interactions.

\subsubsection{Simulation of Neutrino Electron Scattering}

The $\nu_{\mu}+e \rightarrow \nu_{\mu}+e$ process is characterized by an exchange of a neutral boson $\mathrm{Z}^{0}$. The Lagrangian is given by:

$$
\mathcal{L}=-\frac{G_{F}}{\sqrt{2}}\left[\bar{\nu}_{\mu} \gamma^{\mu}\left(1-\gamma_{5}\right) \nu_{\mu}\right]\left[\bar{e} \gamma_{\mu}\left(g_{V}-g_{A} \gamma_{5}\right) e\right]
$$

which rewritten in term of the weak coupling constants $\mathrm{g}_{L}$ and $\mathrm{g}_{R}$ is:

$$
\mathcal{L}=-\frac{G_{F}}{\sqrt{2}}\left[\bar{\nu}_{\mu} \gamma^{\mu}\left(1-\gamma_{5}\right) \nu_{\mu}\right]\left[g_{L} \bar{e} \gamma_{\mu}\left(1-\gamma_{5}\right)+g_{R} \gamma_{\mu}\left(1+\gamma_{5}\right)\right]
$$


TABLE 3.4. MiniBooNE Geant4 beam Monte Carlo neutrino flux production modes.

\begin{tabular}{|c|c|c|}
\hline Neutrino Flavor & Process & Predicted Fraction \\
\hline \multirow[t]{7}{*}{$\nu_{\mu}(93.5 \%)$} & $\pi^{+}$ & $96.72 \%$ \\
\hline & $K^{+}$ & $2.65 \%$ \\
\hline & $K^{+} \rightarrow \pi^{+}$ & $0.26 \%$ \\
\hline & $K^{0} \rightarrow \pi^{+}$ & $0.04 \%$ \\
\hline & $K^{0}$ & $0.03 \%$ \\
\hline & $\pi^{-} \rightarrow \mu^{-}$ & $0.01 \%$ \\
\hline & others & $0.30 \%$ \\
\hline \multirow[t]{8}{*}{$\bar{\nu}_{\mu}(5.9 \%)$} & $\pi^{-}$ & $89.74 \%$ \\
\hline & $\pi^{+} \rightarrow \mu^{+}$ & $4.54 \%$ \\
\hline & $K^{+}$ & $0.51 \%$ \\
\hline & $K^{0}$ & $0.44 \%$ \\
\hline & $K^{0} \rightarrow \pi^{-}$ & $0.24 \%$ \\
\hline & $K^{+} \rightarrow \mu^{+}$ & $0.06 \%$ \\
\hline & $K^{-} \rightarrow \mu^{-}$ & $0.03 \%$ \\
\hline & others & $4.43 \%$ \\
\hline \multirow[t]{6}{*}{$\nu_{e}(0.5 \%)$} & $\pi^{+} \rightarrow \mu^{+}$ & $51.64 \%$ \\
\hline & $K^{+}$ & $37.80 \%$ \\
\hline & $K_{L}^{0}$ & $7.39 \%$ \\
\hline & $\pi^{+}$ & $2.16 \%$ \\
\hline & $K^{+} \rightarrow \mu^{+}$ & $0.69 \%$ \\
\hline & others & $0.84 \%$ \\
\hline \multirow[t]{6}{*}{$\bar{\nu}_{e}(0.1 \%)$} & $K_{L}^{0}$ & $70.65 \%$ \\
\hline & $\pi^{-} \rightarrow \mu^{-}$ & $19.33 \%$ \\
\hline & $K^{-}$ & $4.07 \%$ \\
\hline & $\pi^{-}$ & $1.26 \%$ \\
\hline & $K^{-} \rightarrow \mu^{-}$ & $0.07 \%$ \\
\hline & others & $4.62 \%$ \\
\hline
\end{tabular}

where $g_{L}=-1 / 2+\sin ^{2} \theta_{w}$ and $g_{R}=\sin ^{2} \theta_{w}$. The Weinberg angle $\theta_{w}$ has been measured by several experiments $[6,7]$ and their reported values are in good agreement with the Standard Model prediction.

After detailed calculations [52], the partial differential cross section of the interaction in the Standard Model can be written as:

$$
\frac{d \sigma}{d y}=\frac{G_{F}^{2}}{4 \pi} \frac{m_{Z}^{2}}{q^{2}-m_{z}^{2}} s\left[g_{L}{ }^{2}+g_{R}{ }^{2}(1-y)^{2}+g_{L} g_{R} \frac{m_{e} y}{E_{\nu}}\right]
$$


where $\mathrm{m}_{Z}=91.1876 \pm 0.0021 \mathrm{GeV}$ is the mass of the $\mathrm{Z}^{0}$ boson, $\mathrm{s}=\left(\mathrm{p}_{\nu}+\mathrm{p}_{e}\right)^{2}$ is the square of the center of mass energy, and $\mathrm{q}^{2}=\left(\mathrm{p}_{\nu}-\mathrm{p}_{e}\right)^{2}$ is the momentum transfer with $\mathrm{p}_{\nu}$ and $\mathrm{p}_{e}$ the respective four-momentum of the neutrino and the electron. The quantity y is called the inelasticity or Bjorken variable and corresponds to:

$$
y=\frac{p_{e} \cdot\left(p_{\nu}-p_{e}\right)}{p_{e} \cdot p_{\nu}}=\frac{E_{\nu}-E_{e}}{E_{\nu}} .
$$

For MiniBooNE data $\mathrm{q}^{2}<<\mathrm{m}_{Z}^{2}$, and $\mathrm{m}_{e}<<\mathrm{E}_{\nu}$, thus an integration of the partial cross section over y gives:

$$
\sigma=\frac{G_{F}^{2} s}{4 \pi}\left[g_{L}^{2}+\frac{1}{3} g_{R}^{2}\right]
$$

Given that $\mathrm{s}=2 \mathrm{~m}_{e} \mathrm{E}_{\nu}$, the weak interaction cross section of the $\nu_{\mu}+e \rightarrow \nu_{\mu}+e$ interaction is [7]:

$$
\frac{\sigma}{\left\langle E_{\nu}\right\rangle}=1.80 \times 10^{-42} \mathrm{~cm}^{2} / \mathrm{GeV}
$$

Elastic scattering events play an important role because they provide a clean sample of events with a well-understood cross section whose theoretical uncertainty is less than 1\% [7]. In Chapter 5 we will detail a search for the $\nu_{\mu}$ magnetic moment using this process. Neutrino electron scattering is included in NUANCE at the tree level with the Standard Model assumption of a non-existent neutrino magnetic moment.

\subsubsection{Simulation of Charged Current Quasi-Elastic Interaction}

Charged Current Quasi-Elastic (CCQE) scattering $\nu_{l}+\mathrm{n} \rightarrow l^{-}+\mathrm{p}$ is the dominant process at MiniBooNE. The CCQE cross section is given by:

$$
\frac{d \sigma}{d Q^{2}}=\frac{M^{2} G_{F}\left|V_{u d}\right|^{2}}{8 \pi E_{\nu}^{2}}\left[A\left(Q^{2}\right) \pm B\left(Q^{2}\right) \frac{s-u}{M^{2}}+C\left(Q^{2}\right) \frac{(s-u)^{2}}{M^{4}}\right]
$$


where $\mathrm{M}$ is th mass of the target nucleon, $\mathrm{Q}^{2}$ is the negative four-momentum transfer: $\mathrm{Q}^{2}=-\mathrm{q}^{2}=\left(\mathrm{p}_{\nu}-\mathrm{p}_{\mu}\right)^{2}, \mathrm{G}_{F}$ is the Fermi constant, $\mathrm{V}_{u d}$ represents the CKM matrix element and $\mathrm{E}_{\nu}$ is the incident neutrino energy. The quantity $(s-u)=$ $4 M E_{\nu}-Q^{2}$ uses the usual Mandelstam variables:

$$
\begin{aligned}
s & =p_{\nu}+p_{n} \\
u & =p_{\nu}-p_{p}
\end{aligned}
$$

where $\mathrm{p}_{\nu}, \mathrm{p}_{n}, \mathrm{p}_{p}$ are repectively the four-momentum of the neutrino, neutron and proton.

$\mathrm{A}\left(\mathrm{Q}^{2}\right), \mathrm{B}\left(\mathrm{Q}^{2}\right)$ and $\mathrm{C}\left(\mathrm{Q}^{2}\right)$ contain information related to the nucleon structure and are functions of the Dirac isovector $\mathrm{F}_{V}$, the Pauli isovector $\mathrm{F}_{M}$, the axial vector $\mathrm{F}_{A}$ and the pseudoscalar $\mathrm{F}_{P}$ form factors [53]. The form factors $\mathrm{F}_{V}$ and $\mathrm{F}_{M}$ are related to the electromagnetic form factors $\left(G_{E}, G_{M}\right)$ and are parametrized in terms of a mass $\mathrm{M}_{V}$ obtained from elastic scattering experiments. The axial form factor $\mathrm{F}_{A}$ is parametrized in term of an axial mass parameter $\mathrm{M}_{A}=1.25 \pm 0.12$ $\mathrm{GeV}^{2}$ for $\mathrm{Q}^{2}>0.25 \mathrm{GeV}^{2}$, obtained from the MiniBooNE CCQE data [21].

\subsubsection{Simulation of Charged Current Single Pion production}

In chapter 1 , it was noted that the differential cross section for the production of a nucleon resonance in a neutrino interaction is:

$$
\frac{d \sigma}{d q^{2} d W}=\frac{1}{32 \pi m_{N} E_{\nu}^{2}} \frac{1}{2} \sum_{\text {spins }}\left|M\left(\nu_{\mu}+N \rightarrow \nu_{\mu}+N^{\star}\right)\right|^{2} \frac{1}{2 \pi} \frac{\Gamma}{\left(W-m_{N}\right)^{2}+\Gamma^{2} / 4}(3
$$

NUANCE models resonances with invariant mass $\mathrm{W}=\left(p_{N}+p_{\pi}\right)^{2}<2 \mathrm{GeV}$ using the Rein Sehgal resonance cross section model [54]. In the model, the cross section for each state is calculated as a superposition of all the possible contributing resonances. Rein and Sehgal use the relativistic harmonic oscillator quark model of 
Feynman, Kislinger, and Ravndal [55] to calculate the transition matrix elements $M\left(\nu_{\mu}+N \rightarrow \nu_{\mu}+N^{\star}\right)$ from a ground state nucleon to a baryon resonance. The model for the decay of each resonance to a final state uses experimental input for resonance mass, resonance width, and branching ratios.

\subsubsection{Nuclear Effects}

In most charged current interactions in MiniBooNE, the neutrino interacts with a target nucleon bound inside a carbon nucleus, in which case three additional factors need to be taken into consideration:Fermi motion of the target nucleons, Pauli suppression of the phase space available to final state protons, and final state interactions (FSI). These factors, known as the nuclear effects are discussed below.

\section{- Pauli Blocking And Fermi Motion}

NUANCE uses the Smith-Moniz relativistic Fermi gas model to address the Fermi motion of the neutron. In the Smith and Moniz formalism, the target nucleons are assumed to have a uniform momentum density up to the Fermi momentum $\mathrm{p}_{F}=220 \mathrm{MeV} / \mathrm{c}$ for ${ }^{12} \mathrm{C}$, and a nucleon binding energy $\mathrm{E}_{b}=34 \mathrm{MeV}$. The Pauli blocking requires $\left|\overrightarrow{p_{n}}\right|<p_{F}$ and $\left|\overrightarrow{p_{p}}\right|>p_{F}$, where $\left|\overrightarrow{p_{p}}\right|$ is the momentum of the final state proton and $\left|\overrightarrow{p_{n}}\right|$ the momentum of the initial neutron, resulting in a suppression of the CCQE and $\mathrm{CC} \pi^{+}$cross sections especially at low neutrino energies.

Taking into account the Fermi motion and the binding energy, CCQE interactions $\left(\nu_{\mu}+n \rightarrow \mu^{-}+p\right)$ occur only when the energy transfer $\Delta E=E_{\nu}-E_{\mu}$ is greater than the binding energy $\mathrm{E}_{b}$, with $E_{\nu}$ and $E_{\mu}$ being respectively the neutrino and the muon energy. Moreover, the momentum transfer $Q^{2}=-\left(p_{\nu}-p_{\mu}\right)^{2}$, which is equal to $2 m_{N} \Delta E$ if the Fermi motion and binding energy are neglected, takes a range of values around this central value. 


\section{- Final State Interactions}

Final state hadrons produced in neutrino interactions can interact with nucleons within the nucleus in which they are produced. These interactions which include charge exchange, pion absorption, elastic and inelastic scattering, will affect the composition and the kinematics of the final states particles.

\subsection{Detector Simulation}

The final state particles produced by the NUANCE event generator [41] are passed to a GEANT3 [42] based Monte Carlo simulation that models the transport of the particles in each event across the oil medium with the production and propagation of individual photons which are tracked from their production point until they leave the detector, hit a PMT or are absorbed in the detector material.

\subsubsection{Light Production in the Detector}

Charged particles propagating through the mineral oil produce optical photons which are individually tracked in the Monte Carlo. The primary means of particle detection in MiniBooNE is via Čerenkov radiation by charged particles travelling faster than the speed of light in the oil. The other source of light production is scintillation. A full understanding of the detector response requires also an understanding of the various processes that the optical photons undergo prior to detection. These include scattering, absorption and fluorescence.

\section{- Čerenkov Light Production}

$\breve{C}$ erenkov light is prompt in time. It is produced in a medium with index of refraction $\mathrm{n}$, when a charged particle travels faster than the speed of light in that medium. A particle travelling with a speed $\beta>1 / \mathrm{n}$ produces a shock wave of photons which are radiated in a cone with a characteristic opening angle with respect 
to the particle track given by $\cos \left(\theta_{C}\right)=1 /(n \beta)$. The number of $\check{C}$ erenkov photons emitted per unit path length in the detector is given by:

$$
\frac{d^{2} N}{d x d \lambda}=\frac{2 \pi \alpha z^{2}}{\lambda^{2}}\left(1-\frac{1}{\beta^{2} n^{2}}\right)=\frac{2 \pi \alpha z^{2}}{\lambda^{2}} \sin ^{2} \theta
$$

where $\alpha$ is the fine structure constant, $\mathrm{z}$ the particle charge, and $\lambda$ the wavelength of the emitted light.

The $\check{C}$ erenkov light also depends on the wavelength dependence of the index of refraction which is a measurement of the dielectric properties of the medium of propagation. This dependence is well described for MiniBooNE oil by:

$$
n(\lambda)=\left[n+B\left(\frac{1}{\lambda^{2}}-\frac{1}{\lambda_{D}^{2}}\right)\right]
$$

where $\lambda$ is the photon wavelength, $\lambda_{D}=589.3 \mathrm{~nm}$ and the parameter $\mathrm{B}=4240 \pm$ $157 \mathrm{~nm}^{2}$. Using the measured value of $\mathrm{n}=1.4684 \pm 0.0002$ in the oil, the $\breve{C}$ erenkov angle for $\beta=1$ is calculated to be $47^{\circ}$.

\section{- Scintillation Light Production}

Other than $\check{C}$ erenkov light, charged particles traveling through mineral oil radiate energy which excites electron states of oil molecules. The isotropic, delayed light that is emitted during de-excitation of the molecules is called scintillation light. The scintillation properties of the MiniBooNE oil have been measured with 180 $\mathrm{MeV}$ kinetic energy protons at the Indiana University cyclotron.

\subsubsection{Light Transmission}

Optical photons propagating from their emission point can be attenuated before reaching their detection point at the PMT surface. Attenuation is a physical process that includes both photon absorption, scattering, and fluorescence. The number of photons remaining after traveling a distance $\mathrm{x}$ is given by: $\mathrm{N}(\mathrm{x})=\mathrm{N}_{0} \exp \left[-\mathrm{x} / \lambda_{A}\right]$, 


\section{Extinction Rate for MiniBooNE Marcol 7 Mineral Oil}

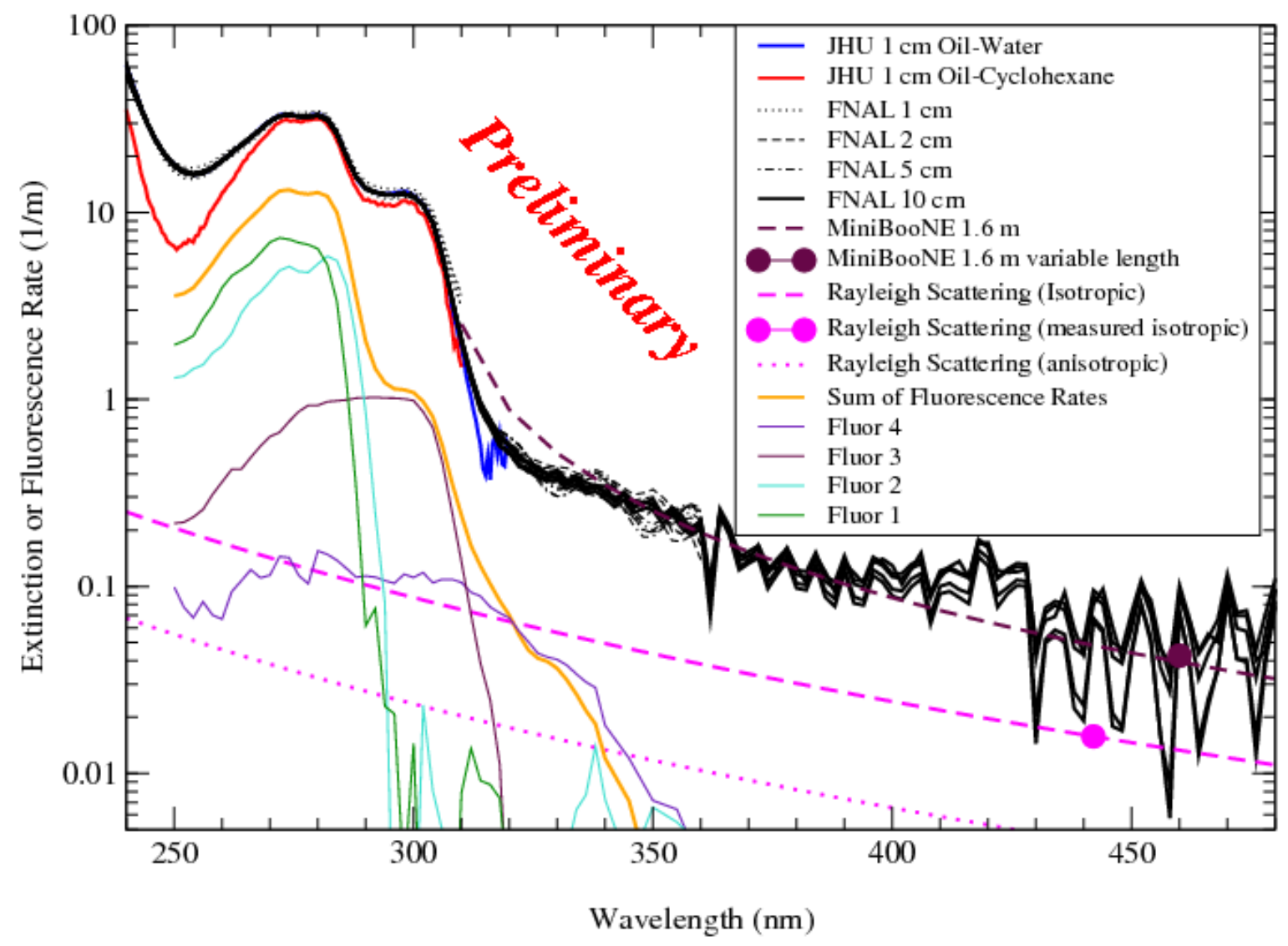

FIGURE 3.4. Photon attenuation rate in MiniBooNE mineral oil. Shown in this plot are the different components of the attenuation rate: scattering, absorption and fluorescence.

where $\mathrm{N}_{0}$ is the initial number of photons and $\lambda_{A}$ is the attenuation length. The wavelength dependence of the overall attenaution rate is measured by analyzing the transmission rate through samples of Marcol 7 oil using a spectrophotometer. The individual absorption, scattering, and fluorescence rates, together with the overall attenuation rate, are shown in figure 3.4 as a function of the photon wavelength. For a typical photon wavelength of $400 \mathrm{~nm}$, the attenuation rate is $7 \times 10^{-4} \mathrm{~cm}^{-1}$, corresponding to an attenuation length of $14 \mathrm{~m}$. For the same wavelength of 400 $\mathrm{nm}$, about half of the attenuation is due to absorption, and half due to scattering. 
For photons produced at $\lambda_{\gamma}=460 \mathrm{~nm}$ at the center of the MiniBooNE detector, approximately $19 \%$ are attenuated before reaching the PMT sphere.

\section{- Scattering}

Scattering is the deflection of optical photons due to interactions with molecules in the oil. The two main types of scattering seen in Marcol 7 are Rayleigh and Raman scattering. Rayleigh scattering is caused by thermal density fluctuations in the mineral oil that can cause light to scatter, changing the direction but not the wavelength of the incident photon. This process has a characteristic angular dependence for the scattered light of $\sim\left(1+\cos ^{2} \theta\right)$ for transverse polarization, and isotropic for longitudinal polarization.

In contrast, Raman scattering results in wavelengths that are red-shifted with respect to the initial photon wavelength because some of the photon's energy is lost to vibrational or rotational excitation. The probability of both Raman and Rayleigh scattering depends on wavelength as $\sim 1 / \lambda^{4}$, and both occur promptly in time. Isotropic Rayleigh scattering is the dominant contribution to the scattering process, although anisotropic Rayleigh scattering and Raman scattering are observed, contributing to roughly $20 \%$ and $7 \%$ of the total scattering rate, respectively.

\section{- Fluorescence}

Fluorescence is a related process that occurs when the molecular electron states are excited by optical photons instead of charged particles. The original photon is absorbed, hence the process is included in the absorption rate shown in figure 3.4 . As with scintillation light, the fluorescence light produced during the de-excitation of the target molecules is isotropic and delayed. The outgoing photons have longer 
wavelengths than the initial optical photons that excited the molecule. The fluorescent analysis of the Marcol 7 oil was performed by Dmitri Toptygin [56] from Johns Hopkins University. He identified four distinct fluors, each with its own emission spectrum, exctitation spectrum and emission time constant.

\section{- Absorption}

Photon absorption in the mineral oil is obtained from the difference between the attenuation rate curve and the sum of the fluorescence and scattering rates.

Photon attenuation in mineral oil, due to either fluorescent emission, scattering, or absorption, was measured at Fermilab with different experimental setups and over a wide photon wavelength range. The difference between the attenuation rate curve as a function of wavelength obtained by these measurements on the one hand, and the sum of the fluorescence and scattering rates discussed above on the other, is interpreted as photon absorption in mineral oil.

\subsubsection{Output of Detector Simulation}

The detector simulation gives the arrival time of all photons that hit the face of a PMT. The final step, a FORTRAN program called MCthroughDAQ, smears hit times and charges to determine their values at the PMT anode. The smearing functions were derived from ex situ measurements made with a MiniBooNE PMT. For each PMT, the program simulates the integration of charge by the DAQ electronics and fires a simulated discriminator if the charge threshold is crossed. Finally, MCthroughDAQ outputs simulated quads just like true detector data, so that both data and Monte Carlo may be treated in exactly the same way in reconstruction algorithms and the subsequent analysis of the events. 


\section{Chapter 4}

\section{Reconstruction Algorithm And Particle Identification}

\subsection{Event Reconstruction}

The reconstruction algorithm calculates the event vertex, direction, and energy using a maximum likelihood method based on the observed times and charges of all the hit PMTs. MiniBooNE uses two reconstruction software packages, the SFitter or Stancu fitter and the P-Fitter. Most of the analysis described in this thesis is based on the S-Fitter, and for that reason the focus will be on this reconstruction algorithm. The P-Fitter will be briefly discussed, but additional information can be found in [57].

\section{$4.2 \quad$ S-Fitter}

The S-Fitter was mostly written by Ion Stancu and the other members of the University of Alabama group. The fitter algorithm starts with the assumption that the light produced in the detector is due to a point-like source. The algorithm is structured so that it reconstructs the desired quantities in a step-by-step minimization approach, in which the complexity of the model prediction is gradually increased.

\subsubsection{Fast Fit: Time Likelihood Fit}

The first step of the reconstruction algorithm consists of maximizing a time likelihood function to determine the 4-vertex of an event. Given an event with vertex $\alpha\left(\mathrm{x}_{0}, \mathrm{y}_{0}, \mathrm{z}_{0}, \mathrm{t}_{0}\right)$ producing photons that hit a certain number $\mathrm{N}_{H}$ of PMTs in the tank, the probability for measuring a time $\mathrm{t}_{i}$ at the $\mathrm{i}^{\text {th }}$ PMT located at $\left(\mathrm{x}_{i}, \mathrm{y}_{i}, \mathrm{z}_{i}\right)$ is:

$$
P\left(t_{i} ; \alpha\left(x_{0}, y_{0}, z_{0}, t_{0}\right)\right)=\frac{1}{\sqrt{2 \pi} \sigma} \exp \left[-\frac{\left(t_{i}-t_{0}-r_{i} / c_{n}\right)^{2}}{2 \sigma^{2}}\right]
$$


where $t_{0}$ is the time at which the light was emitted, $\sigma \sim 1.8 \mathrm{~ns}$, is the time resolution of the PMTs, and

$$
r_{i}=\sqrt{\left(x_{i}-x_{0}\right)^{2}+\left(y_{i}-y_{0}\right)^{2}+\left(z_{i}-z_{0}\right)^{2}}
$$

is the distance from the $\mathrm{i}^{\text {th }}$ PMT to the light source. Also, $\mathrm{c}_{n}$ is the speed of light in the mineral oil and the quantity $r_{i} / c_{n}$ is used to correct for the light time of flight from the source point to the detection point. The likelihood to measure a set of times $\left(\mathrm{t}_{i}\right)_{i=1, N_{H}}, \mathrm{~N}_{H}<1280$ is therefore:

$$
\mathcal{L}\left(t_{i} ; \alpha\right)=\prod_{i=1}^{N_{H}} P\left(t_{i} ; \alpha\right) .
$$

It is important to recall that the likelihood function given in equation (4.3) was constructed with the assumption that the position of the light source was known, while the unknown variable was $t_{i}$, the time at which the light was detected. For MiniBooNE, the situation is rather the other way around. Typically, the position of the events producing light is unknown, but the detection time for a given PMT can be calculated using equation (2.1). Thus, for a measured set of time $\left(\mathrm{t}_{i}\right)_{i=1, N_{H}}$, the most likely event 4 -vertex is obtained by maximizing the likelihood function given in equation $(4.3)$ with respect to $\alpha[58,59]$. The starting value for the vertex in the maximization process is

$$
\overrightarrow{r_{0}}=\frac{1}{Q} \sum_{i=1}^{N_{H}} q_{i} \vec{r}_{i}
$$

the charge-averaged position, while the starting value for the time is chosen to agree with $\overrightarrow{r_{0}}$ [58]. The maximization of the likelihood function was performed using the fitting program MINUIT [60]. In equation (4.4), Q is the total charge summed over all the hit PMTS.

The maximization procedure yields a vertex position for each source of light, and it is used to estimate the hit time for each hit PMT. After correcting for the 
time of flight, hits with corrected time less than $\sim 4$ ns are considered prompt, and contained mostly $\check{C}$ erenkov light with a small fraction of scintillation light [58].

The vertex position is also used to estimate an event track direction. The direction of the track is extracted as the charge-averaged direction of the emitted light with respect to the maximized vertex. Finally, an approximate energy of the event is determined using the total charge and the distance of the fitted vertex from the optical barrier $[61,62]$.

\subsubsection{Full Fit}

The second step of the reconstruction algorithm uses as starting value the results from the StancuFastFit algorithm. The event timing and vertex are determined with better accuracy using a time and charge likelihood described below.

The charge likelihood function is a Poisson distribution, where the predicted charge $\mu_{i}$ at each PMT includes the effects of light attenuation, the PMT solid angle and PMT quantum efficiency. The probability to measure a set of $\mathrm{n}_{i}$ photoelectrons, given $\mathrm{N}_{H}$ PMTs, is the product of the charge likelihoods for each hit PMT:

$$
\mathcal{L}_{n}{ }^{i}\left(n_{i}, \mu_{i}\right)=\frac{1}{n_{i} !} \mu_{i}^{n_{i}} e^{\left[-\mu_{i}\right]}
$$

where the predicted average charge $\mu_{i}$ at each PMT is:

$$
\mu_{i}=\frac{\epsilon_{i}}{r_{i}{ }^{2}}\left[\Phi e^{-\frac{r_{i}}{\lambda_{S}}} f_{S}\left(\cos \eta_{i}\right)+\rho F\left(\cos \theta_{i}, E\right) e^{-\frac{r_{i}}{\lambda_{C}}} f_{C}\left(\cos \eta_{i}\right)\right]
$$

where $\epsilon_{i}$ is the PMT quantum efficiency, $\mathrm{r}_{i}$ is the distance from the vertex to the

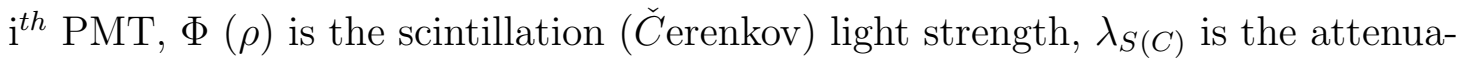
tion length for scintillation ( $\check{C}$ erenkov) light, $\mathrm{f}_{S(C)}\left(\cos \eta_{i}\right)$ is the PMT response to

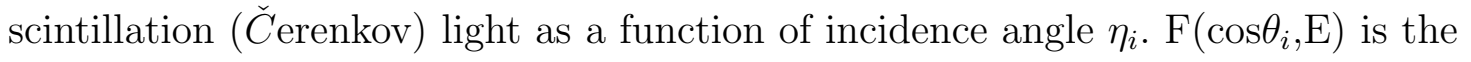
angular distribution of the $\check{C}$ erenkov light and is a function of the angle between the track and the line connecting the light source to the $\mathrm{i}^{\text {th }}$ hit PMT, and the track kinetic energy E. 
Taking into consideration the predicted charge for each PMT, the modified time likelihood is:

$$
\mathcal{L}_{t}{ }^{i}\left(t_{i}, \mu_{i}{ }^{C}, \mu_{i}^{S}\right)=\frac{\mu_{i}^{C}}{\mu_{i}^{C}+\mu_{i}^{S}} P_{C}\left(t_{i}, \mu_{i}{ }^{C}\right)+\frac{\mu_{i}^{C}}{\mu_{i}^{C}+\mu_{i}^{S}} P_{S}\left(t_{i}, \mu_{i}{ }^{S}\right)
$$

where $\mathrm{P}_{C}\left(t_{i}, \mu_{i}{ }^{C}\right)$ is the likelihood for $\check{C}$ erenkov light given in equation (4.1), and $\mathrm{P}_{S}\left(t_{i}, \mu_{i}{ }^{S}\right)$ is the likelihood for the scintillation light obtained by convolving equation (4.1) with an exponential decay. The event time and position are then obtained from maximizing the total likelihood function:

$$
\mathcal{L}_{T}=\prod_{i=1}^{N_{H}} \mathcal{L}_{q}{ }^{i}\left(n_{i}, \mu_{i}\right) \times \mathcal{L}_{t}{ }^{i}\left(t_{i}, \mu_{i}\right)
$$

where $\mathcal{L}_{q}{ }^{i}\left(n_{i}, \mu_{i}\right)$ and $\mathcal{L}_{t}{ }^{i}\left(t_{i}, \mu_{i}\right)$ are the probability of measuring a charge $q$ and a time $t$ at a given PMT. The vertex obtained from this step is called the mean gamma emission point (MGEP). Once the vertex is determined, an additional likelihood maximization is performed to determine the event direction.

\subsubsection{Flux Fit}

In this step, the MGEP, event time and direction are fixed while varying the scintillation and $\check{C}$ erenkov light strength $\Phi$ and $\rho$ in the above likelihood function

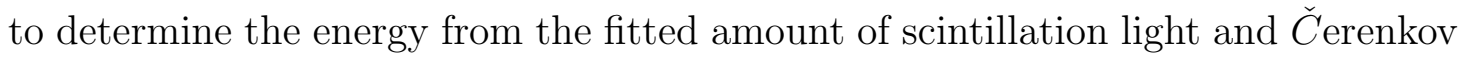
light.

\subsubsection{Track Reconstruction}

As mentioned earlier, in the reconstruction of electron-like events, the PMTs times and charges are assumed to be due to a point-like light source. For extended tracks like muons, the reconstruction assumes two identical light sources placed symmetrically around the middle point of the track, with each having half of the scintillation and $\check{C}$ erenkov strength computed in the earlier steps. The maximization algorithm 
determines the track length and a likelihood which is used to separate muons from electrons.

Neutral pions $\left(\pi^{0}\right)$ on the other hand are reconstructed using the two photons they produce when they decay. Since the MiniBooNE detector is unable to distinguish an electron from a $\gamma$, the fitter tries to identify photons by fitting for the photon conversion lengths. The model used for the fit consists of two electron tracks with shifted vertices.

\section{$4.3 \quad$ P-Fitter}

The P-Fitter was written by Ryan Patterson and the Princeton University group. The fitter starts with a specific profile for the emission of Čerenkov light and scintillation light which depends on the event energy. The expected charge on each PMT is then obtained from integrating the emission of light along the track convoluted with an acceptance function $\mathrm{J}(\mathrm{s})$ :

$$
\begin{array}{lll}
\mu_{c h} & \alpha \int_{-\infty}^{+\infty} \rho_{c h} J(s) F(\cos \theta(s) ; s) d s \\
\mu_{s c i} & \propto & \int_{-\infty}^{+\infty} \rho_{s c i} J(s) d s
\end{array}
$$

where $\mathrm{J}(\mathrm{s})$ is approximated with a parabola, and evaluated at three points along the track. $\rho_{c h}$ and $\rho_{s c i}$ are respectively the $\check{C}$ erenkov and scintillation light density functions. They reflect the fact that the light emissions are non-uniform along the track. $F(\cos \theta(s) ; s)$ is the angular emission profile for the $\check{C}$ erenkov light, which changes as the track propagates and loses energy.

The contribution of the $\check{C}$ erenkov light and scintillation light to the calculation of the time likelihood involves a complex procedure that is reported in detail in [57]. Except for this difference, the likelihood function in the P-Fitter, as in the S-Fitter, uses the mean emission profile to determine the event vertex position. 
Also, the charge likelihood function is calculated assuming a Poisson distribution for each hit as in the S-Fitter.

The P-Fitter relies on seven parameters including the $\check{C}$ erenkov strength, the scintillation strength and the track energy to determine whether the reconstructed single track is consistent with the electron hypothesis (short track) or the muon hypothesis (longer track). For the reconstruction of $\pi^{0}$ events, the single track algorithm is extended to include two photons originating from the pion decay. In total, twelve parameters are used to define $\pi^{0}$ events, and the fitting procedure is carefully designed to avoid cases where the negative of the log likelihood function is trapped in local minima.

The $\pi^{0}$ fit can also be run by setting a constraint on the invariant mass. In this case, the energy of the second photon is no longer a free parameter and is equal to:

$$
E_{2}=\frac{M_{\pi^{0}}^{2}}{2 E_{1}(1-\cos \gamma \gamma)}
$$

where $\cos _{\gamma \gamma}$ is the opening angle between the two photons. This fitting mode where the invariant mass is fixed is the standard $\pi^{0}$ hypothesis and its likelihood value is used a particle identification (PID) variable in chapter 6 .

With its light emission from extended tracks, the P-Fitter is better equipped for reconstructing muon tracks than the S-Fitter, and has better resolution in distinguishing $\pi^{0}$ events from electrons, although it runs much slower ( $\sim 10$ times). Both fitters reconstruct electrons with similar resolution.

\subsection{Particle Identification (PID)}

The goal of the particle identification algorithms is to find a set of characteristics that distinguish between particles such as muons, electrons and neutral pions. The intersection of the $\check{C}$ erenkov cone with the sphere of the PMTs produces ring 
profiles that are specific to each particle. Muons are characterized by single long tracks with ring profiles that are typically filled in. Electrons on the other hand are characterized by single shorter tracks, also producing a Čerenkov light ring, but because of multiple Coulomb scattering and Bremsstrahlung the rings are fuzzier than for muon tracks. The decay of a $\pi^{0}$ into $2 \gamma$ produces two rings, one for each photon conversion in the mineral oil. Figure 4.1 is a display of the ring profile for the different event types.

The PID algorithms discussed below are based on likelihood functions developed from reconstruction variables which emphasize the difference in the ring profiles produced by various particles.
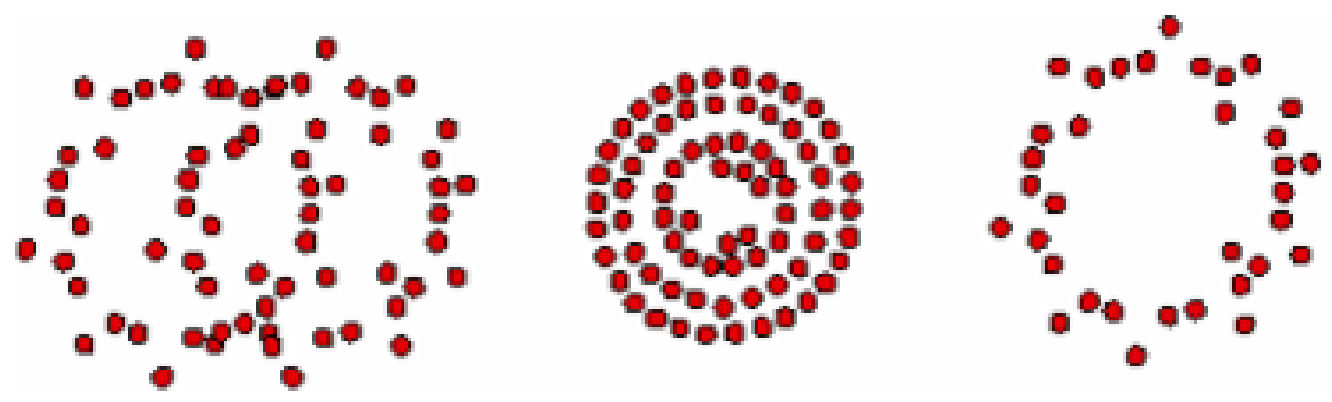

FIGURE 4.1. Hit topologies of electrons (left), muons (middle) and NC $\pi^{0}$ (right) events in the MiniBooNE detector.

\subsubsection{Track-Based Particle Identification}

The track-based PID algorithm is an extension of the P-Fitter [57]. With its ability to fit for electron, muon and pion track parameters, the fitter also produces likelihood variables $\mathrm{L}_{e}, \mathrm{~L}_{\mu}$ and $\mathrm{L}_{\pi}$ for each event type. The separation of electrons from muons, or electrons from pions, is achieved by setting a limit on the two PID variables: $\log \left(\mathrm{L}_{e} / \mathrm{L}_{\mu}\right)$ and $\log \left(\mathrm{L}_{e} / \mathrm{L}_{\pi}\right)$. In the left panel of figure 4.2 is an illustration of $\log \left(\mathrm{L}_{e} / \mathrm{L}_{\mu}\right)$ as a function of the reconstructed energy of the electron. The black curve on the plot shows the maximized cut value that separates electrons (blue) from muons (red). A similar plot is seen for $\log \left(\mathrm{L}_{e} / \mathrm{L}_{\pi}\right)$ in the right panel. 

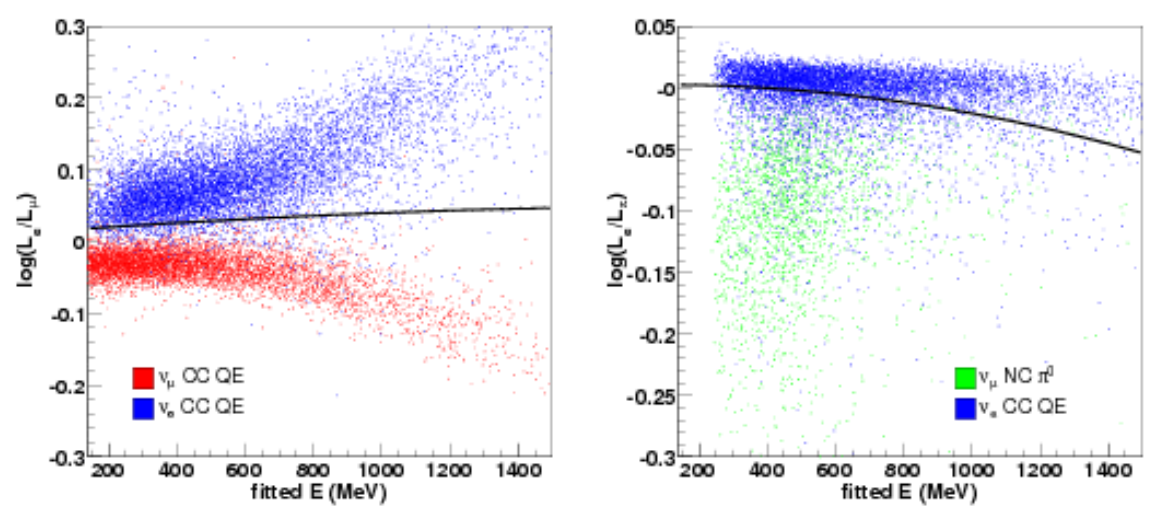

FIGURE 4.2. On the left, the distribution of $\log \left(\mathrm{L}_{e} / \mathrm{L}_{\mu}\right)$ as a function electron energy is used to separate a Monte Carlo simulation of $\nu_{e}$ CCQE events from a simulation of $\nu_{\mu}$ CCQE events. On the right, a simulation of $\nu_{e}$ CCQE events is separated from a simulation of $\nu_{\mu} \mathrm{NC} \pi^{0}$ events using the PID $\log \left(\mathrm{L}_{e} / \mathrm{L}_{\pi}\right)$ as a function of the electron energy. The values of the cuts were selected to optimized the oscillation sensitivity.

\subsubsection{Boosting Decision Tree}

The Boosting Decision Tree (BDT) algorithm for MiniBooNE [63] was developed to be used with the S-fitter. It combines 172 reconstructed variables to form a single output variable which will be applied in the following chapters to separate signal events (elastic scattering) from background events. The BDT is based on a binary classification tree that starts at the top node with Monte Carlo samples of pure signal events and pure background events. For each of the 172 variables, the algorithm tests different cut values to split signal events from the backgrounds. The variable and splitting value that gives the best separation is selected. In this step, the events have been split into two parts (branches), with one holding mostly signal events and the other mostly background events. For each branch, the process is repeated until a pre-selected final number of branches is obtained or each branch is pure signal or pure background.

To select the splitting variable and the splitting value at each node, a criterion based on a quantity called the Gini index is used. The splitting used is the one 


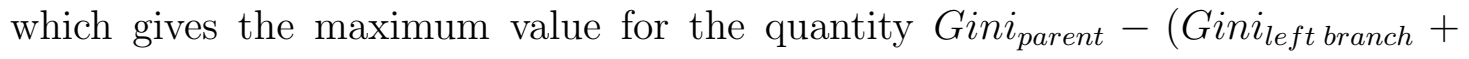
Gini $_{\text {right branch }}$ )

where the Gini index at a node is given by:

$$
\begin{array}{r}
\text { Gini }=P \times(1-P) \times N_{\text {tot }} \times \sum_{i=1}^{N_{\text {tot }}} w_{i} \\
\text { with }: P=N_{\text {signal }} / N_{\text {tot }}
\end{array}
$$

$\mathrm{N}_{\text {signal }}$ and $\mathrm{N}_{\text {tot }}$ are respectively the number of signal events and the total number of events at the node, $\mathrm{w}_{i}$ is the weight of event $\mathrm{i}$ and initially is set to $1 / \mathrm{N}_{\text {tot }}$. At the end of the process, if a node has purity greater than $1 / 2$, it is called a signal leaf and if the purity is less than $1 / 2$, it is a background leaf. Events are classified signal if they are on a signal leaf and background if they are on a background leaf.

If an event is misclassified, i.e, a signal event lands on a background leaf or a background event lands on a signal leaf, then the weight of the event is increased (boosted). A second tree is built using the new weights, no longer equal. Again misclassified events have their weights boosted and the procedure is repeated. Typically, one may build 1000 or 2000 trees this way. A score is now assigned to an event as follows. The event is followed through each tree in turn. If it lands on a signal leaf it is given a score of 1 and if it lands on a background leaf it is given a score of -1 . The renormalized sum of all the scores, possibly weighted, is the final score of the event. High scores mean the event is most likely signal and low scores that it is most likely background. By choosing a particular value of the score on which to cut, one can select a desired fraction of the signal or a desired ratio of signal to background.

The Track-based algorithm and the Boosting Decision Tree algorithm were both coded to identify $\nu_{\mu} \rightarrow \nu_{e}$ oscillation events. The latter was used as a check of the former and as reported in several papers including the MiniBooNE oscillation 
result [73], both algorithms showed similar results. Since the final state of high energy elastic scattering events is similar to $\nu_{e}$ CCQE, in chapter 6 we will use both algorithms to independently select high energy electron candidates. However, for low energy electrons other PID variables are required.

\subsubsection{PID Variables for Low-Energy $\nu_{\mu}$-e Events}

Most of the low energy events in the MiniBooNE detector are Michel electrons (electrons from the decay of both cosmic ray and beam related muons) and final state particles from neutral current elastic (NCEL) interactions. NCEL processes $\left(\nu_{\mu}+N \rightarrow \nu_{\mu}+N\right)$ are interactions where neutrino scatters off the nucleus, or a nucleon with a small energy transfer. They produce mostly scintillation light because the final state nucleons are below $\check{C}$ erenkov threshold. These processes are described in [64], and are an important source of background in the analysis of low energy elastic scattering of neutrinos on electrons.

To separate $\nu_{\mu}$ e events from Michel electrons, we constructed a PID that identifies a Michel electron by tagging it to the parent muon it decayed from. The details of this procedure are the subject of section 5.3 .1 of chapter 5 .

The separation of low energy electrons from nucelons produced in NCEL processes is achieved with a variable based on time likelihood that was initially designed to reject electrons for an analysis focused on NCEL events [64]. Here, the same variable is used, just the other way around. More information on this variable is given in section 5.4 .1 of chapter 5 . 


\section{Chapter 5}

\section{Low Energy Elastic Scattering Events}

To measure the muon neutrino magnetic moment, we analyzed the interactions $\nu_{\mu}+\mathrm{e} \rightarrow \nu_{\mu}+\mathrm{e}$, looking for an excess of low energy elastic scattering events. The excess could be interpreted as an effect of the electomagnetic interaction because the cross section due to magnetic scattering

$$
\left(\frac{d \sigma}{d T}\right)^{E M}=\pi r_{0}^{2} f^{2}\left(\frac{1}{T}-\frac{1}{E_{\nu}}\right)
$$

becomes larger at low $\mathrm{T}$ than the cross section of the weak interaction

$$
\left(\frac{d \sigma}{d T}\right)^{\text {weak }}=\frac{2 m_{e} G_{F}^{2}}{\pi}\left[g_{L}^{2}+g_{R}^{2}\left(1-\frac{T}{E_{\nu}}\right)^{2}-g_{L} g_{R} \frac{m_{e} T}{E_{\nu}^{2}}\right]
$$

In (5.1) and (5.2),

- $\mathrm{G}_{F}$ is the Fermi coupling constant.

- $\mathrm{g}_{L}=-1+2 \sin ^{2} \theta_{W}$

- $\mathrm{g}_{R}=2 \sin ^{2} \theta_{W}$

- $\theta_{W}$ is called the Weinberg angle and is a fundamental parameter of the G-S-W model discussed in chapter 1 .

- $\mathrm{T}$ is the kinetic energy of the recoil electron.

- $\mathrm{E}_{\nu}$ is the energy of the incident neutrino.

- $\mathrm{r}_{0}$ is the classical electron radius $\left(\mathrm{r}_{0}=2.8210^{-13} \mathrm{~cm}\right)$.

- $\mathrm{f}$ is the ratio of the neutrino magnetic moment to the Bohr magneton. 
The signature for $\nu_{\mu} e$ events is determined by equation (5.3) which shows elastically scattered electrons are produced at very forward angles.

$$
\cos \theta=\frac{1+\frac{m e}{E_{\nu}}}{\sqrt{1+\frac{2 m_{e}}{T}}}
$$

\subsection{Overview of the Event Samples}

At MiniBooNE, an event is defined as a $19.2 \mu$ sec-long time window that opens coincident with the arrival of the neutrino beam. This time window is divided into subevents, a collection of PMT hits clustered in time within 100 ns.

\subsubsection{Data Sample}

The data for this analysis were collected after the fall of 2003 because prior to that date, information necessary to study the background due to cosmic ray muons was not available. The corresponding number of protons-on-target is therefore 4.67E20, lower than the number for the oscillation analysis (5.58E20) [31]. The study of the background due to cosmic ray muons is presented in section 5.3.

\subsubsection{Simulated Beam-Induced Events}

For the purpose of constraining the background and understanding the kinematics of the signal events, different event samples were used. Background events induced by the neutrino beam were studied with a sample of 9.1 million Monte Carlo beam events, corresponding to 2.28E21 protons-on-target. These events are generated uniformly in a volume with a radius of 12 meters. Thus, the sample includes background events arising from the neutrino interactions in the dirt surrounding the detector, as well as neutrino interactions in the tank. Also, 305000 electroweak $\nu_{\mu} \mathrm{e}$ events, the equivalent of 1.74E24 protons-on-target, were generated to study the acceptance of our selection cuts. 


\subsubsection{Beam Off Events}

The beam unrelated activity mostly originates from cosmic ray muons, electrons from muon decay (Michel electrons), and PMT dark noise. The level of the beam unrelated background is measured using strobe events that are obtained with a random trigger that pulses at a frequency of $2.01 \mathrm{~Hz}$ between beam spills. In that sense, the strobe trigger is identical to the beam trigger except that it does not contain any beam neutrino events.

Over 5 million strobe events were used to study the largest beam-off background for the elastic scattering analysis which is due to Michel electrons from cosmic ray muons that stop in the tank and decay.

\subsection{Low Energy $\nu_{\mu}$ e Event Selection}

The selection of low energy elastic scattering events from the beam data starts with a set of precuts to identify potential low energy electrons in the forward direction. The precuts are:

- only one subevent:

Since we expect to observe only the electron from the $\nu_{\mu} e \rightarrow \nu_{\mu} e$ interaction, a one subevent criteria was imposed to reject electrons from muon decay when the electron and muon both produce subevents in the beam window.

- $\cos \theta>0.90$ :

This cut is motivated by equation (5.3) which shows that $\nu_{\mu} e$ events are strongly peaked at low angle.

- $4550 \mathrm{~ns}<$ subevent time $<6250$ ns:

The subevent time cut is based on the observed time distribution for the CCQE process as displayed in 5.1, where it is observed that most beam related interactions occur between $4550 \mathrm{~ns}$ and $6250 \mathrm{~ns}$. Using as narrow a 
time window as possible helps reduce the rate of cosmic ray events in the data sample.

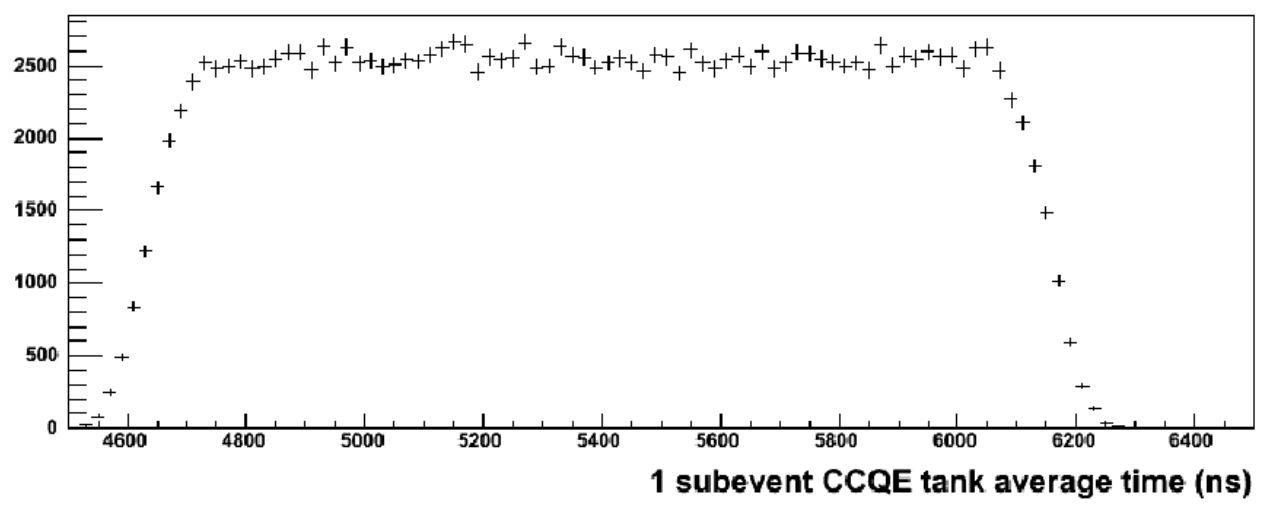

FIGURE 5.1. The time distribution of the CCQE first subevent is shown to be between 4550 and $6250 \mathrm{~ns}$. We expect this situation to be identical for $\nu_{\mu} e$ events.

- $15 \mathrm{MeV}<$ electron energy $(\mathrm{T})<100 \mathrm{MeV}$ :

Most muons that stop in the oil decay but $7.8 \pm 0.2 \%$ of $\mu^{-}$are captured, and $18 \%$ of the captures produce boron nuclei that beta decay: ${ }^{12} \mathrm{~B} \rightarrow{ }^{12} \mathrm{C}$ $+\mathrm{e}^{-}+\overline{\nu_{e}}$ with an energy endpoint at $13.4 \mathrm{MeV}$. With no specific means of distinguishing these electrons from the $\nu_{\mu}$ e events, an energy threshold at 15 $\mathrm{MeV}$ is required to avoid a contamination of the data with electrons from the decay of ${ }^{12} \mathrm{~B}$.

The motivation for $\mathrm{T}<100 \mathrm{MeV}$ is because above $100 \mathrm{MeV}$ the electromagnetic contribution to the cross section is small compared to the weak cross section for any magnetic moment allowed by current experimental limits. Therefore the search for the neutrino magnetic moment will be conducted in the energy region below the $100 \mathrm{MeV}$ limit.

- $\mathrm{N}_{\text {veto }}<6$

This is the standard veto cut used in MiniBooNE to reject particles entering or leaving the detector. 
- Radius $<500 \mathrm{~cm}$

This is the usual fiducial cut used in MiniBooNE to ensure that events are contained in the detector, within the region where they can be accurately reconstructed.

- $20<\mathrm{N}_{\text {tank }}<400$

Low energy events with an anomalously high number of PMTs hits are removed by requiring the number of hit PMTs in the tank be less than 400. 20 hit PMTs are required to reconstruct events with an acceptable resolution.

\subsection{Study of the Cosmic Ray Background}

A significant fraction of the events that pass the precuts are Michel electrons with their parent muons preceeding the beam window. As shown in figure 5.2, the time distribution of events passing all the precuts, except for the beam time cut, falls off exponentially with a lifetime of $\sim 2 \mu$ sec, indicating that the events are mostly Michel electrons.

The strobe sample described in 5.1 .3 is used to develop a set of criteria to help reject Michel electrons by identifying the muons they decayed from. The criteria are based on a trigger level [32] that measures the tank activity prior to the presence of an event. The trigger level provides us with the time of an activity along with the number of hit PMTs in both the tank and the veto region. From this information and using the time the beam window opens (Time Origin) we will construct a variable called Past Time.

\subsubsection{Description of The Past Time Cut}

The identification of a Michel electron consists of looking for a time correlation between the subevent in the beam window and a tank activity prior to the time the window opens. The search for the muon uses the past activity trigger level 


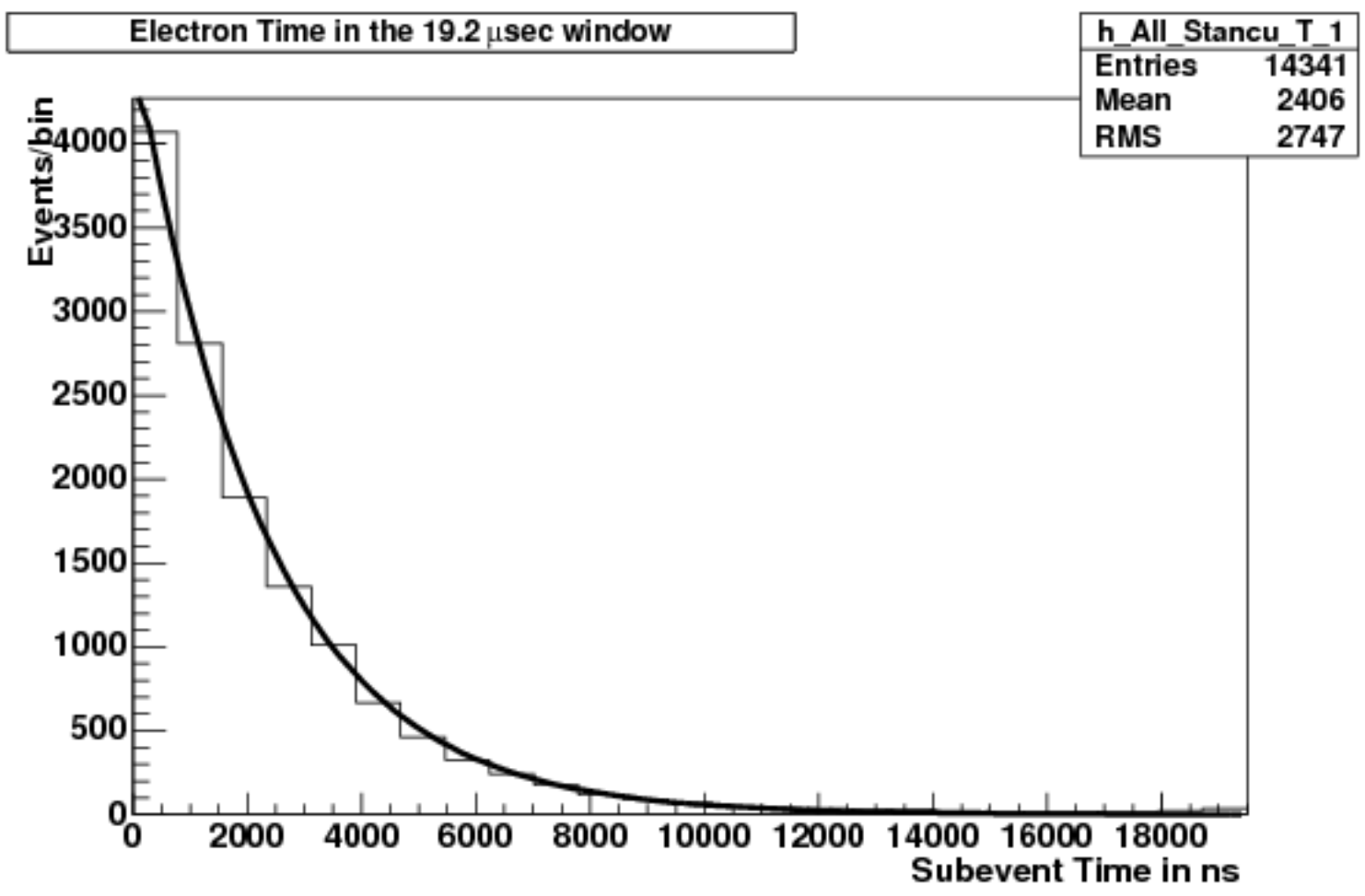

FIGURE 5.2. This plot shows the time distribution of events passing all the precuts except the beam time cut. The exponential fall off indicates most of these are Michel electrons with their parent muon outside the window.

that requires 60 tank hits (DET5). The main reason for this choice is because of its stability compared to other past time trigger levels, and also because requiring 60 tank hits is a high enough threshold to eliminate noise events while almost all low energy muons are still detected. For an activity that satisified this trigger criterion, the Past Time is constructed from the activity time, provided by a GPS clock, by substracting the Time Origin of the beam window. If there is a correlation between the subevent and the activity detected before the window opened, the Past Time will exhibit a specific distribution that underlines that correlation. Initially, an offset value of $0.5 \mu \mathrm{sec}$ was subtracted from the past event time to take into account electronic effects [65], but based on analysis of the data, we subtracted 
Exponential fit of the past time distribution

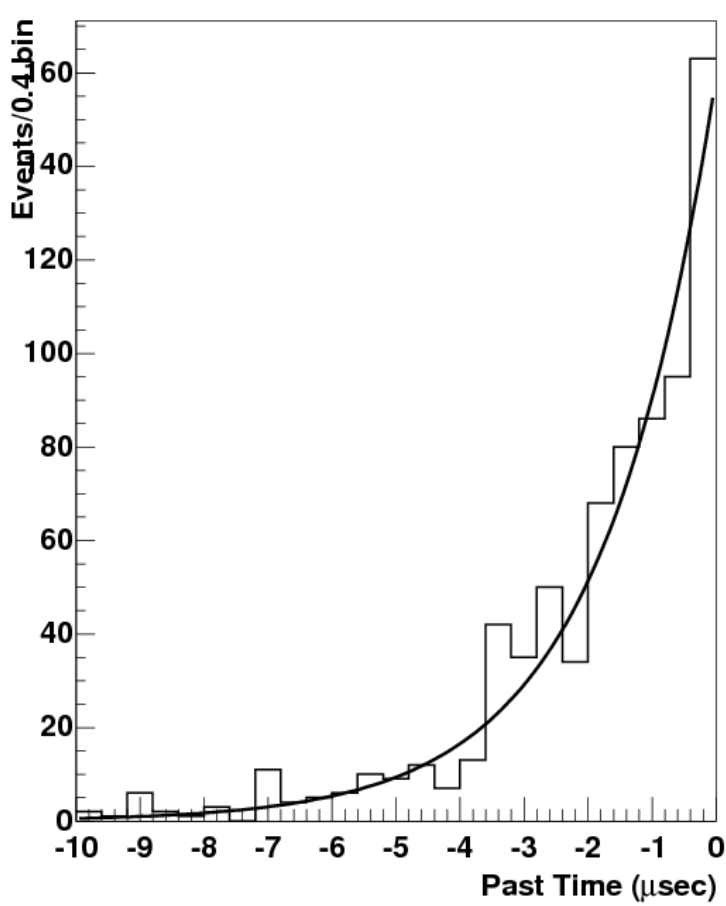

Energy of Michel Events

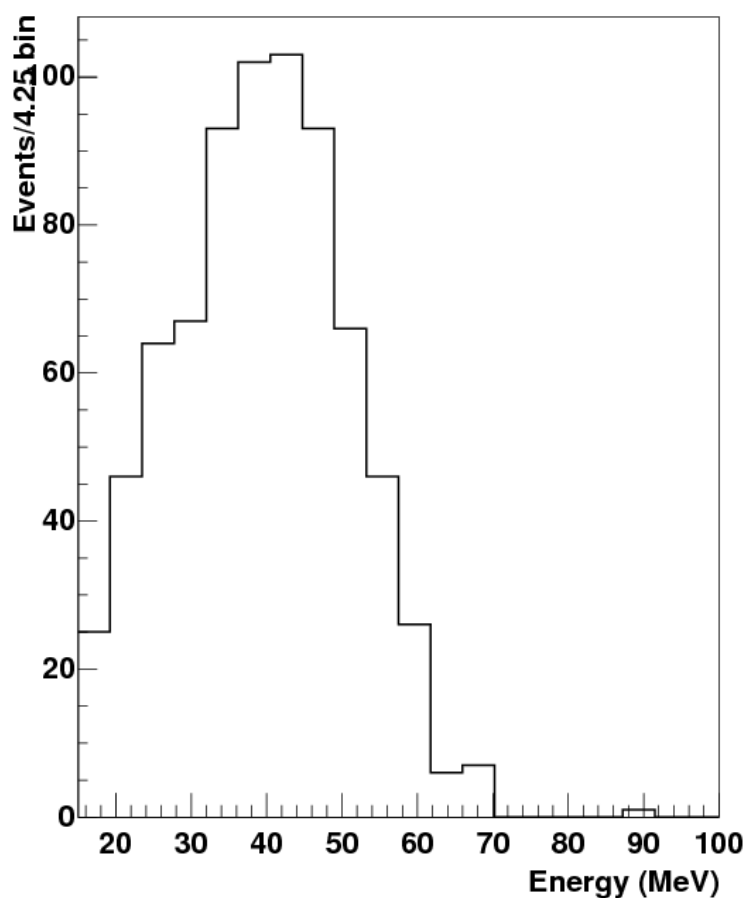

FIGURE 5.3. On the left is shown the past event time distribution for strobe events passing the precuts. Fitting the distribution to an exponential yields a decay lifetime of $1.87 \pm 0.3 \mu \mathrm{sec}$, consistent with the muon lifetime. The energy distribution of subevents with activity in the $10 \mu$ sec interval before the window opens is displayed on the right, and is consistent with the energy distribution of Michel events.

an additional $0.5 \mu$ sec to take into account the fact that high energy cosmic ray muons often push the Past Time past the Time Origin.

The Past Time, measured in microseconds, is then given by:

$$
T_{\text {past }}=T_{\text {event }}-T_{\text {Origin }}-1
$$

where $T_{\text {event }}$ is the time of the past activity measured by the GPS clock.

In figure 5.3, the left plot is the past time distribution of strobe events satisfying the precuts. An exponential fit of the distribution between $-10 \mu$ sec and 0 yields a lifetime of $1.87 \pm 0.3 \mu$ sec consistent with the hypothesis that the observed events are electrons that have decayed from muons detected before the beam window 
opened. The plot on the right shows the energy distribution of these events. It is consistent with the energy distribution expected for Michel electrons.

The strobe study indicated that requiring the past time to be greater than $10 \mu$ sec removes $96 \%$ of the Michel electrons from cosmic ray muons for events satisfying the precuts.

subsectionCosmic Background After Past Time Cut

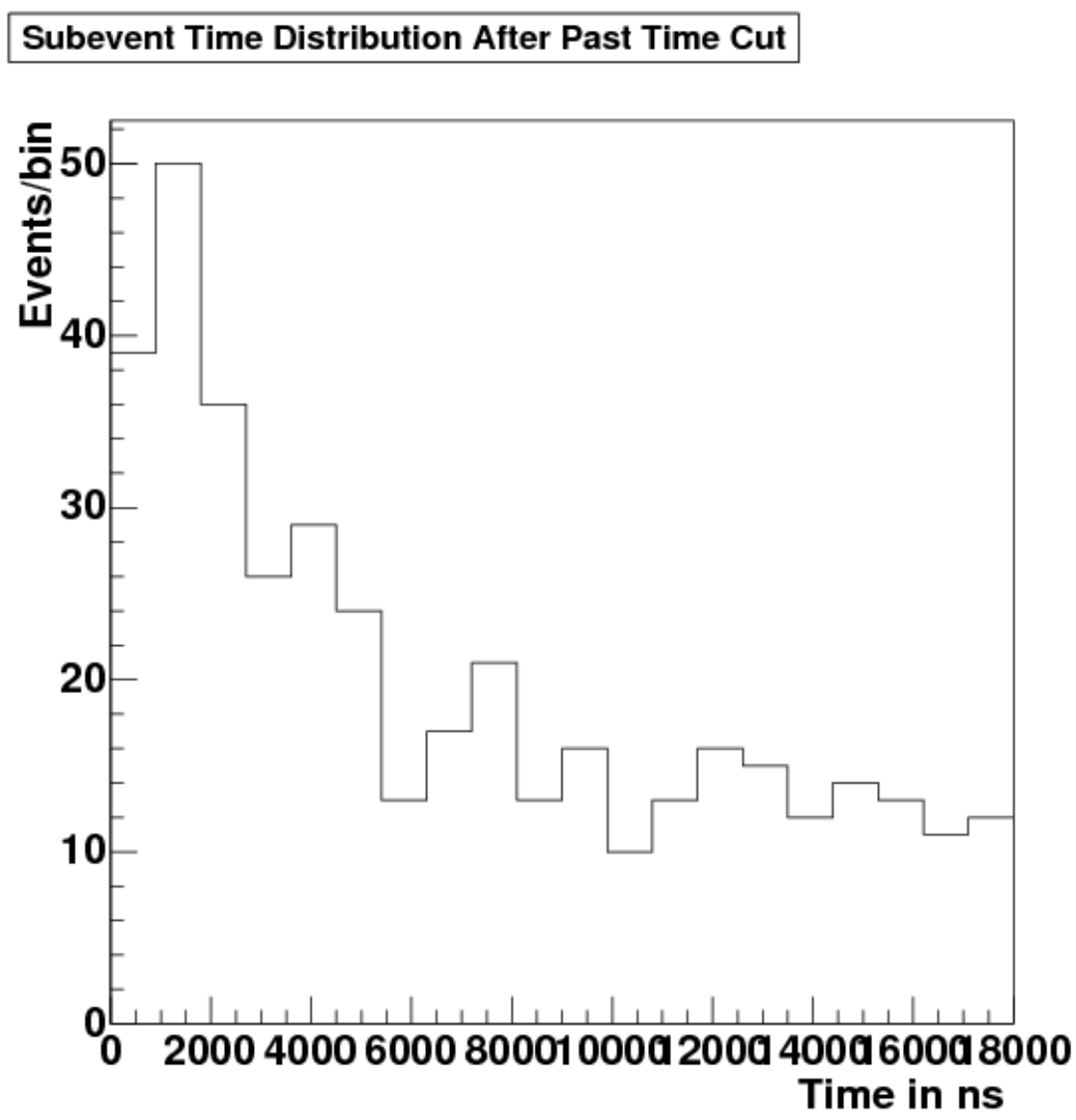

FIGURE 5.4. This plot is a display of the $\Delta \mathrm{t}$ distribution for subevents for which we failed to find an activity in the tank $10 \mu$ sec before the beam window opened.

Beyond $10 \mu$ sec, any coincidence between the two events is mostly accidental. The remaining background events are largely due to Michel electrons for which we failed to detect the parent muon or from remnants of the interactions of cosmic ray 
particles with the material in and around the detector. In figure 5.2, it was obseved that the number of events satisfying the one subevent cut decreases exponetially with time as expected from the muon lifetime, indicating that the majority are electrons due to the decay of cosmic muons. We now consider a new variable $\Delta \mathrm{t}$ which is the sum of the event time and the past time minus $14 \mu \mathrm{sec}$,

$$
\Delta t=\left|T_{\text {past }}\right|+T_{\text {subevent }}-14 \mu s e c
$$

In terms of background rejection versus signal loss it is better to cut on $\Delta \mathrm{t}$ than it is to simply make a tighter cut on past time. Figure 5.4 shows the distribution of events in the strobe sample passing the precuts and the 10 microsecond past time cut. Based on this distribution, and the signal acceptance, a cut of 3 microseconds was imposed on $\Delta \mathrm{t}$.

The efficiency of the time cuts is obtained by applying the cuts on an event sample free of Michel electrons. For this purpose, we used high energy data events and required that the number of hit PMTs in the tank be greater than 200. This cut was chosen because the Michel energy endpoint is $53.8 \mathrm{Mev}$ corresponding to about 175 hit PMTs in the tank. $82.0 \pm 0.3 \%$ of these data events pass the time cuts, meaning that the probability of misidentifying a random coincidence of two events as the decay of a cosmic muon into a Michel is $18.0 \%$.

\subsection{The Beam Related Background}

The beam related non- $\nu_{\mu}$ e events surviving the precuts are composed of In-tank events (events that originate within the tank) and events produced outside the tank from the interaction of neutrinos mostly with the upstream dirt and other material surrounding the detector. These events are refered to as dirt events. As was discussed in chapter 4, the Monte Carlo simulation of $\nu_{\mu}$ e events in the NUANCE event generator is based only on the weak interaction. For this resason, 
we will often refer to the $\nu_{\mu} \mathrm{e}$ events as the electroweak events, and the non- $\nu_{\mu} \mathrm{e}$ events (dirt and In-tank) as the beam related background.

\subsubsection{In-tank Events}

\section{Electron PID}

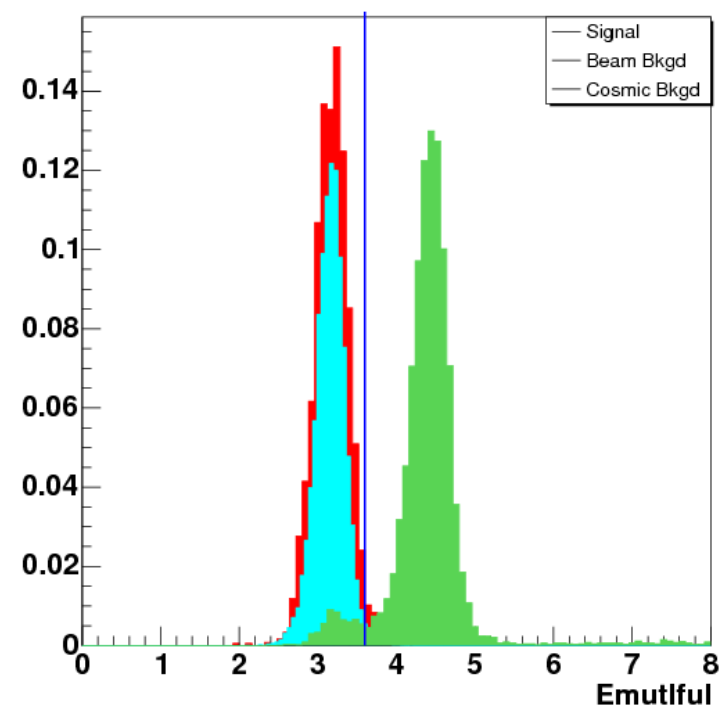

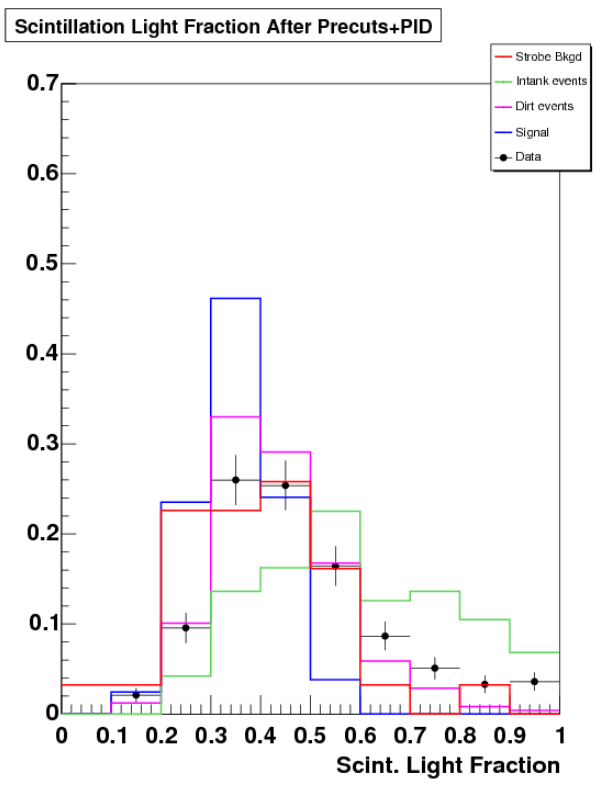

FIGURE 5.5. Left: The PID variable shows a good separation between electron candidates (blue and red) from beam related background (green). The cyan histogram is a Monte Carlo simulation of $\nu_{\mu}$ e events, while the red histogram shows electrons from cosmic events that we remove with the past time cut. Events in this plot have passed the precuts requirement. Right: The scintillation light fraction distribution for dirt (magenta), electroweak (blue), NCEL (green) and cosmic (red) events are shown. In this plot, the events were required to pass both the precuts and the PID cut.

At low energy, most of the In-tank events are particles below $\breve{C}$ erenkov threshold that mimic electrons. $73 \%$ of these events are the product of neutral current elastic processes $\nu_{\mu} N \rightarrow \nu_{\mu} N$ (NCEL) which produce a recoil nucleon below $\check{C}$ erenkov threshold while the neutrino leaves the detector with no trace. As described in section 4.4.3, an electron PID variable based on PMT time likelihhood is used to separate electrons from NCEL events. The variable is shown on the left side of figure 5.5, and a cut at 3.6 eliminates $98 \%$ of the In-tank background. 
Also, since NCEL processes typically produce scintillation light, the fraction of scintillation light can be used to further decrease the NCEL background. The right side of figure 5.5 shows the distribution of the scintillation light fraction for the elastic scattering events (blue), NCEL events (green), cosmic background events (red), and dirt events (magenta) after the precuts and the PID variable cut requirements. Requiring that the scintillation light fraction (LF) be less than 0.55 decreases the remaining NCEL events by $\sim 40 \%$, the dirt and the cosmic events by $\sim 20 \%$ and the elastic scattering events by less than $\sim 1 \%$.

\subsubsection{Dirt Events}

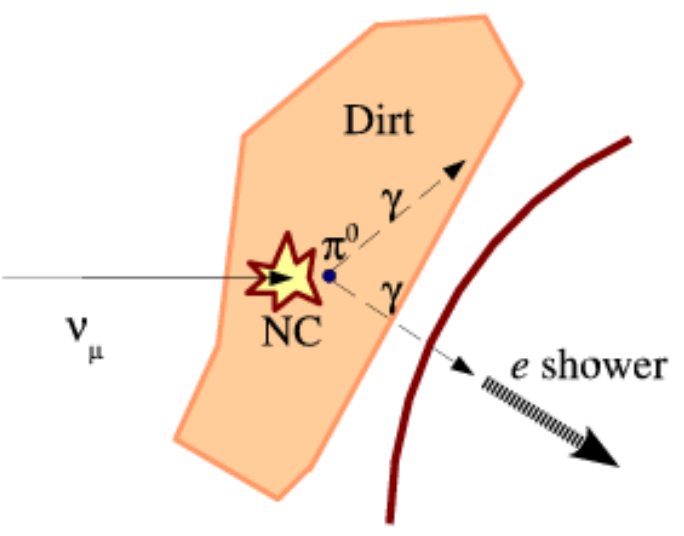

FIGURE 5.6. A cartoon representation of a photon from the decay of a $\pi^{0}$ event produced from the interaction of neutrinos with the dirt outside the detector.

Photons produced in the dirt events described in section 5.4 can penetrate the veto region and find their way into the main tank. They constitute an important source of background because they could easily be misidentified as low energy electrons. Fig. 5.6 is a cartoon representation of an interaction in which a single $\gamma$ arising from $\pi^{0}$ decay enters the detector. To measure the rate of dirt events in the data, we used a Monte Carlo sample of events generated outside the tank and applied a cut on a reconstructed quantity that measures the distance of the event 
vertex to the wall of the tank in the backward direction. This quantity often called Rtowall is defined as:

$$
\text { Rtowall }=\vec{R}_{\text {event }} \cdot \vec{U}_{\text {event }}+\left[\left(\vec{R}_{\text {event }} \cdot \vec{U}_{\text {event }}\right)^{2}-\left|\vec{R}_{\text {event }}\right|^{2}+\left|R_{0}\right|^{2}\right]^{1 / 2}
$$

where $\vec{R}_{\text {event }}$ represents the reconstructed event-vertex, while $\vec{U}_{\text {event }}$ is the reconstructed direction cosines. Here $\mathrm{R}_{0}$ is the radius of the fiducial sphere which was set to $500 \mathrm{~cm}$ as explained in the precuts from the event selection in section 5.1. It is important to note that most MiniBooNE analyses that use the rtowall variable have used $\mathrm{R}_{0}=550 \mathrm{~cm}$, which is the distance from the center of the tank to the surface of the PMTs.

As can be seen in figure 5.7, a cut at $210 \mathrm{~cm}$ on the Rtowall removes $70 \%$ of the generated dirt events, but only $22 \%$ of the non-dirt.

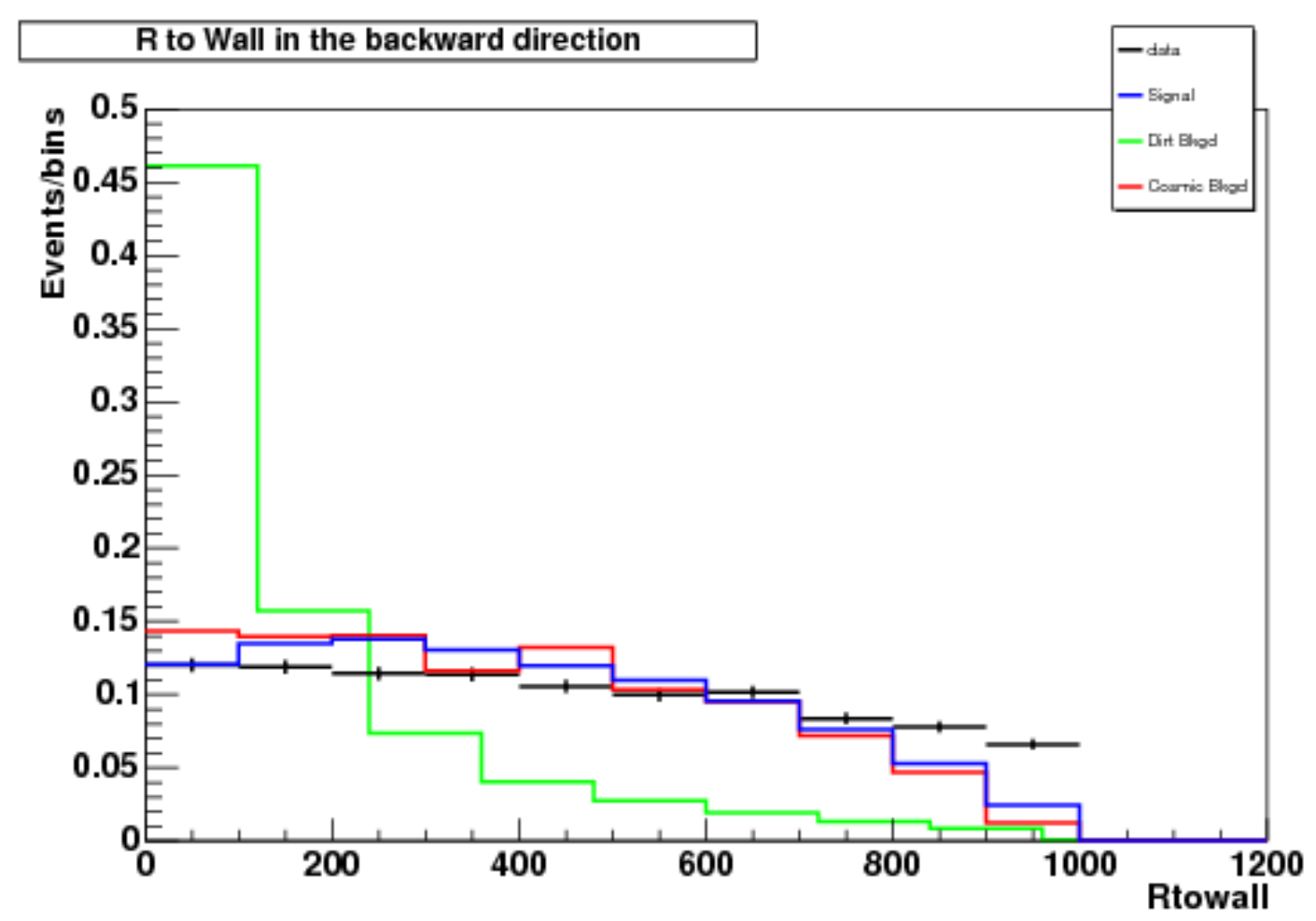

FIGURE 5.7. Rtowall distribution for events passing the precuts. 
After the Rtowall cut, an inspection of the veto hits distribution, figure 5.8, indicates that most low energy electrons fire fewer than two PMTs in the veto region. The plot shows that over $97 \%$ of the events in various electron samples (Michel electrons from $\mathrm{CC} \pi^{+}$in light green, electroweak electrons in blue and Cosmic background events in red ) are below two PMT hits while only $72 \%$ of the remaining dirt (magenta) events are below the two PMT mark. A Vhits $<2$ cut will therefore eliminate a significant number of the remaining dirt events with a marginal loss of sensitivity.

Veto Hits Distribution after Precuts+Rtowall

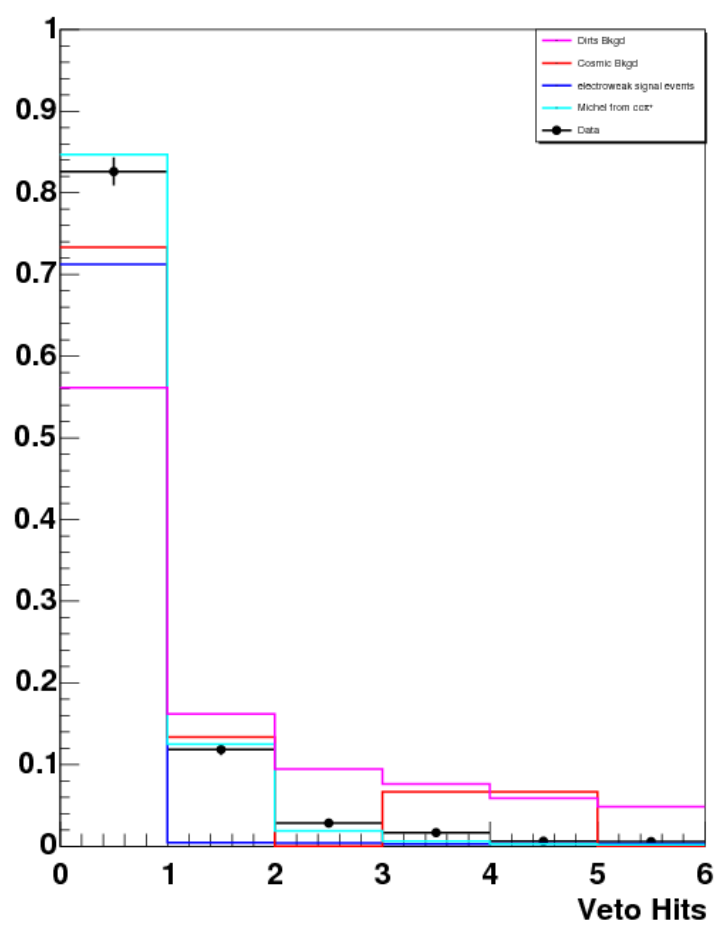

FIGURE 5.8. The veto hits distributions for data events (black points) and different Monte Carlo event samples are shown. Dirt events are in magenta, cosmic events in red, electroweak events are in blue and Michel electrons from $\mathrm{CC} \pi^{+}$are in light green. 
A table summarizing the result of all the cuts used on the events that passed the precut selection is shown below. Also shown are the motivation behind the cuts and the cut effectiveness.

TABLE 5.1. A summary of all the cuts used after the precuts listed in section 5.2. The motivation behind each cut and the effectiveness of the cut for each data sample are given.

\begin{tabular}{|c|l|l|}
\hline & Motivation & Effectiveness \\
\hline Past time $<10 \mu \mathrm{sec}$ & Cosmic events & Remove 94.5\% \\
\hline$\Delta \mathrm{T}>3 \mu \mathrm{sec}$ & Cosmic events & Remove an additional 2\% \\
\hline $\mathrm{PID}<3.6$ & In-tank events (mostly NCEL) & Remove 98\% \\
\hline LF $<0.55$ & In-tank, dirt, cosmic events & Remove an additional 1\% of NCEL \\
\hline Rtowall $<210 \mathrm{~cm}$ & Dirt events & Remove $70 \%$ \\
\hline Vhits $<2$ & Dirt events & Remove an additional 18\% \\
\hline
\end{tabular}

\subsection{Final Event Sample And Normalization of the Background: Counting Method}

Table 5.2 shows the event samples after each cut was applied sequentially. Note that the time cuts are not applied to the Monte Carlo sample as it contains no cosmic ray events. The normalization of the events from Monte Carlo samples was

TABLE 5.2. This table shows the observed number of events after each cut is applied sequentially.

\begin{tabular}{|c|r|r|r|r|r|r|r|}
\hline & PreCuts & Past T. & $\Delta \mathrm{T}$ & PID & LF & Rtowall. & Vhits \\
\hline Cosmic Bkgd & 904 & 63 & 62 & 32 & 28 & 8 & 7 \\
\hline In-tank Bkgd & 22784 & N/A & N/A & 198 & 89 & 44 & 39 \\
\hline Dirt Bkgd & 4034 & N/A & N/A & 1336 & 1115 & 87 & 66 \\
\hline Signal Events & 24201 & N/A & N/A & 23892 & 23180 & 16846 & 16575 \\
\hline Data & 5244 & 3629 & 3589 & 277 & 222 & 63 & 51 \\
\hline
\end{tabular}

obtained using the NUANCE predicted event rate for 4.67E20 protons-on-target, and the results are shown in table 5.5. The procedure consisting of normalizing 
the expected number of beam events to the data POT and comparing the result to the measured number of data events is called the counting method.

The normalization of the cosmic ray events which must also be taken into consideration in the counting method, is based on the Michel electrons rejected by the past time cuts. The rate of cosmic ray Michel electrons should be the same for the strobe and data sample. Using the strobe data that pass the precuts, the ratio of the number of events that survive the past time cuts to the number removed by the cuts is: $62 /(904-62)$. Assuming that the strobe events removed by the time cuts are Michel electrons and using the $82 \%$ past time cuts efficiency, it can be shown that the predicted cosmic ray related background after the precuts and the time cuts in the data is:

$$
N_{\text {cosmic }}^{\text {after precuts }}=(5244-(3589 / 0.82)) \times \frac{62}{(904-(62 / 0.82))} \sim 65.0 \text { events }
$$

The numbers in equation (5.7) are obtained from table 5.2. After the remaining cuts are applied, the strobe sample shows that only $\sim 11 \%$ of the cosmic related background will survive. Thus, the expected number of cosmic related background in the data sample after all the cuts are applied is:

$$
N_{\text {cosmic }}^{\text {after all cuts }}=0.11 \times 65.0 \sim 7.2 \text { events } .
$$

\subsection{Observation of An Excess of Low Energy Events in the Forward Direction}

From table 5.5, the expected total number of beam and cosmic ray events is lower than the observed data events. Table 5.6 shows the number of data events, and the expected number of electroweak events and background events for 4.67E20 pot. We will discuss the significance of the excess in the number of data events in section 5.10 after presenting a method of obtaining the excess more directly from 
TABLE 5.3. A summary of the different event samples used for the analysis is presented. The second column shows the number of events that have passed the precuts. The third column is the number of events after all cuts were applied, while the next column shows the corresponding protons-on-target for each sample. Column 5 displays the expected rate for the $\nu$ e elastic scattering plus all the significant backgrounds together with the observed number of data event all corresponding to 4.67E20 pot.

\begin{tabular}{|c|r|r|r|r|}
\hline \hline & $\mathrm{N}_{\text {events }}^{\text {After precuts }}$ & $\mathrm{N}_{\text {events }}^{\text {After All Cuts }}$ & Sample POT & $\mathrm{N}_{\text {events }}^{\text {Normalized }}$ \\
\hline Beam Bkgd & 26818 & 105 & $2.28 \mathrm{E} 21$ & 21 \\
\hline Cosmic Bkgd & 904 & 11 & N/A & 7.2 \\
\hline$\nu_{\mu}$ e events & 24201 & 16575 & $1.74 \mathrm{E} 24$ & 4.6 \\
\hline Data & 5244 & 51 & $4.67 \mathrm{E} 20$ & 51 \\
\hline
\end{tabular}

TABLE 5.4. A table comparing the data event rate to the expected event rate. We observe an excess of 18.2 events above the expected signal and background.

\begin{tabular}{|c|r|r|r|}
\hline Data & Bkgd & $\nu_{\mu} \mathrm{e}$ & Excess \\
\hline 51 & 28.2 & 4.6 & 18.2 \\
\hline
\end{tabular}

the data. Figure 5.9 shows data and background events as function of the angular distribution and the recoil electron energy. The Monte Carlo prediction includes the electroweak events in blue and the beam background in green. The red curve shows the expected background from cosmic events. The excess is also observed in the radius distribution and the electron time distribution as shown in figure 5.10, as well as in the distributions of the electron spatial coordinates as shown in figure 5.11. The same observations can be made for the distributions shown in figure 5.12 which include the distribution of $\vec{U} \cdot \vec{R}$, a variable used in calculating rtowall. It is important to note that the observed excess in the radius distribution is much bigger at large $\mathrm{R}$, indicating that there may be a background not well simulated.

\subsection{Extracting Signal From $\operatorname{Cos} \theta$ Distribution}

The number of elastic scattering events can also be obtained directly from the data by minimizing a negative log-likelihood function constructed on the assumption that the data sample with $\cos \theta>0.90$ is composed of elastic scattering electrons and 

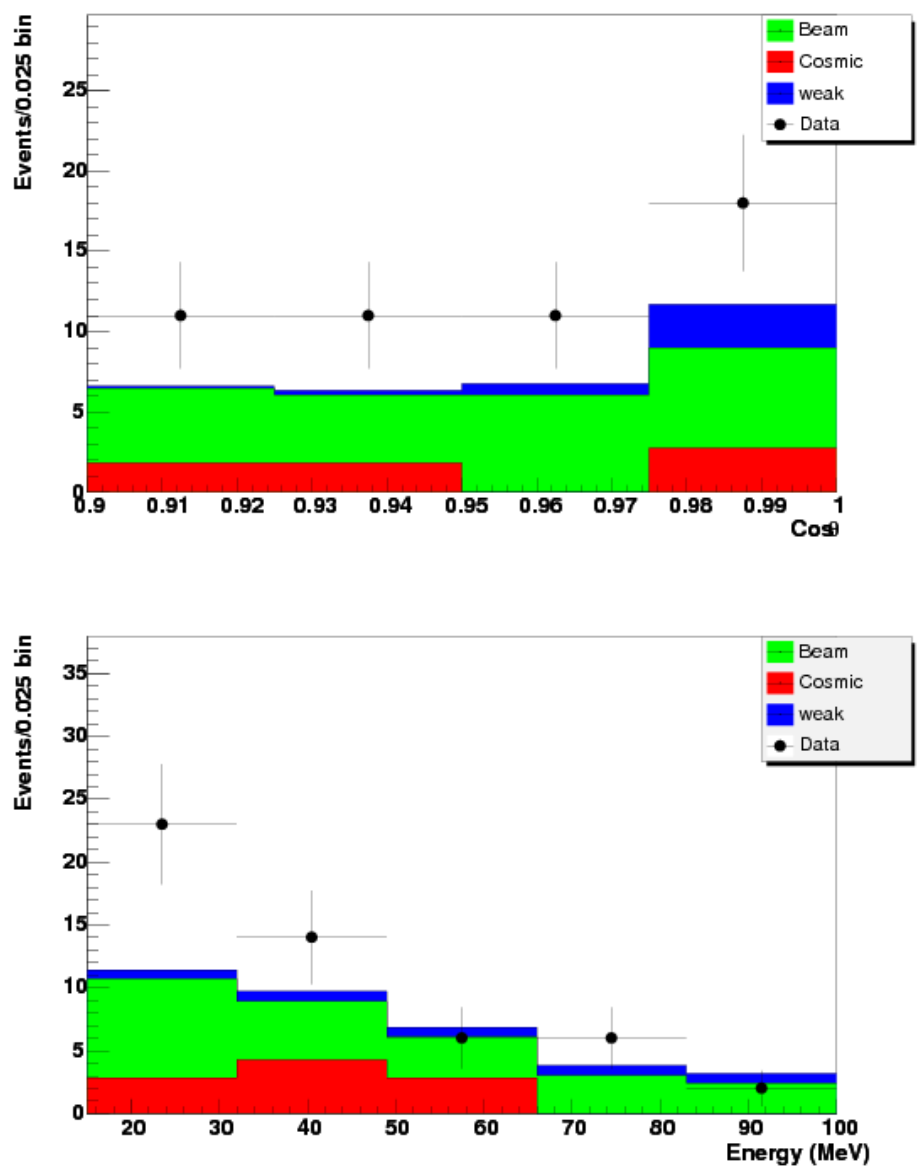

FIGURE 5.9. Shown in these plots are the number of candidate electron events as a function of $\cos \theta$ (top) and recoil energy (bottom). The points represent the data with statistical errors, while the colored histograms are the different sources of electrons normalized to the data protons-on-target. The blue histogram shows the expected signal events. The green and red histograms show respectively the beam related background and the cosmic related background. 

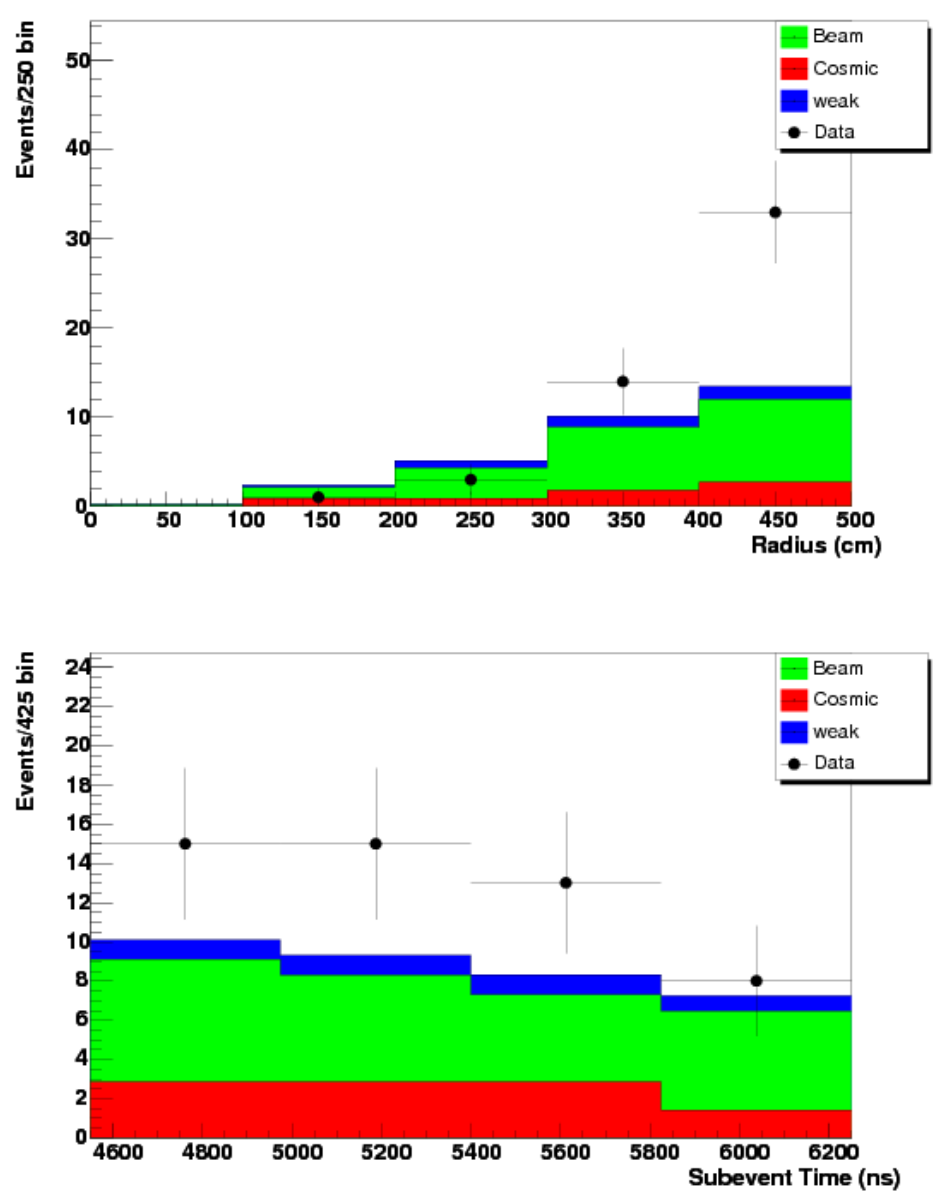

FIGURE 5.10. Data and Monte Carlo distribution of radius (top) and time (bottom) for events passing all the cuts. The points represent the data with statistical errors, while the colored histograms are the different sources of electrons normalized to the data protons-on-target. The blue histogram shows the expected signal events. The green and red histograms show respectively the beam related background and the cosmic related background. 

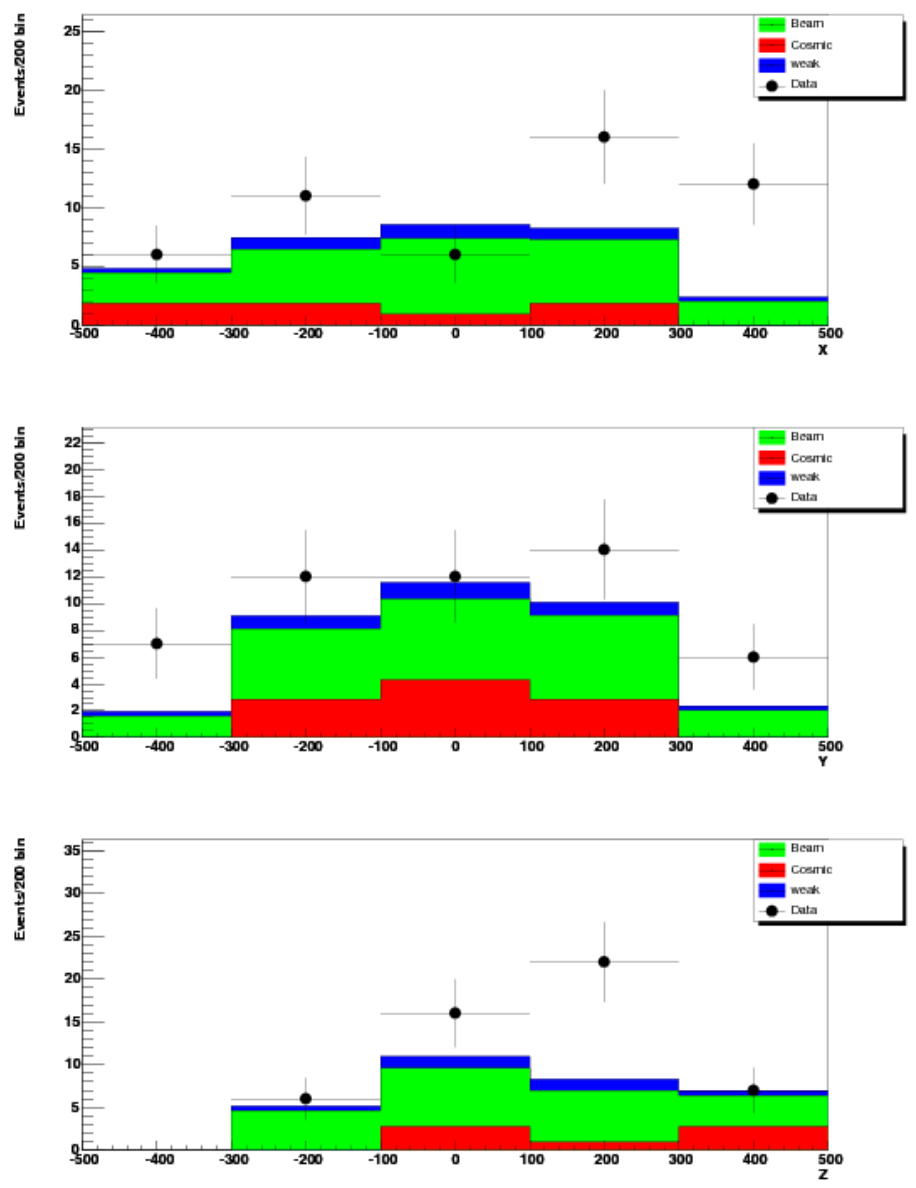

FIGURE 5.11. Data and Monte Carlo distribution of spatial coordinates for events passing all the cuts. The points represent the data with statistical errors, while the colored histograms are the different sources of electrons normalized to the data protons-on-target. The blue histogram shows the expected signal events. The green and red histograms show respectively the beam related background and the cosmic related background. 

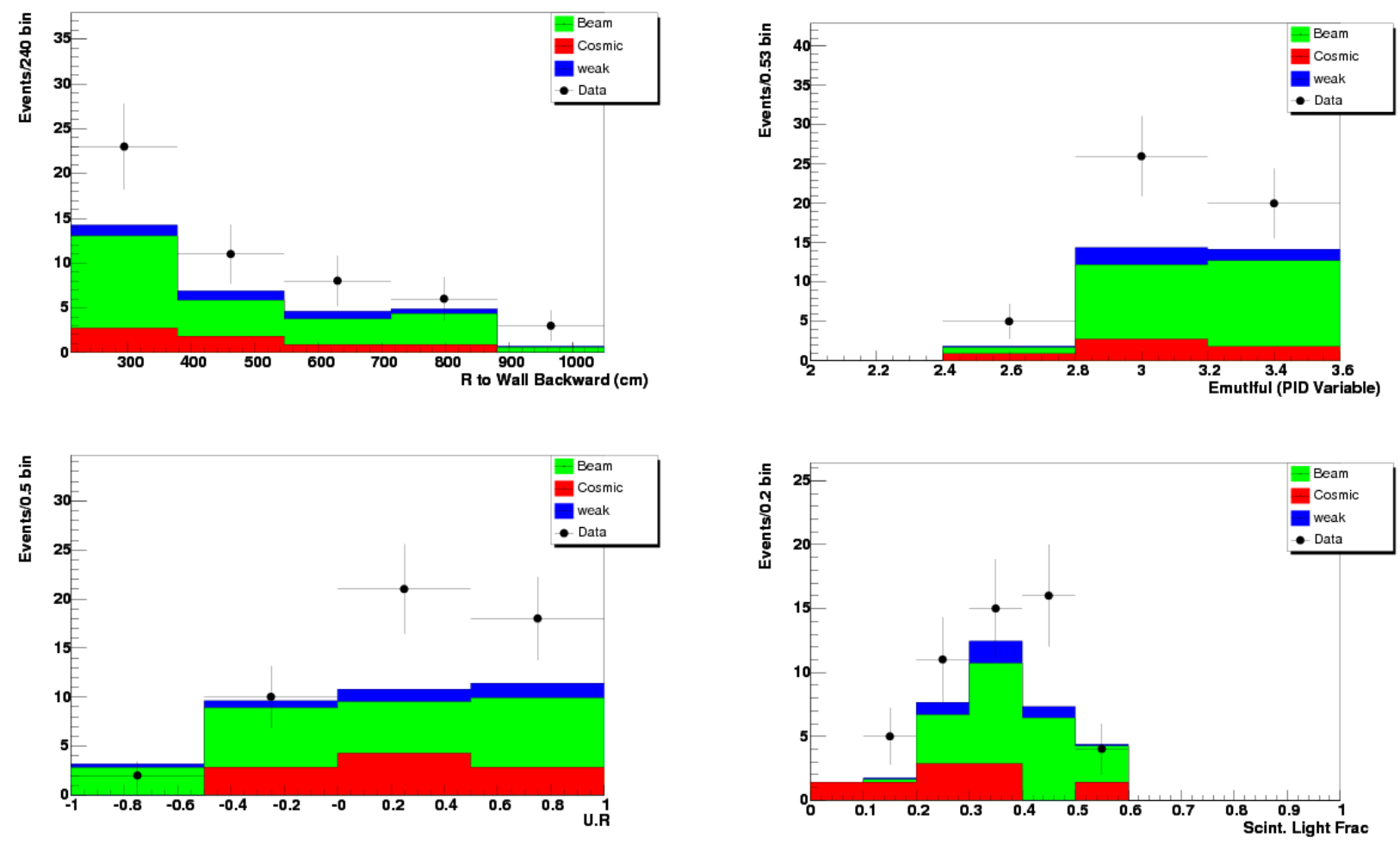

FIGURE 5.12. Data and Monte Carlo distribution of variables used in the event selection. The points represent the data with statistical errors, while the colored histograms are the different sources of electrons normalized to the data protons-on-target. The blue histogram shows the expected signal events. The green and red histograms show respectively the beam related background and the cosmic related background. 
a flat background in $\cos \theta$. Besides $\nu_{\mu} e$ events, no other physical process produces low energy electrons or gammas with a significant $\cos \theta$ variation in the interval $\cos \theta>0.90$. The assumption on the background shape is supported by the bottom panels of figure 5.13. They show the angular distribution of the cosmic related background on the left and the beam related background on the right. Although limited by statistics (to get more statistics the plot of the cosmic background was extended to 0.80 ), the 2 shapes are consistent with being flat over the range of interest. A likelihood function defined in equation (5.10) is fitted to the data to determine $\mathrm{C}$, the fraction of data events that are flat background. Then (1-C) is the fraction that is due to elastic scattering. For a given value of $\mathrm{C}$, the probability distribution normalized in the region $\cos \theta>0.90$ is:

$$
P(C, \cos \theta)=N_{1} C+N_{2}(1-C) g(\cos \theta)
$$

where $N_{1}$ and $N_{2}$ are the normalizations constants. The actual function minimized is:

$$
-\ln \mathcal{L}(C, \cos \theta)=-\sum_{i=1}^{\text {events }} P\left(C, \cos \theta_{i}\right)
$$

The top panel of figure 5.13 shows the angular distribution of Monte Carlo $\nu_{\mu} e$ events fitted to obtain the function $\mathrm{g}(\cos \theta)$. The minimization of $-\ln L$ finds that $70.0 \% \pm 12.3 \%$ of the data events are background events. The error is obtained from finding the values of $\mathrm{C}$ when the negative log likelihood increases by 0.5 . It follows that the data sample of 51 events contains $15.3 \nu_{\mu} e$ events and 35.7 flat background events, which is consistent with the result obtained in section 5.5. The uncertainties on the expected number of events are discussed below. 

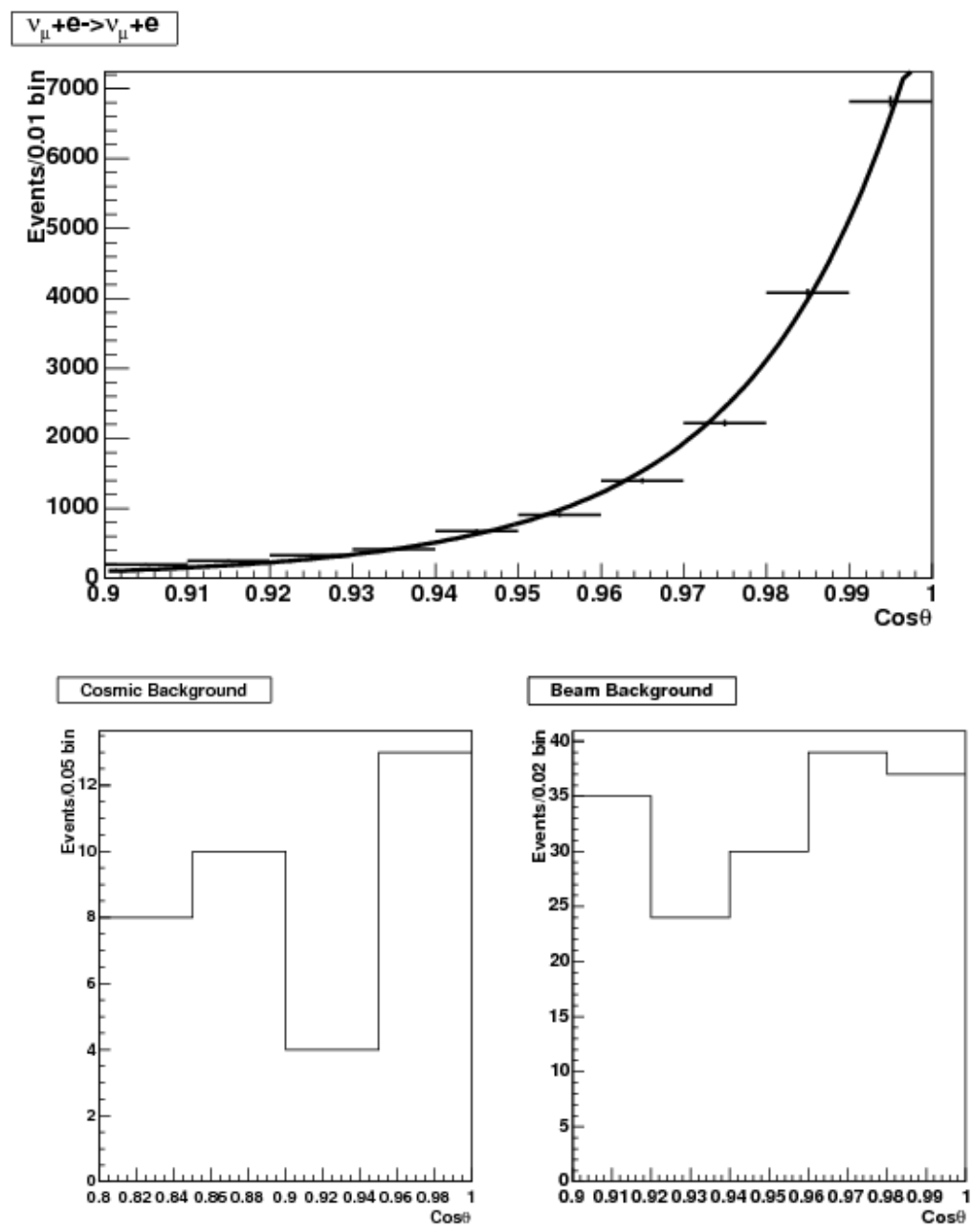

FIGURE 5.13. The top plot shows the $\cos \theta$ distribution of simulated $\nu_{\mu}$ e events fitted with a function that will be used to construct the likelihood function. The two bottom plots are the angular distribution of the cosmic background (left) and beam background (right). Within statistics, those plots are consistent with being flat. 


\subsection{Significance of the Excess}

\subsubsection{Significance For The Counting Method}

In the counting method of extracting the signal, statistical errors on the cosmic ray events and beam events (dirt and In-tank) are a major source of uncertainty. As listed in in table 5.2, the cosmic background sample has seven events before normalization, corresponding to 7.2 expected events in the data sample. Therefore, the uncertainty in the expected number of cosmic background is $\frac{7.2}{\sqrt{7}} \sim 2.7$.

Similarly, there are 105 beam related background events (dirt and In-tank) before normalization. With 21 expected events in the data, the uncertainty in the beam

related background is $\frac{21}{\sqrt{105}} \sim 2.05$. From these two sources it follows that there is a statistical uncertainty in the predicted number of background events in the order of $\sqrt{(2.7)^{2}+(2.05)^{2}} \sim 3.4$ events. The largest source of error is systematic and arises from uncertainty in the normalization of the Monte Carlo events. For the oscillation analysis, the systematic errors associated with the neutrino fluxes, the detector model, and the neutrino cross sections are each considered as independent groups [31]. For each group, an individual matrix that includes the full correlations between the systematic parameters was formed. The oscillation signal and the uncertainties, statistical and systematics, are obtained from a $\chi^{2}$ mininization of the final covariance matrix which is the sum of the individual matrices.

For this thesis, because the cross section of the $\nu_{\mu}$ e interactions is well known [6], we consider only the systematic errors associated with the neutrino fluxes and the detector model. This normalization error is set to $15 \%$ which is lower than the error from the oscillation analysis. However, it is important to note that the $\cos \theta$ fitting procedure discussed below is used to obtain a limit on the magnetic moment and is insensitive to this normalization error. 
Therefore, with 25.6 predicted beam events, there is a systematic uncertainty of 3.8 events from the normalization of the Monte Carlo events, which added in quadrature with the statistical uncertainty gives 5.1 events as the total uncertainty in the predicted number of background events. Finally, to determine the error on the excess, we consider the statistical uncertainty in the observed number of events which is equal to $\sqrt{51}$. Thus, the predicted number of events from this method is $32.8 \pm 5.1$ for an excess of $18.2 \pm 8.8$ events.

\subsubsection{Significance For the $\operatorname{Cos} \theta$ Fitting Procedure - Sources of Statistical Errors}

For the $\cos \theta$ fitting procedure, we considered two main sources of statistical errors . The first source arises from the uncertainty on the measured fraction of events in the data sample. As reported in section $5.7,70.0 \% \pm 12.3 \%$ of the 51 data events are background events, meaning that there are about 15.3 signal events. Thus, the statistical error on the signal fraction is:

$$
1^{\text {st }} \text { stat. error }=51 \times 0.123 \sim 6.3 \text { events. }
$$

The other source of statistical error comes from the uncertainty on the measured number of data events. This uncertainty is obtained from fluctuating the number of signal events by $1 / \sqrt{51}$, and it corresponds to:

$$
2^{\text {nd }} \text { stat. error }=15.3 \times \frac{1}{\sqrt{51}} \sim 2.1 \text { events }
$$

The total statistical error is therefore:

$$
\text { stat. error }=\sqrt{6.3^{2}+2.1^{2}} \sim 6.6 \text { events }
$$




\section{- Sources of Systematic Errors}

The uncertainty in the angular distributions for the background events and the electroweak events constitutes the dominant sources of systematic uncertainty in this procedure.

For the background events, the uncertainty is obtained from assuming that the angular distribution is no longer flat, but varies according to a function $\mathrm{h}(\cos \theta)$ given by:

$$
h(\cos \theta)=a+b(1-\cos \theta)
$$

A fit to the angular distribution of the beam related background events yields:

$$
\begin{aligned}
& a=34.59 \pm 5.56 \\
& b=14.64 \pm 49.27 .
\end{aligned}
$$

In this case, the probability function in equation (5.9) becomes:

$$
P(C, \cos \theta)=N_{1} h(\cos \theta) C+N_{2}(1-C) g(\cos \theta),
$$

from which a likelihood function similar to equation (5.10) can be constructed. A minimization of this likelihood function indicates that $69.4 \%$ of the 51 data events are background events, marginally different from the initial minimization where we obtained $70 \%$.

Since the parameter b as given in equation (5.14) controls the variation of the angular distribution, its fit values are used to obtained the systematic errors. Thus the uncertainty associated with a variation of the angular distribution for the background events is:

$$
1^{\text {st }} \text { syst. error }=\left((70-69.4) / 100 \times \frac{49.27}{14.64}\right) \times 51 \sim 1.03 \text { event. }
$$




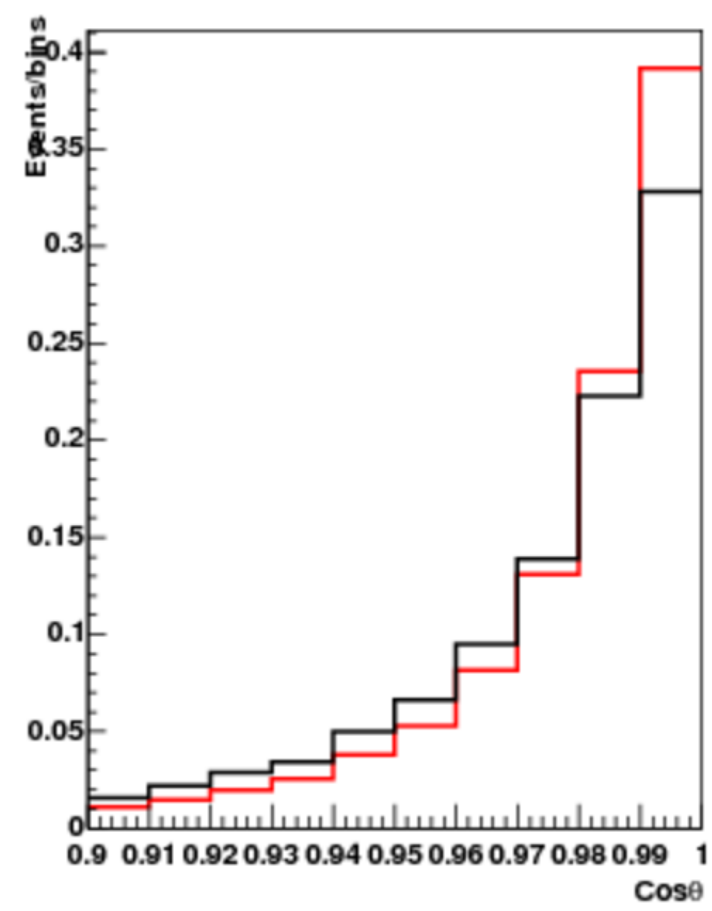

FIGURE 5.14. Angular distribution of the electroweak events in red and electroweak events reweighted by $1 / \mathrm{T}$ in black.

The variation of the angular distribution for the elastic scattering events is obtained from reweighting the distribution by $1 / \mathrm{T}$, where $\mathrm{T}$ is the kinetic energy of the recoil electron from the process $\nu_{\mu}+e \rightarrow \nu_{\mu}+e$. This procedure yields the difference in the angular distribution of the signal events for the extreme cases of only electroweak events and only electromagnetic events. Figure 5.14 shows in red the angular distribution of the electroweak events, and in black the distribution for the same events reweighted by $1 / \mathrm{T}$. The resulting distribution is first fitted to obtain a modified function $\mathrm{g}(\cos \theta)$ which is used to construct a modified likelihood function. The minimization of this likelihood function yields that $63.6 \%$ of the data sample are background events, and thus, the uncertainty error associated with a variation of the angular distribution for the electroweak events is:

$$
2^{\text {nd }} \text { syst. } \text { error }=(70-63.6) / 100 \times 51 \sim 3.3 \text { events. }
$$


Taking into consideration the $15 \%$ normalization error for elastic scattering events, $15.3 \times 0.15 \sim 2.3$ events, the total systematic uncertainty is:

$$
\text { syst. error }=\sqrt{(1.03)^{2}+(3.3)^{2}+(2.3)^{2}} \sim 4.1 \text { events. }
$$

The expected number of signal events in the data sample is therefore $15.3 \pm 6.6$ (stat) \pm 4.1 (syst), significantly larger than the expected 4.6 events due to weak interactions as reported in table 5.6, leading to an excess of 10.7 events.

\subsection{Reconstruction Bias}

As a check, we ruled out a reconstruction bias as the source of the excess. This is

confirmed by figure 5.15 which shows the angular distribution of Michel electrons. As expected, the distribution is flat, with no arbitrary peaking at high $\cos \theta$.

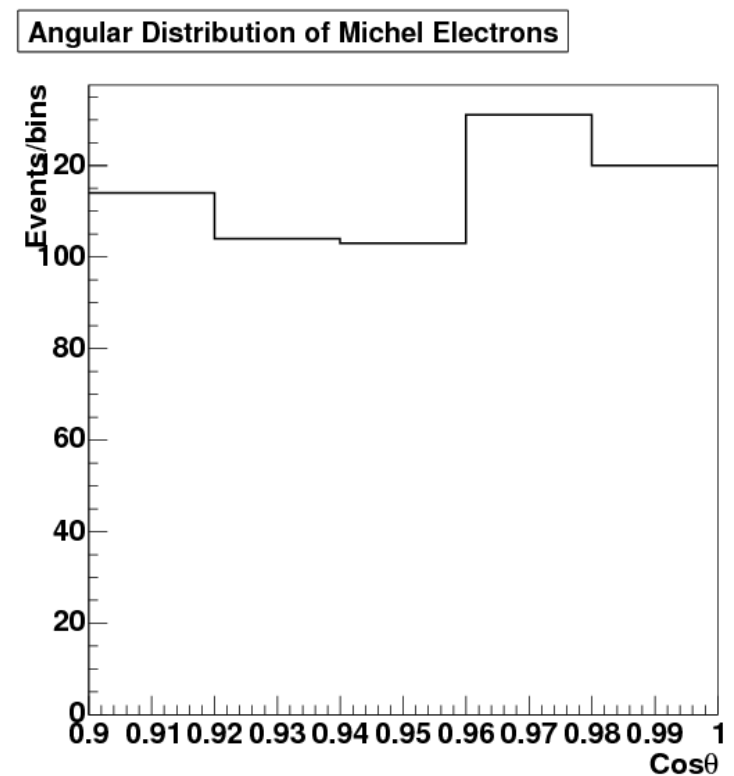

FIGURE 5.15. Angular distribution of Michel electrons. The flatness of the distribution indicates no bias in reconstruction. 


\subsection{Results}

To estimate the neutrino magnetic moment, one can consider the ratio $\mathrm{R}$ of the electromagnetic cross section to the weak cross section:

$$
R=\frac{(d \sigma / d T)^{E M}}{(d \sigma / d T)^{W e a k}}
$$

where $\left(\frac{d \sigma}{d T}\right)^{E M}$ is given by equation (5.1) and $\left(\frac{d \sigma}{d T}\right)^{W e a k}$ by equation (5.2). It can be shown that for $\mathrm{T} \ll \mathrm{E}_{\nu}$ :

$$
R \cong \frac{f^{2} \times 2.5 \times 10^{-25}\left(\frac{1}{T}\right)}{19.04 \times 10^{-46}}
$$

where

$$
f=\frac{\mu_{\nu}}{\mu_{B}}
$$

To determine the value of $\mathrm{f}$ at the $90 \%$ confidence level (CL), we generated 10000 fake experiments for different values of $\mathrm{f}$, each following a Gaussian distribution. The mean of the Gaussian is the total expected number of events from elastic scattering, and the width is 7.8 events, the sum of the statistical uncertainty and the systematic uncertainty added in quadrature. The upper limit of $\mathrm{f}$ at the $90 \% \mathrm{CL}$ is the value that has $90 \%$ of the experiments with more events than the observed signal of 15.3 events. As shown in figure 5.16, this value of $\mathrm{f}$ is $12.7 \times 10^{-10}$, which according to equation $(5.23)$ can be interpreted as: $\mu_{\nu}<12.7 \times 10^{-10} \mu_{B}$ at the $90 \%$ CL.

A limit on the neutrino magnetic moment has been obtained from the $\nu_{\mu} e \rightarrow \nu_{\mu} e$ interaction. From a total of 51 data events, we observed $15.3 \nu_{\mu}$ e events leading to a limit of $12.7 \times 10^{-10} \mu_{B}$ at the $90 \%$ confidence level. This limit is higher than the current best limit of $6.8 \times 10^{-10} \mu_{\nu}$ [18]. We note that our limit would have 


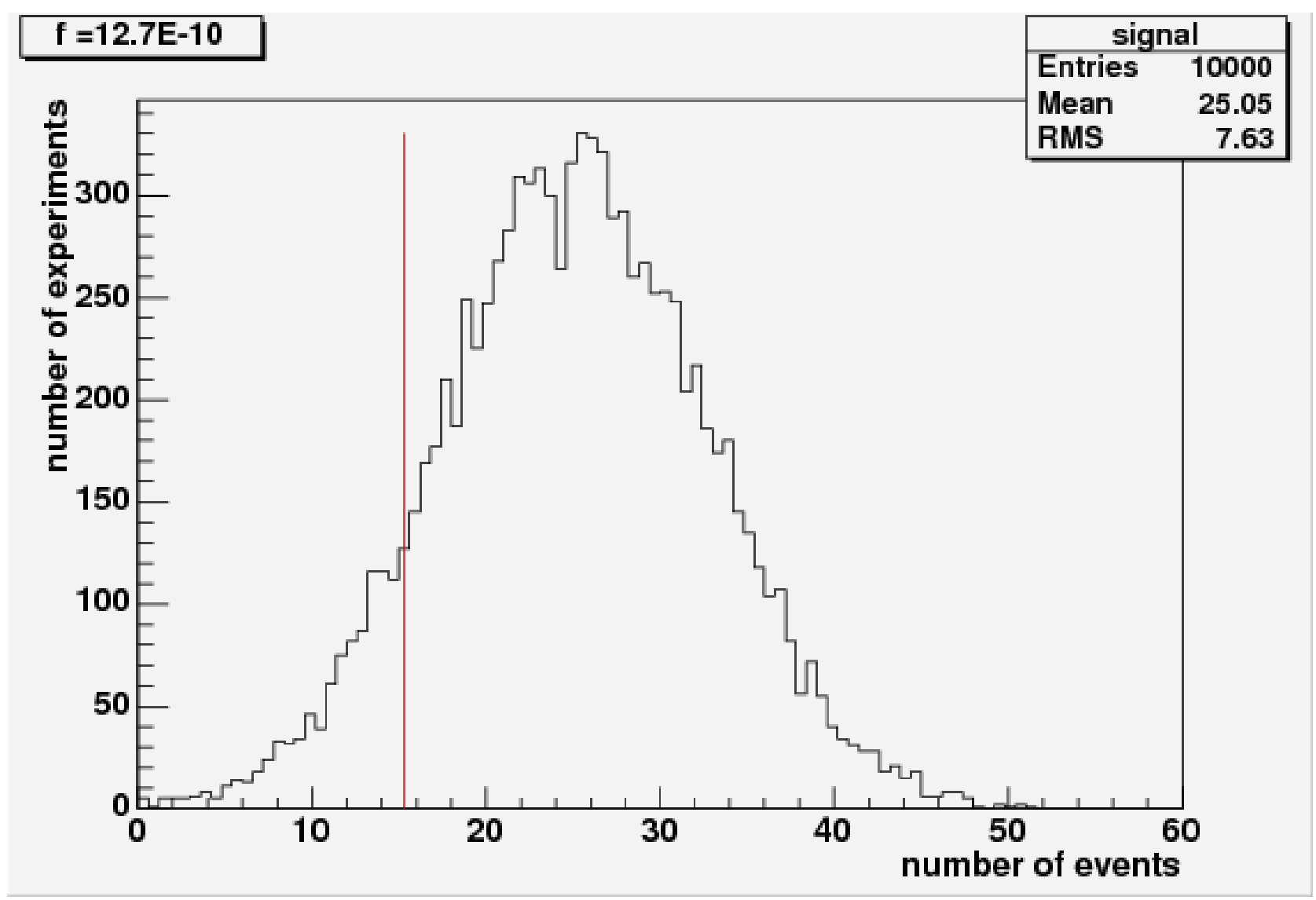

FIGURE 5.16. Gaussian distribution for 10000 fake experiments with $\mathrm{f}=12.7 \times 10^{-10}$. The red line is at 15.3 showing that $90 \%$ of the time, we would see an excess of at least 15.3 events if the magnetic moment were $12.7 \times 10^{-10} \mu_{\nu}$. 
been comparable if we had not observed an unexpected excess of forward going electrons.

MiniBooNE in its oscillation results reported an excess of low energy events (96 $\pm 17 \pm 20$ events) for neutrino energy between $300 \mathrm{MeV}$ and $475 \mathrm{MeV}$. The source of this excess is still under investigation and might well be related to the excess of forward electrons events that we observed between $15 \mathrm{MeV}$ and $100 \mathrm{MeV}$. Since an unexplained excess of events is observed for these two analyses, in chapter 6 , we will perform a search for elastic scattering electrons above $100 \mathrm{MeV}$. 


\section{Chapter 6}

\section{High Energy Elastic Scattering Events}

For this study, a new sample of 9 million beam MonteCarlo events was generated using the NUANCE v3 generator. This MonteCarlo sample is an updated version compared to the one used in chapter 5 and includes $301 \nu_{\mu}$ e events which we will refer to as the signal events. The correponding proton-on-target (POT) of the simulated events is 4.1E21, whereas all the available data events were used, corresponding to 5.68E20 POT. The data POT for this analysis is larger than for the low energy analysis because the missing information in the data collected prior to fall 2003 was not needed. As explained in chapter 5, at low energy the background is dominated by Michel electrons from the decay of cosmic ray muons, hence the necessity of the past time variable to separate low energy $\nu_{\mu}$ e events from the Michel electron background. Here, the Michel background is easily removed by requiring the number of hit PMTs in the main tank be above 200 .

\subsection{High Energy $\nu_{\mu}$ e Event Selection}

The high energy $\nu_{\mu}$ e event selection begins with a basic set of cuts similar to the low energy $\nu_{\mu}$ e event selection:

- Only one subevent

- $\mathrm{N}_{\text {veto }}<6$

- $\mathrm{R}<500 \mathrm{~cm}$

The motivation for this cut is identical to the motivations provided in chapter 5 . 


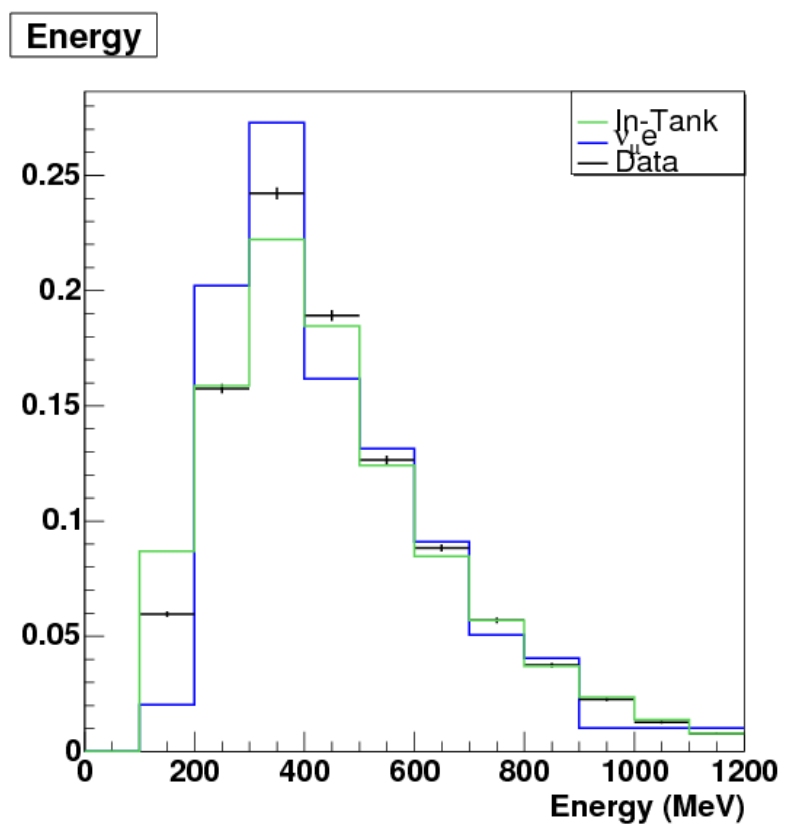

FIGURE 6.1. Energy distribution for events passing the precuts, except the energy cut. The data events are shown in black dots, the $\nu_{\mu}$ e events in blue color and the in-tank in green color.

- $\mathrm{N}_{\text {tank }}>200$

This cut is to remove the Michel electrons mostly from decays of cosmic ray muons.

- Electron Energy $<800 \mathrm{MeV}$.

This cut is required because above this energy, the background rate dominates the $\nu_{\mu}$ e events.

After these precuts, the Monte Carlo simulation shows that the dominant background sources are $\mathrm{NC} \pi^{0}$ events and $\nu_{\mu} \mathrm{CCQE}$ events where the decay electron from the final muon goes undetected. Sections 6.3 and 6.4 describe two independent methods, each using a different set of PID variables to decrease the two main sources of background, to obtain a sample that will be dominated by the signal events. It is also important to note that because of the $\mathrm{T}$ dependence of the angular 
distribution as expressed in equation (5.3), the elastic scattering signal is expected to occur mostly in the region $\cos \theta>0.99$ for these higher energy electrons.

\subsection{Dirt Event Rate In the Data Sample}

As mentioned in chapter 5, dirt events are interactions of neutrinos with the material surrounding the detector which produce other particles, including photons that can enter the detector and be misidentified as electrons.

The initial simulation of beam Monte Carlo events used for this analysis does not contain dirt events. For the analysis based on the PIDs variables from Boosting Decision Tree (BDT), we estimate the rate of dirt events by using the Monte Carlo simulation discussed in chapter 5 . The variable rtowall, also defined in chapter 5 , is used to separate dirt events from other beam related events. The top left plot of figure 6.2 shows the rtowall distribution for events that passed the precuts. The samples presented in the plot are dirt Monte Carlo (red), elastic scattering (blue) and data (black) events. The top right plot shows the rtowall distribution where a rtowall $>300 \mathrm{~cm}$ cut has been applied.

The number of dirt events passing the precuts is 8964, which is reduced to 547 after the rtowall cut. The rate of dirt events in the data sample is obtained after properly normalizing the number of events that pass the rtowall cuts and the PID cuts to the data POT.

For the analysis with the PIDs from the Track-Based Algorithm, the rate of dirt events is obtained from the oscillation analysis where the exact same cuts were applied.

\subsection{Analysis Based On PID Variables From The Boosting Decision Tree (BDT) Algorithm}

For the analysis that relies on the boosting PIDs, cuts were imposed on the PID variables ELMU and ELPI, both created from the procedure outlined in chap- 

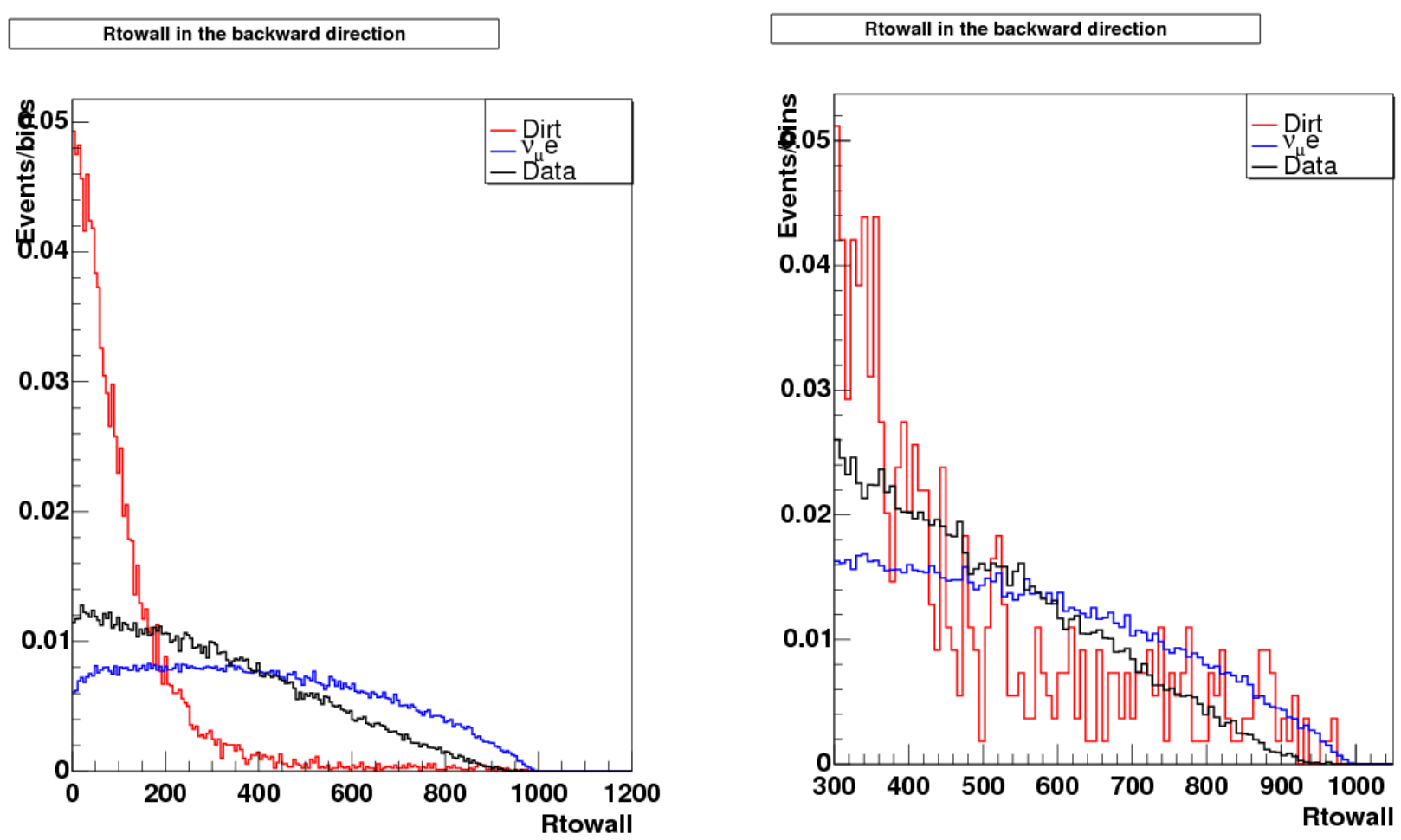

FIGURE 6.2. Left: Rtowall distribution for data events (black), elastic scattering events (blue) and dirt events (red) passing the precuts. Right: Same distribution after a rtowall cut was applied.The distributions are normalized to unit area.

ter 4. The variable ELMU was developed to distinguish electron-like events from muon-like events, while ELPI was developed to distinguish electron-like events from events having the characteristics of $\mathrm{NC} \pi^{0}$. The two cuts are:

- $\mathrm{ELMU}>0.0$

- ELPI $>-0.8$

After these cuts, we also require that the reconstructed mass of any $\pi^{0}$ be less than $80 \mathrm{MeV} / \mathrm{c}^{2}$ to further decrease the background contamination due to $\mathrm{NC} \pi^{0}$ events.

The left plot in figure 6.3 shows the ELMU distribution for events that passed the precuts and the rtowall cut. It also shows on the right the ELPI distribution for events that satisfied the precuts, the rtowall and the ELMU PID cuts. The reconstructed $\pi^{0}$ mass distribution for events that passed these cuts and the ELPI 

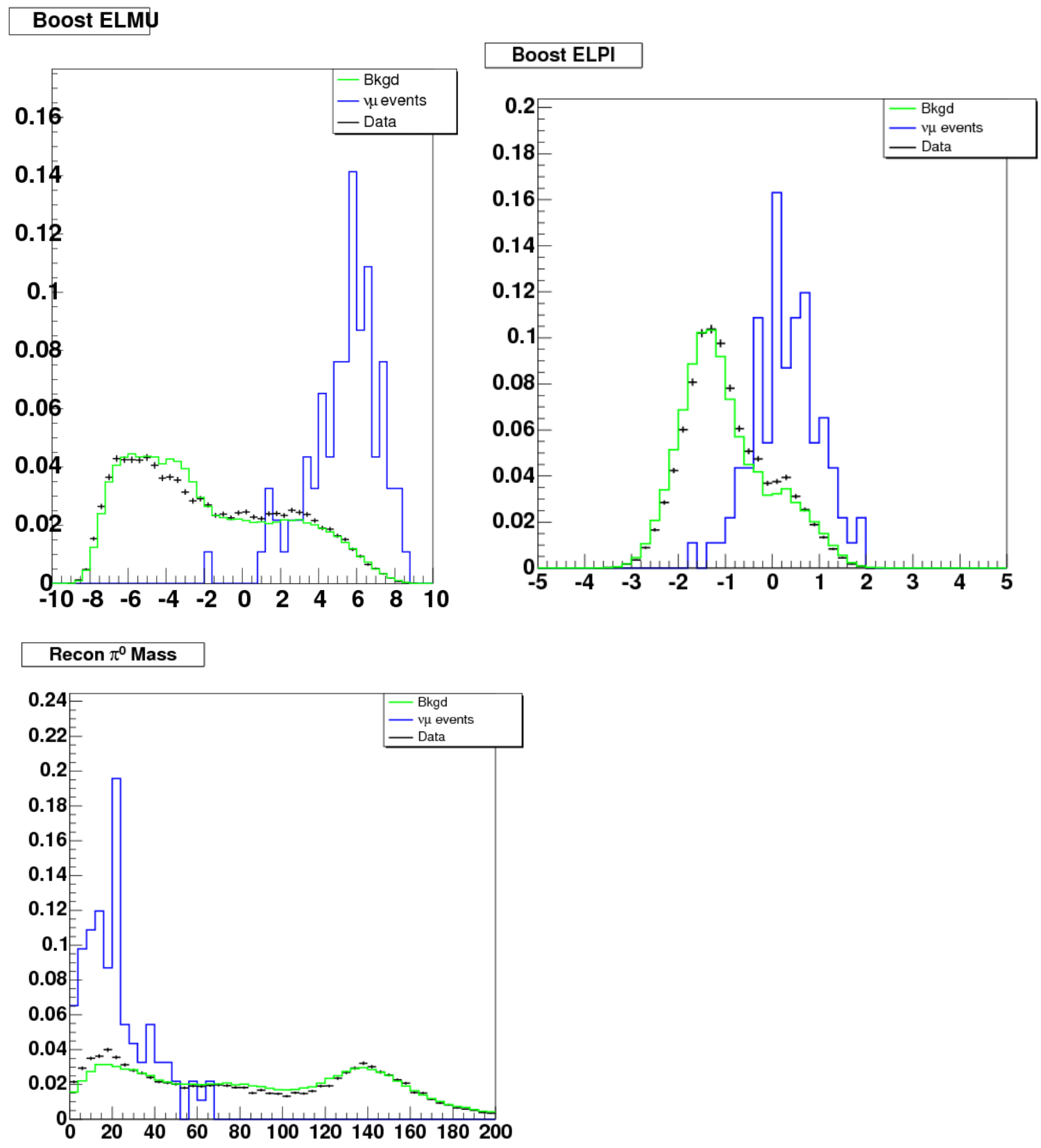

FIGURE 6.3. The ELMU PID distribution for events that passed the precuts and the rtowall cut is shown on the left. On the right is the ELPI distribution for events passing the precuts, rtowall and the ELMU cut. The $\pi^{0}$ mass distribution of events passing the precuts, rtowall and both PID cuts is shown in the bottom. In all of these plots the blue (green) curve shows the signal (background) events. The data are in black with statistical error. 
cuts is shown in the bottom of the same figure. For all these plots, the blue curve represent the signal events, the green curve is the background events and the data events are represented by black points with statistical errors. Table 6.3 lists the survival fraction of the events after the PID cuts and the mass cut are applied sequentially.

TABLE 6.1. Survival fraction of background events, signal events and data after the PID cuts and the $\pi^{0}$ mass cut were applied sequentially.

\begin{tabular}{|c|c|c|c|}
\hline & In-tank & Signal & Data. \\
\hline Precuts+rtowall & $100 \%$ & $100 \%$ & $100 \%$ \\
\hline ELMU $>0$ & $31.8 \%$ & $98.9 \%$ & $34.3 \%$ \\
\hline ELPI $>-0.8$ & $6.8 \%$ & $93.4 \%$ & $7.4 \%$ \\
\hline$\pi^{0}$ mass $>80$ & $2.8 \%$ & $93.4 \%$ & $3.6 \%$ \\
\hline
\end{tabular}

\section{- Result of the Analysis}

The search for an excess is performed for different intervals of $\cos \theta$, after normalizing the Monte Carlo signal and background events that have satisfied all the cuts to the data POT. Table 6.3 summarizes the expected number of In-tank, dirt and signal events that have passed the precuts, rtowall and the boosting PID cuts for 5.68E20 POT.

TABLE 6.2. The table summarizes the number of signal, in-tank and dirt events for 5.68E20 POT. The cuts applied are the precuts, rtowall, the cuts on Boosting PID variables and the $\pi^{0}$ mass.

\begin{tabular}{|c||c|c|c|}
\hline & $\cos \theta>0.90$ & $\cos \theta>0.98$ & $\cos \theta>0.99$ \\
\hline In-tank & 144.8 & 31.3 & 17.4 \\
\hline dirt & 13.5 & 2.9 & 1.6 \\
\hline$\nu_{\mu} \mathrm{e}$ & 11.9 & 9.7 & 8.9 \\
\hline
\end{tabular}

Figure 6.4 shows the angular distribution (left), the energy distribution (right), and the radius distribution (bottom) for data events (black), elastic scattering events (blue), and in-tank (green). The systematic error for this analysis arises 

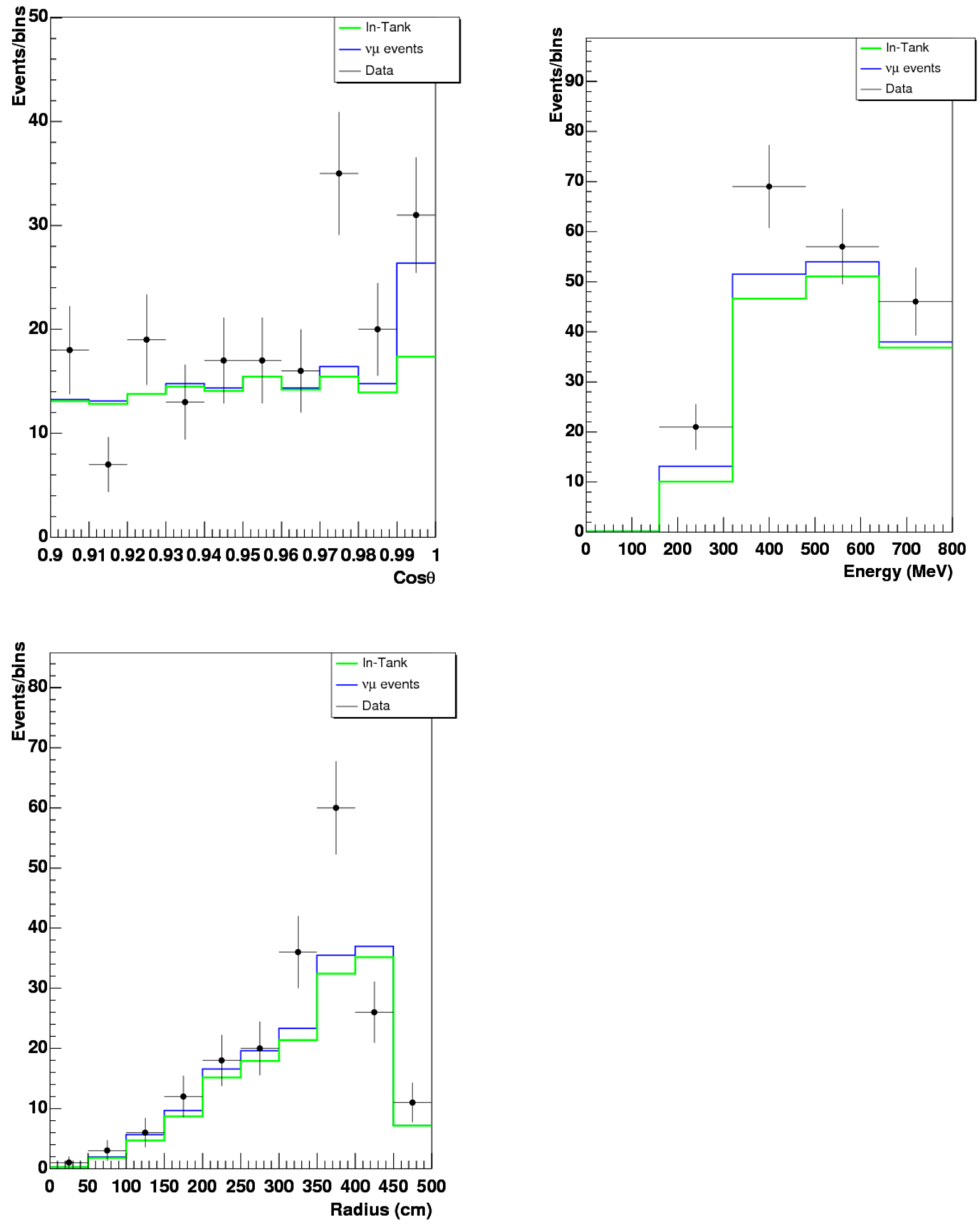

FIGURE 6.4. Angular distribution, energy distribution and radius distribution events passing the precuts, the rtowall cuts and the BDT pid cuts. The data events are shown in black dots, the $\nu_{\mu}$ e events in blue color and the in-tank in green color. 
from the absolute normalization of the Monte Carlo sample which is in turn related to the uncertainty to the neutrino flux prediction and the accuracy of our events simulations. As in chapter 5 , we assigned a $15 \%$ systematic uncertainty to the normalization. Table 6.3 gives the expected number of beam events with both statistical and systematic errors and the measured number of data events passing the precuts, rtowall and the boosting PID cuts. The table also shows the measured excess and the significance of the deviation as obtained from the following equation:

$$
N_{\sigma}=\frac{N_{\text {data }}-N_{\text {expected }}}{\sqrt{\sigma_{\text {stat }}^{2}+\sigma_{\text {syst }}^{2}}}
$$

TABLE 6.3. The table summarizes the number of signal and background events for 5.68E20 POT. The cuts applied are the precuts, rtowall and the cuts on Boosting PID variables.

\begin{tabular}{|c||c|c|c|}
\hline & $\cos \theta>0.90$ & $\cos \theta>0.98$ & $\cos \theta>0.99$ \\
\hline Data & 193 & 51 & 31 \\
\hline Beam Evts & $170.1 \pm 13.9($ stat $) \pm 25.5($ syst $)$ & $44.0 \pm 7.1$ (stat) $\pm 6.6($ syst $)$ & $27.9 \pm 5.5$ (stat) \pm 4.2 (syst) \\
\hline \hline Excess & $22.8 \pm 29.0$ & $6.9 \pm 9.7$ & $3.0 \pm 6.9$ \\
\hline Significance & $0.8 \sigma$ & $0.7 \sigma$ & $0.4 \sigma$ \\
\hline
\end{tabular}

\subsection{Analysis Based On PID Variables From the Track-Based Algorithm (TBA)}

The equivalent of the variables ELMU and ELPI for the TBA analysis (discussed in section 6.3)are likeemu and likeepi respectively. Figure 6.5 shows the likeemu and likeepi distributions for events that satisfied the precuts. We used the same cuts as for the MiniBooNE oscillation analysis [31] to separate signal events (blue) from background events (green).

As shown in figure 4.2, these likelihood distributions are energy dependent. Therefore the following energy dependent cuts are imposed where the energy E is in GeV. These cuts are also shown in figure 4.2.

- likeemu $>1.355 \times 10^{-2}+\left(3.467 \times 10^{-2}\right) \mathrm{E}+\left(-8.259 \times 10^{-3}\right) \mathrm{E}^{2}$ 
$\log \left(\mathbf{L} \_\right.$e/L_ $\left.\mu\right)$

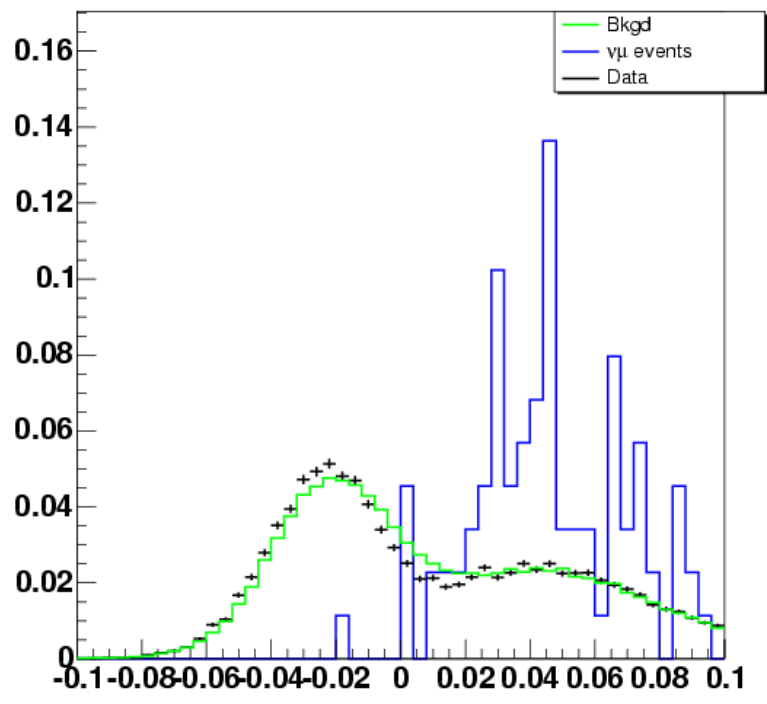

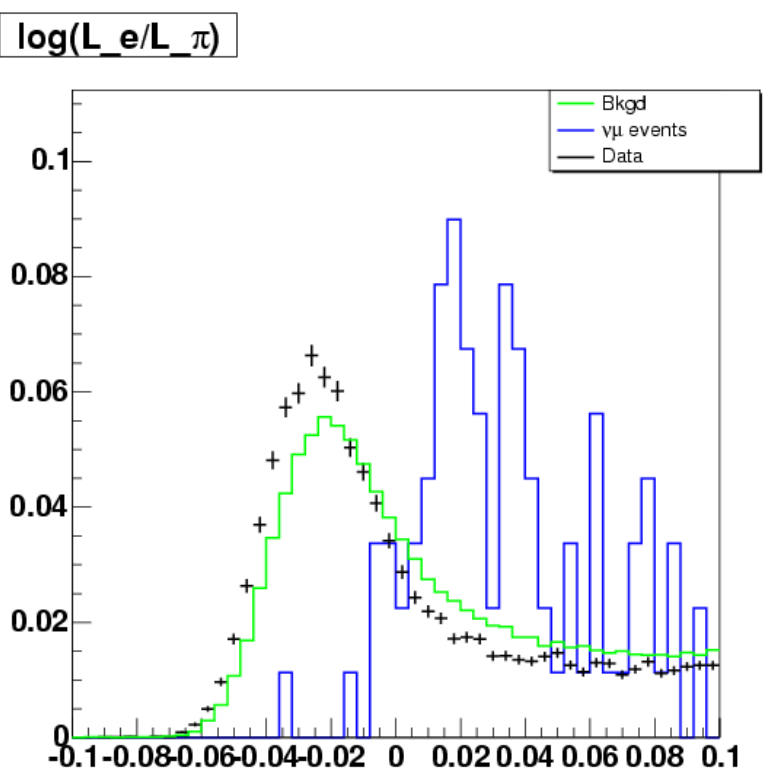

FIGURE 6.5. The likeemu (left) and the likeepi (right) distributions for events passing the precuts are shown. The blue (green) curve shows the signal (background) events. The data are in black with statistical error.

- likeepi $>2.471 \times 10^{-3}+\left(4.115 \times 10^{-3}\right) \mathrm{E}+\left(-2.738 \times 10^{-2}\right) \mathrm{E}^{2}$

- $\pi^{0}$ mass $<3.203 \times 10^{-2}+\left(7.417 \times 10^{-3}\right) \mathrm{E}+(2.738 \mathrm{E}-2) \mathrm{E}^{2}$

Table 6.4 shows the survival fraction for the different event samples when the cuts are applied sequentially.

TABLE 6.4. Survival fraction of background events, signal events and data after the PID cuts and the $\pi^{0}$ mass cut were applied sequentially.

\begin{tabular}{|c|c|c|c|}
\hline & In-tank & Signal & Data. \\
\hline Precuts+rtowall & $100 \%$ & $100 \%$ & $100 \%$ \\
\hline likeemu cut & $37.2 \%$ & $82.6 \%$ & $37.8 \%$ \\
\hline likeepi cut & $37.1 \%$ & $79.3 \%$ & $37.7 \%$ \\
\hline$\pi^{0}$ mass cut & $2.8 \%$ & $68.4 \%$ & $3.4 \%$ \\
\hline
\end{tabular}

\section{- Result of the Analysis}

The results of the analysis based on the TBA cuts are summarized in table 6.4 for different bins of $\cos \theta$. 

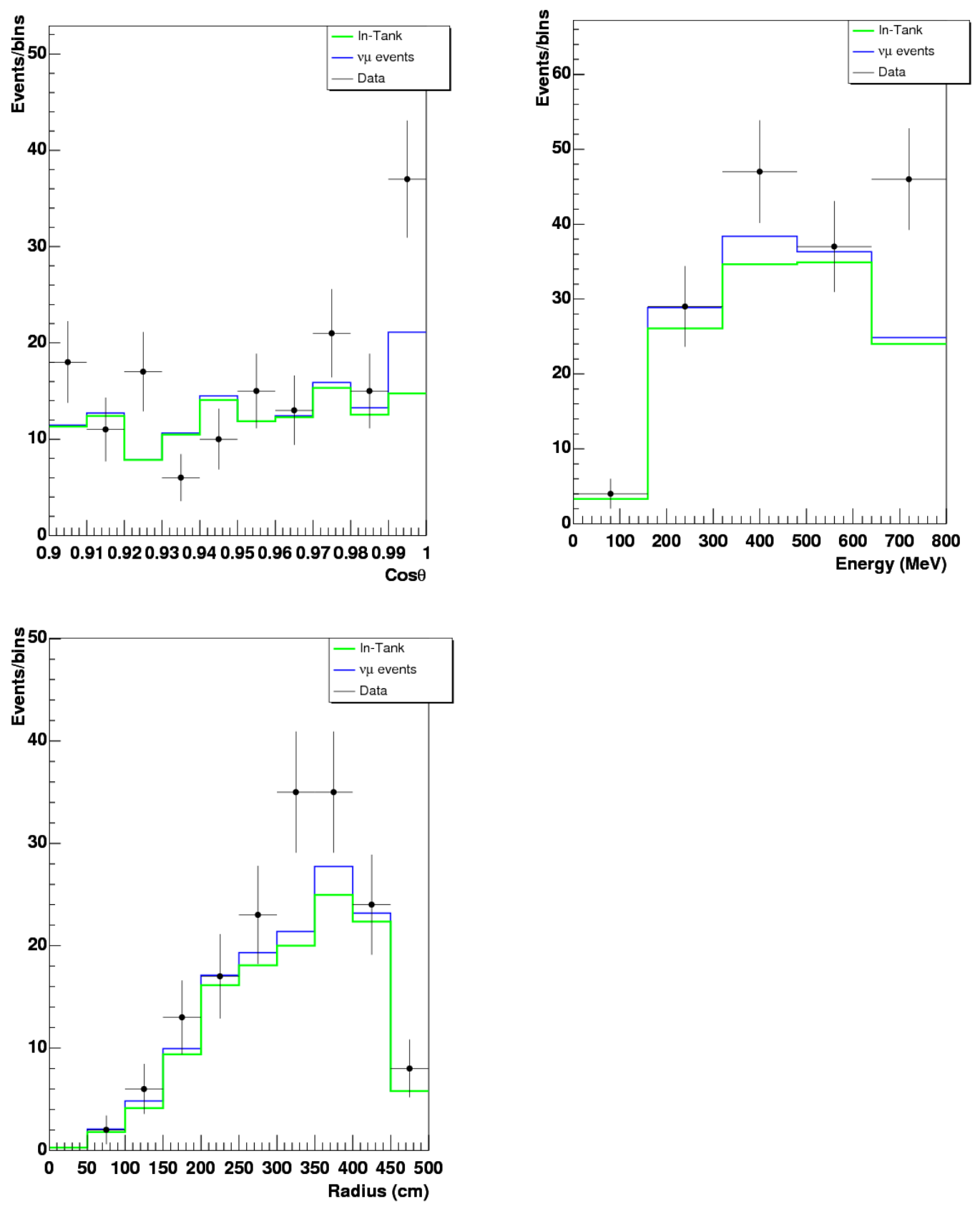

FIGURE 6.6. Angular distribution, energy distribution and radius distribution for events passing the precuts, the rtowall cuts and the TBA pid cuts. The data events are shown in black dots, the $\nu_{\mu}$ e events in blue color and the in-tank in green color 
TABLE 6.5. The table summarizes the number of data, signal and background events for 5.68E20 POT. The cuts applied are the precuts, rtowall and the cuts on TBA PID variables.

\begin{tabular}{|c||c|c|c|}
\hline & $\cos \theta>0.90$ & $\cos \theta>0.98$ & $\cos \theta>0.99$ \\
\hline In-tank & 123 & 27.3 & 14.8 \\
\hline dirt & 9.8 & 3.1 & 2.2 \\
\hline$\nu_{\mu} \mathrm{e}$ & 8.7 & 7 & 6.3 \\
\hline
\end{tabular}

TABLE 6.6. The table summarizes the rate of data, signal and background events for 5.68E20 POT. The cuts applied are the precuts, rtowall and the cuts on the TBA PID variables.

\begin{tabular}{|c||c|c|c|}
\hline & $\cos \theta>0.90$ & $\cos \theta>0.98$ & $\cos \theta>0.99$ \\
\hline Data & 163 & 52 & 37 \\
\hline Beam Evts & $141.4 \pm 12.8($ stat $) \pm 21.2$ (syst) & $37.4 \pm 7.2($ stat $) \pm 5.6($ syst $)$ & $23.3 \pm 6.1($ stat $) \pm 3.5$ (syst) \\
\hline \hline Excess & $21.5 \pm 24.7$ & $14.5 \pm 9.1$ & $13.7 \pm 7.0$ \\
\hline Significance & $0.9 \sigma$ & $1.6 \sigma$ & $1.9 \sigma$ \\
\hline
\end{tabular}

In contrast to the previous analysis, table 6.6 shows that the Monte Carlo prediction is lower than the measured number of events, leading to a larger excess in the most forward region. Figure 6.6 shows the angular distribution, the energy distribution and the radius distribution for data events (black), elastic scattering events (blue) and in-tank events (green).

\subsection{Combined Analysis}

Since we observe a larger excess of forward peaking events in the TBA method than the BDT method, a study of the events that pass both the Boosting cuts and the TBA cuts is required. Table 6.5 lists the rate of the beam events passing the precuts, the rtowall cut and the PID cuts from the two methods. From table 6.8 where we compare the expected number of beam events to the measured number of data events after all the cuts are applied, the excess in the most forward direction is not as signifcant as reported in table 6.6 , but is still larger than the one obtained from the analysis with the BDT. Figure 6.7 shows the angular distribution, energy distribution and radius distribution for the events listed in table in 6.8 . 

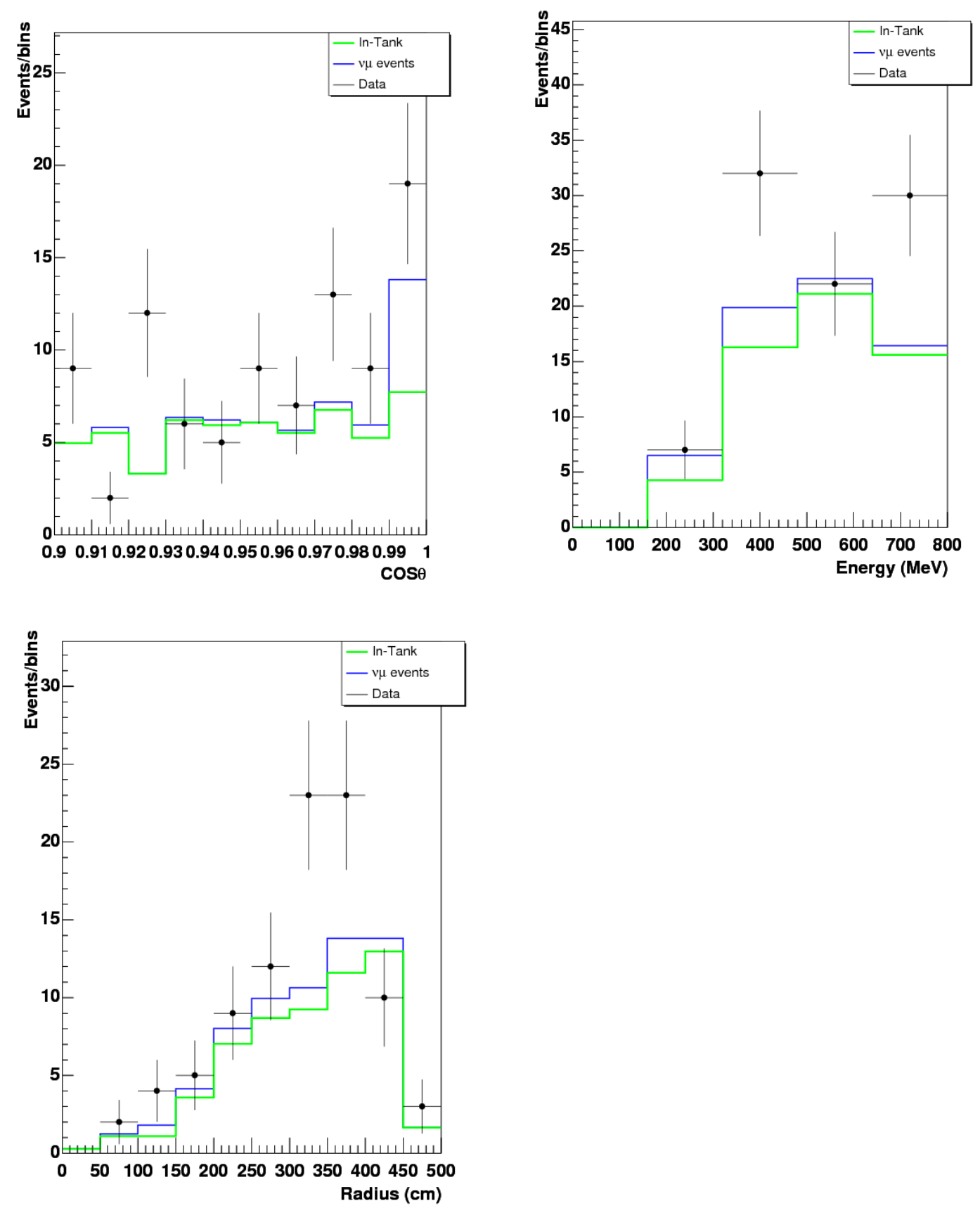

FIGURE 6.7. Angular distribution for events passing the precuts, rtowall and the BDT PID cuts. 
TABLE 6.7. A table summarizing the number of In-tank, dirt and elastic scattering events at different intervl of $\cos \theta$

\begin{tabular}{|c||c|c|c|}
\hline & $\cos \theta>0.90$ & $\cos \theta>0.98$ & $\cos \theta>0.99$ \\
\hline In-tank & 57.3 & 13.0 & 7.7 \\
\hline dirt & 4.9 & 1.1 & 0.7 \\
\hline$\nu_{\mu} \mathrm{e}$ & 8.0 & 6.8 & 6.1 \\
\hline
\end{tabular}

TABLE 6.8. The table summarizes the number of data, signal and background events for 5.68E20 POT. The cuts applied are the precuts, rtowall and the cuts on the TBA PID variables.

\begin{tabular}{|c||c|c|c|}
\hline & $\cos \theta>0.90$ & $\cos \theta>0.98$ & $\cos \theta>0.99$ \\
\hline Data & 91 & 28 & 19 \\
\hline Beam Evts & $70.2 \pm 9.5$ (stat) \pm 10.5 (syst) & $20.8 \pm 5.3($ stat $) \pm 3.1$ (syst) & $14.5 \pm 4.3($ stat $) \pm 2.2$ (syst) \\
\hline \hline Excess & $20.8 \pm 14.2$ & $7.2 \pm 6.1$ & $4.5 \pm 4.8$ \\
\hline Significance & $1.5 \sigma$ & $1.2 \sigma$ & $0.9 \sigma$ \\
\hline
\end{tabular}

\subsection{Summary}

The analysis of the high energy $\nu_{\mu}$ e events does not improve the limit on the neutrino magnetic moment since the electromagnetic cross section decreases as $1 / \mathrm{T}$. But it can give more information on the excess of forward peaking events observed in the low energy regime. From an analysis based on the boosting algorithm PID variables, we observe that the Monte Carlo prediction is consistent with the data events, but on the other hand, we observe an excess of events in the most forward region with a significance of $1.9 \sigma$ when using the PID variables from the TBA algorithm. We also note that an independent analysis by the Los Alamos group using the TBA algorithm observed a similar excess of forward electrons [66].

Given the difference in results from the two independent methods, a selection of events that satisfied the boosting PID cuts and the reconstruction PID cuts was performed. An analysis of these events shows a reduced excess in the forward region but not as significant as the one observed in the low energy region.

The sources of this excess are still under investigation. Statistical fluctuation or error in the normalization of elastic scattering events could be possible explana- 
tions. The forward excess can be also be due to physics processes not simulated in the Monte Carlo. These processes could be an anomalous contribution to the scattering of $\nu_{\mu}$ on electrons, a new particle scattering off an electron, or the electromagentic decay of a light particle via bremsstrahlung in the forward direction. The excess may also be related to the excess of low energy electrons observed by MiniBooNE in the oscillation analysis. MiniBooNE hopes to say more about this excess once we have analyzed the antineutrino data that are now being collected. 


\section{Chapter 7}

\section{Measurement of $\mathrm{CC} \pi^{+}$Cross Section}

In this chapter, we present a measurement of the $\mathrm{CC} \pi^{+}$cross section relative to the CCQE cross section as a function of neutrino energy, ie the ratio of $\mathrm{CC} \pi^{+}$to CCQE events.

For this chapter, $\mathrm{CC} \pi^{+}$refers to both resonant pion production:

$$
\begin{gathered}
\nu_{\mu}+p \rightarrow \mu^{-}+p+\pi^{+} \\
\nu_{\mu}+n \rightarrow \mu^{-}+n+\pi^{+}
\end{gathered}
$$

and coherent interactions:

$$
\nu_{\mu}+A \rightarrow \mu^{-}+A+\pi^{+} .
$$

In general, the $\mathrm{CC} \pi^{+}$cross section is given by:

$$
\sigma_{C C \pi^{+}}=\frac{N_{C C \pi^{+}}}{\phi_{\nu} N_{\text {targets }}}
$$

where $\mathrm{N}_{C C \pi^{+}}$is the background and efficiency corrected number of $\mathrm{CC} \pi^{+}$events measured in the data, $\phi_{\nu}$ is the flux of the incident neutrinos and $\mathrm{N}_{\text {targets }}$ is the number of target nuclei available in the detector. A similar expression can be written for CCQE events. Thus the ratio of the cross section for $\mathrm{CC} \pi^{+}$to the cross section for CCQE at a given neutrino energy is:

$$
\sigma_{C C \pi^{+}}=\frac{N_{C C \pi^{+}}}{N_{C C Q E}} \times \sigma_{C C Q E}
$$

where $\phi_{\nu}$ and $\mathrm{N}_{\text {targets }}$ cancel since they are the same for all the interactions in the detector. Given equation (7.5), the measurement of the cross section ratio reduces to determining the corrected number of $\mathrm{CC} \pi^{+}$and $\mathrm{CCQE}$ events in the data. 


\subsection{Procedure}

Like most analyses, the selection of signal events, in our case $\mathrm{CC} \pi^{+}$, requires setting cuts on specific variables in order to separate signal events from background events. For a given process $\alpha,\left(\mathrm{CCQE}\right.$ or $\left.\mathrm{CC} \pi^{+}\right)$, and a given set of cuts, the signal fraction is determined from the fraction of the number of MonteCarlo events of type $\alpha$ in the $\mathrm{j}^{\text {th }}$ reconstructed energy bin that passed the cuts:

$$
N_{\alpha, \text { cut }}^{M C}\left(E^{R}\right)_{j}=N_{c u t}^{M C}\left(E^{R}\right)_{j} \times f_{\alpha}\left(E^{R}\right)_{j}
$$

where $\mathrm{N}_{c u t}^{M C}\left(E^{R}\right)_{j}$ is the number of MonteCarlo events after the cuts, and $f_{\alpha}\left(E^{R}\right)_{j}$ is the signal fraction.

Various distortions including reconstruction bias and detector resolution cause the reconstructed energy to be different from the true neutrino energy.

To correct or "unsmear" the reconstructed neutrino energy to reflect the true neutrino energy, an unsmearing matrix $\mathrm{U}_{i, j}$ is introduced. By definition, for a process $\alpha$, the unsmearing matrix connects the number of events in the $\mathrm{i}^{\text {th }}$ generated (true) energy bin to the number of events in the $\mathrm{j}^{\text {th }}$ reconstructed energy bin as follow:

$$
\sum_{j} U_{i, j}\left(N_{\alpha}^{M C}\right)_{j}=\left(N_{\alpha}^{M C}\right)_{i}
$$

The standard way to determine the unsmearing matrix is to first construct a smearing matrix $\mathrm{S}_{i, j}$ so that $\sum_{i} S_{j, i}\left(N_{\alpha}^{M C}\right)_{i}=\left(N_{\alpha}^{M C}\right)_{j}$, and then invert it to obtain $\mathrm{U}_{i, j}$. However, this method presents some challenges that arise from the difficulty of inverting sparse matrices. For this analysis, $\mathrm{U}_{i, j}$ is obtained from an iterative procedure and the details of the method are explained in [67]. 
Taking into account the unsmearing correction, the number of events of type $\alpha$ in the $i^{\text {th }}$ true energy bin can be written as:

$$
N_{\alpha, \text { cut }}\left(E^{T}\right)_{i}=\sum_{j} U_{i, j}^{\alpha} N_{\alpha, c u t}\left(E^{R}\right)_{j}
$$

For the MonteCarlo sample, equation (7.7) is true by construction, but for the data, the matrix $\mathrm{U}_{i, j}^{\alpha}$ is the best estimate of the proper unfolding.

The final correction needed to determine the true number of events comes from using the cut efficiency $\epsilon$ which is defined as:

$$
\epsilon_{i}=\frac{N_{\alpha, c u t}^{M C}\left(E_{\nu}\right)_{i}}{N_{\alpha}^{M C}\left(E_{\nu}\right)_{i}}
$$

Thus, the efficiency corrected number of events of type $\alpha$ in a given bin of true neutrino energy in the event sample is:

$$
N_{\alpha}\left(E^{T}\right)_{i}=\frac{1}{\epsilon_{i}} \times \sum_{j} U_{i, j}^{\alpha} f_{\alpha}\left(E^{R}\right)_{j} N_{c u t}\left(E^{R}\right)_{j}
$$

and for a given bin i, equation (7.4) becomes:

$$
\left(\frac{\sigma_{C C \pi^{+}}}{\sigma_{C C Q E}}\right)_{i}=\frac{\epsilon_{C C Q E} \times \sum_{j} U_{i, j}^{C C \pi^{+}} f_{C C \pi^{+}}\left(E^{R}\right)_{j} N_{C C \pi^{+} \text {cuts }}\left(E^{R}\right)_{j}}{\epsilon_{C C \pi^{+}} \times \sum_{j} U_{i, j}^{C C Q E} f_{C C Q E}\left(E^{R}\right)_{j} N_{C C Q E \text { cuts }}\left(E^{R}\right)_{j}}
$$

Equation (7.11) is the expression for the $\mathrm{CC} \pi^{+}$to CCQE cross section ratio. Before presentating the result in section 7.4 , we will discuss in section 7.2 the cuts used for selecting CCQE and $\mathrm{CC} \pi^{+}$events and in section 7.3 the sources of systematic errors.

\subsection{Event Selection}

For a given interaction, the event selection starts by requiring a specific number of subevents. Most $\mathrm{CC} \pi^{+}\left(\nu_{\mu}+p \rightarrow \mu^{-}+\pi^{+}+p\right)$ events have three subevents. The first subevent is the reaction itself. The second subevent comes from the Michel electron from the decay of the $\mu^{-}$. The third subevent is to due to the Michel 
electron from the $\mu^{+}$produced in the $\pi^{+}$decay. Most $\pi^{+}$will stop and decay at rest to a $4 \mathrm{MeV} \mu^{+}$that will not be visible, but will decay to a Michel electron that can be detected.

Most CCQE $\left(\nu_{\mu}+n \rightarrow \mu^{-}+p\right)$ events possess exactly two subevents. The first subevent comes from the interaction. The second is from the electron that follows the decay of the muon.

As explained above, both $\mathrm{CC} \pi^{+}$and CCQE processes involve electrons from muon decay in the final state. It is therefore important to ensure that the selected electrons are not due to a coincidence with a cosmic ray event in the beam window. For this reason, the number of PMT hits in the veto region is required to be less than six, while the number of PMT hits in the main tank for the primary event is required to be greater than 175 for $\mathrm{CC} \pi^{+}$interactions and 200 for CCQE interactions. To ensure that the events are contained and well reconstructed within the tank, the radius for each subevent is required to be less than $500 \mathrm{~cm}$. Finally, to improve the sample purity, both $\mathrm{CC} \pi^{+}$and $\mathrm{CCQE}$ event selections require a cut on the distance between the muon endpoint and the closest Michel vertex. In the case of both $\mathrm{CC} \pi^{+}$and $\mathrm{CCQE}$, the subevent topologies are distinct enough that one obtains an acceptable purity without using complex PID variables as used in the elastic scattering analysis.

\subsection{1 $\mathrm{CC} \pi^{+}$Event Selection}

The $\mathrm{CC} \pi^{+}$event sample for this analysis uses the following cuts:

- Exactly three sub-events

- First sub-event in beam window

- $\left(\mathrm{N}_{\text {veto }}\right)_{1}<6,\left(\mathrm{~N}_{\text {veto }}\right)_{2}<6,\left(\mathrm{~N}_{\text {veto }}\right)_{3}<6$ 
- $\left(\mathrm{N}_{\text {tank }}\right)_{1}>175,\left(\mathrm{~N}_{\text {tank }}\right)_{2}<200,\left(\mathrm{~N}_{\text {tank }}\right)_{3}<200$

- $\mathrm{R}_{1}<500 \mathrm{~cm}, \mathrm{R}_{2}<500 \mathrm{~cm}, \mathrm{R}_{3}<500 \mathrm{~cm}$.

- Michel distance $<150 \mathrm{~cm}$.

Note that the required number PMT hits in the tank for the first subevent is lower than the usual 200 hit requirement. This is because the actual Michel background ends around $\mathrm{N}_{\text {tank }} \sim 175$, lower than the $\mathrm{N}_{\text {tank }}>200$ cut used as a precautionary measure in the CCQE sample where the event toplogy is not as distinctive. This lower cut does not degrade the sample purity nor significantly increase the cosmic Michel background.

Table 7.1 shows the fractions of signal and background events passing the $\mathrm{CC} \pi^{+}$ event selection. The background events come primarily from events with multiple pions that lose one or more of them within the nucleus, or from CCQE events that acquire a $\pi^{+}$through hadronic interactions within the nucleus, so that the events contain a single $\mu^{-}$and $\pi^{+}$in the final state.

TABLE 7.1. MonteCarlo event compositions after the $\mathrm{CC} \pi^{+}$cut requirements.

\begin{tabular}{|c|r|}
\hline Process & Event Composition (\%) \\
\hline $\mathrm{CC} \pi^{+}$Resonant & 79.8 \\
\hline $\mathrm{CC} \pi^{+}$Coherent & 5.7 \\
\hline CCQE & 5.6 \\
\hline Multi-pion Resonant & 3.8 \\
\hline $\mathrm{CC} \pi^{0}$ & 1.6 \\
\hline DIS & 1.4 \\
\hline
\end{tabular}

\subsubsection{CCQE Event Selection}

The following cuts are used to select this sample:

- Exactly two sub-events

- First sub-event in beam window 
- $\left(\mathrm{N}_{\text {veto }}\right)_{1}<6,\left(\mathrm{~N}_{\text {veto }}\right)_{2}<6$

- $\left(\mathrm{N}_{\text {tank }}\right)_{1}>200,\left(\mathrm{~N}_{\text {tank }}\right)_{2}<200$

- Michel distance $<100 \mathrm{~cm}$.

- $\mathrm{R}_{1}<500 \mathrm{~cm}$

Table 7.2 shows the fractions of signal and background events passing the CCQE event selection. The background events come mostly from single pion events in which the pion is absorbed within the nucleus, so that the events contain a single $\mu^{-}$and no $\pi$ in the final state.

TABLE 7.2. MonteCarlo event compositions after the CCQE cut requirements.

\begin{tabular}{|c|r|}
\hline Process & Event Composition (\%) \\
\hline CCQE & 78 \\
\hline $\mathrm{CC} \pi^{+}$Resonant & 18.3 \\
\hline $\mathrm{CC} \pi^{+}$Coherent & 1.1 \\
\hline $\mathrm{NC} \pi^{0}$ & 2.0 \\
\hline Multi-pion Resonant & 0.4 \\
\hline
\end{tabular}

\subsection{Systematic Errors}

The systematic uncertainties in this analysis are divided into two parts. The first part contains the uncertainties due to the neutrino flux prediction and the neutrino cross section, while the second part is composed of the uncertainties due to the optical model. The optical model refers to the properties of light generation and transmission in the detector mineral oil. This separation comes from the fact that two different techniques are used to calculate the different terms in the error matrix as defined in equation (7.11).

$$
\begin{aligned}
M_{i j} & =M^{\pi^{+} \text {flux }}+M^{\pi^{-} \text {flux }}+M^{K+\text { flux }}+M^{K_{L}^{0} \text { flux }}+M^{\text {beam }} \\
& +M^{X \text { sec }}+M^{N C \pi^{0} \text { production }}+M^{\text {Dirt }}+M^{\text {optical model }}
\end{aligned}
$$


Each term in the error matrix represents a covariance matrix obtained from varying simultaneously all the parameters involved in modelling this contribution to the event simulation by a random amount derived from a Gaussian distribution with mean zero and sigma one. The simulations obtained from these variations of the parameters will be referred as multisims.

For the uncertainties due to the neutrino flux prediction and the neutrino cross section, the multisims can be constructed by an event-by-event reweighting mechanism of a single MonteCarlo sample to reduce the necessary computation. For the optical model errors however, this reweighting method could not be used due to the effect on the energy distribution when the optical model parameters are varied. Thus, the optical model multisims were produced by generating 66 fully reconstructed hit-level simulations which where then passed through the selection cuts for the various samples.

\subsubsection{Cross Section and Flux Uncertainties}

The two largest contributions to this part of the error matrix come from uncertainties in the cross sections and in the rate of $\pi^{+}$production in the target. The latter represents the fact that most interacting neutrinos come from these pions. Figure 7.1 shows the cross section ratio with the cross section and flux uncertainties.

\subsubsection{Optical Model Uncertainties}

To calculate the optical model errors we reprocessed the complete Monte Carlo sample of each optical model variation to first obtain the efficiencies, the signal fractions, and the unsmearing matrices, and then determined the $\mathrm{CC} \pi^{+}$to $\mathrm{CCQE}$ ratio for that variation. The left panel of figure 7.2 shows the cross section ratio with optical model errors only. The right panel is the fractional error in the ratio 

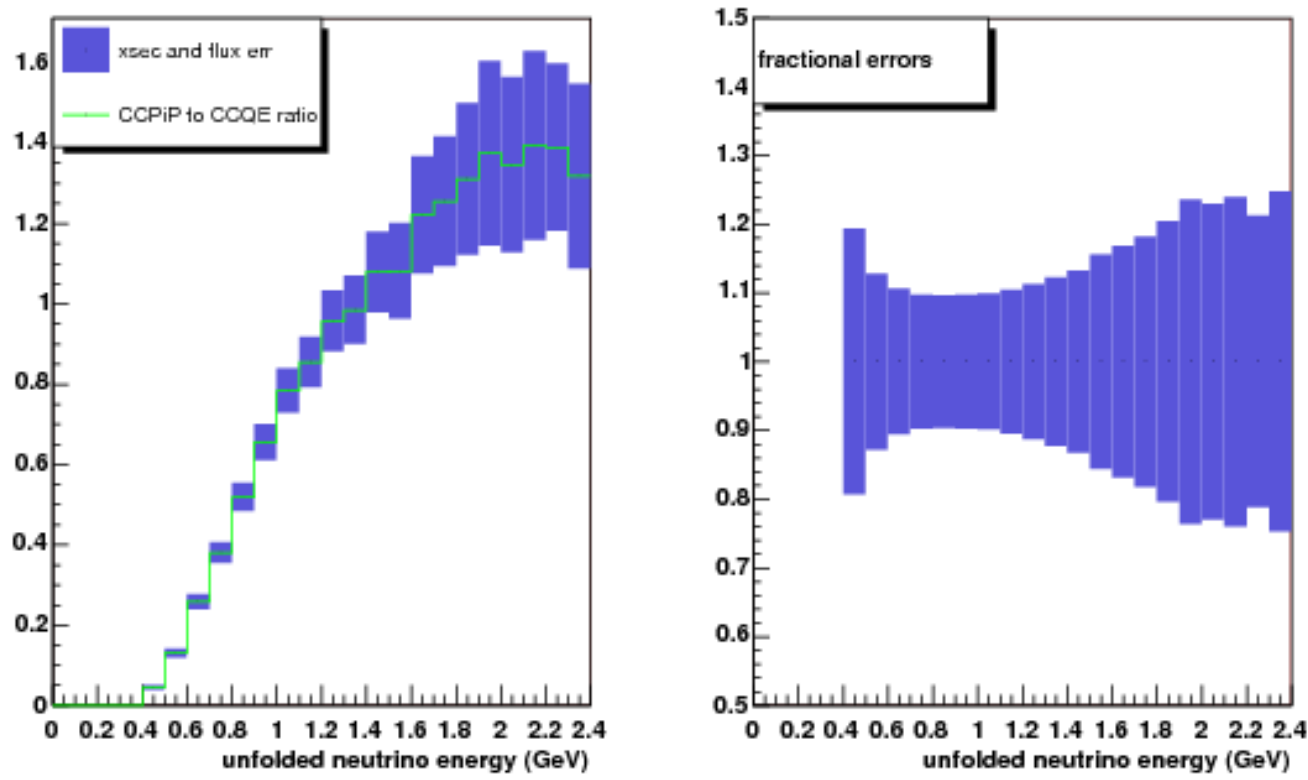

FIGURE 7.1. Right:The CC $\pi^{+}$to CCQE ratio with cross section and flux uncertainties. Left: Ratio fractional error from cross section and flux uncertainties.

due to the optical model uncertainties only. The fractional errors are large at low energy because in this energy range particles produced less light to be detected .

\subsection{Cross Section Ratio Measurement}

\subsubsection{Result Overview}

An initial analysis of the cross section ratio was presented in 2006 [68] when only the Stancu fitter was available. Since then, the P-fitter was developed, but most importantly, significant improvements were made in the Monte Carlo optical model simulations and the CCQE event selection. For the analysis presented in this thesis, we will be using the latest version of our Monte Carlo simulation as well as both the Stancu fitter and the P-fitter to independently check our result. Note that the P-fitter, as explained in chapter 4 , has the potential to reconstruct events under one track or two tracks hypotheses. Here, only the one track hypothesis will be 

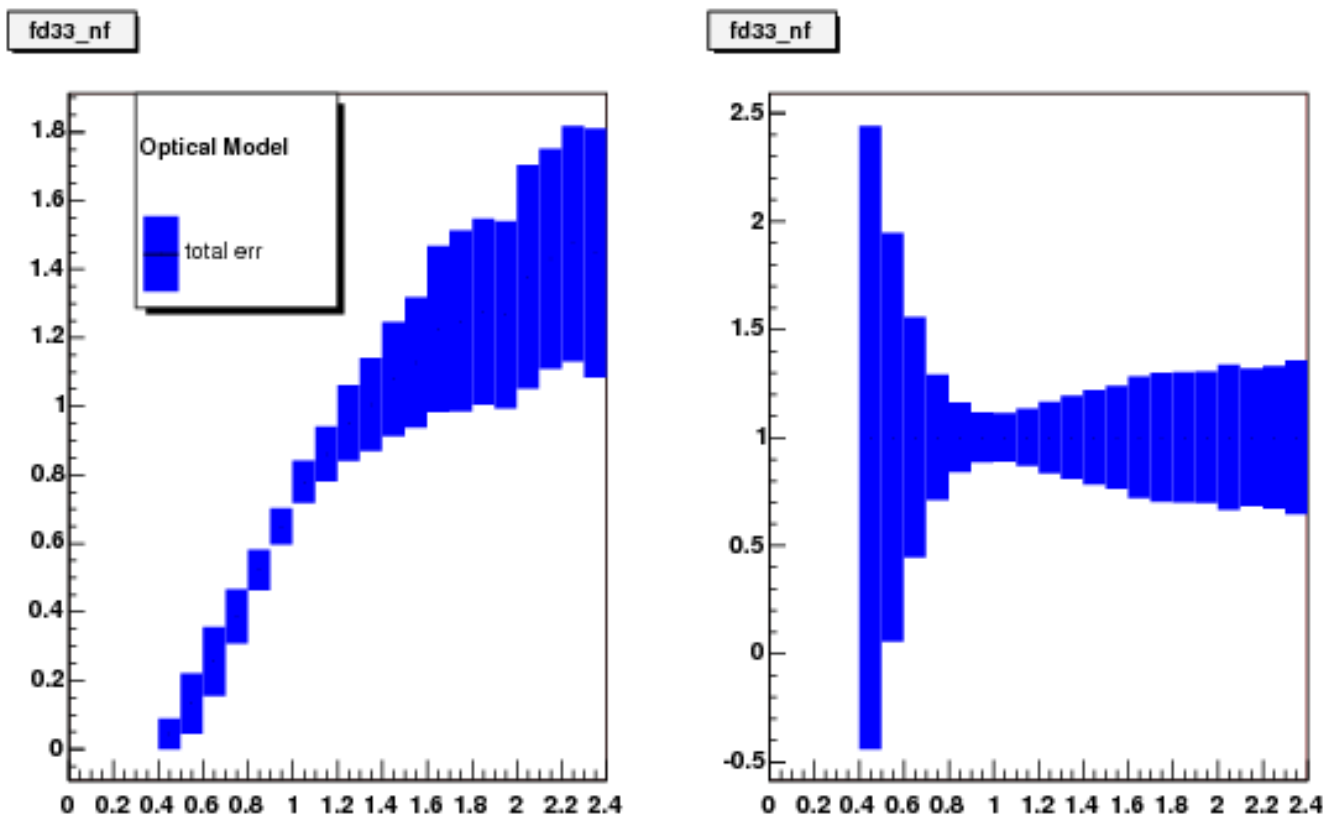

FIGURE 7.2. Left: The CC $\pi^{+}$to CCQE cross section ratio with optical model uncertainties only. Right: The ratio fractional errors from the optical model only.

used, and therefore we will be referring to the P-fitter as the one-track fitter with the assumption that the track is a muon.

Before submitting the ratio measurement for publication, the MiniBooNE collaboration thought it was important to verify that the two fitters gave consistent results. The procedure including event selections and calculation of the event rates, is the same for both fitters. However, the event composition is different for each fitter, and therefore the result of the ratio measurement and the systemtic errors are computed accordingly. LSU was responsible for the $\mathrm{CC} \pi^{+} / \mathrm{CCQE}$ ratio measurement using the P-fitter, and this result is the focus of the following section.

\subsubsection{Results}

The one-track fitter (P-fitter) was found to overestimate the reconstructed muon energy because of the extra charge created primarily by the pion. This problem is not found in the Stancu fitter because it reconstructs the muon energy from the 
charge in the muon ring. To correct for the effect of the extra charge, the muon energy in the one-track fitter was recalibrated so that it was closer to the true visible energy of the muon. The details of this procedure are given in [67]. Results from the one-track fitter, before (1track-old) and after (1track-new) the energy adjustement as well as results from the Stancu fitter are presented.
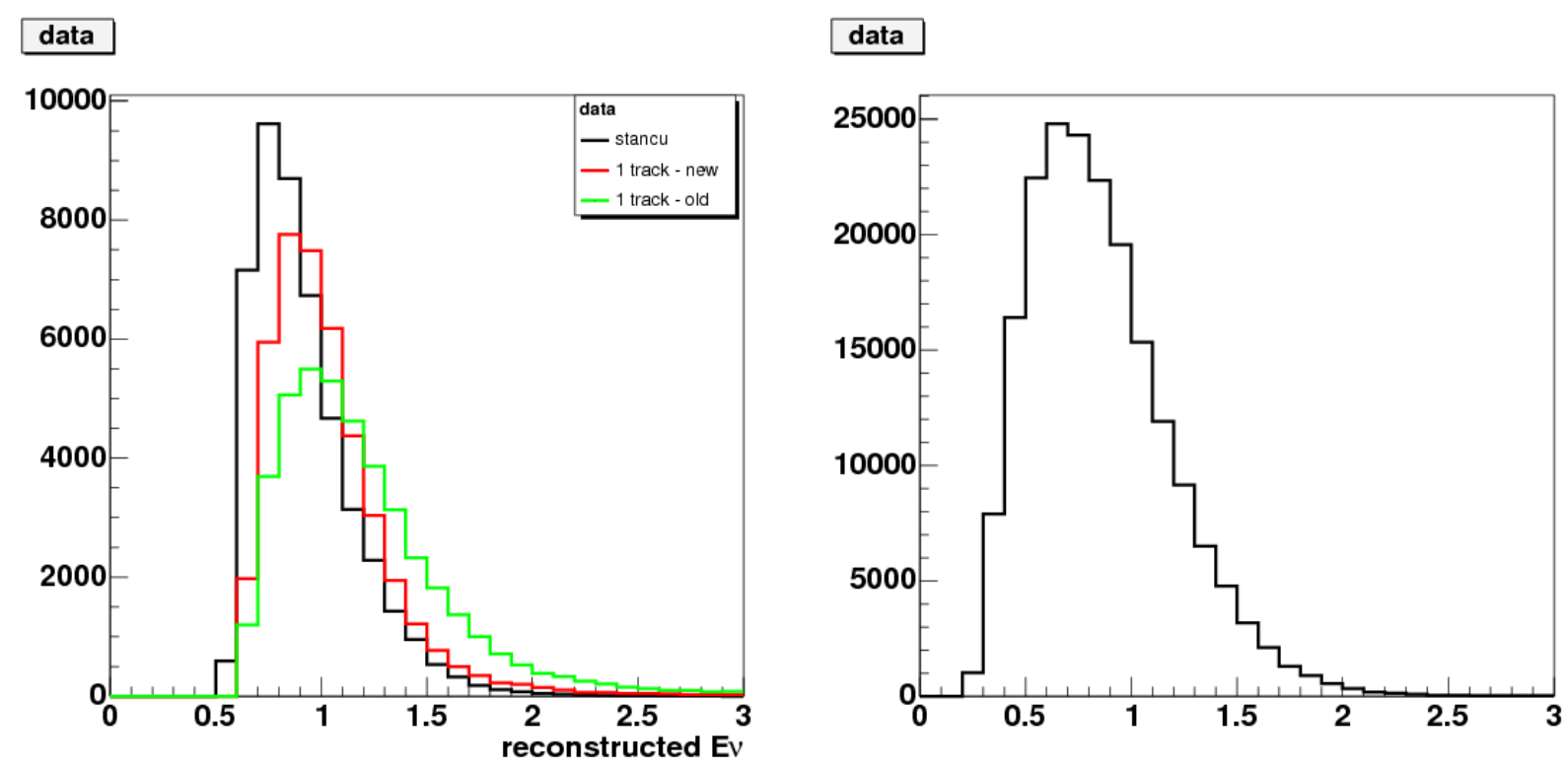

FIGURE 7.3. Reconstructed neutrino energy for neutrino mode data for the $\mathrm{CC} \pi^{+}$(left) and CCQE (right). For CC $\pi^{+}$the Stancu fitter (black) is compared with two versions of one-track fitter, original (old - green) and with muon energy adjustment (new - red).

In figure 7.3 , the reconstructed neutrino energy distributions for both $\mathrm{CC} \pi^{+}$ (left) and CCQE data samples (right) are shown. The most significant difference between reconstructed neutrino energy distributions for the $\mathrm{CC} \pi^{+}$sample in the figure is that between the Stancu fitter and the original one-track fitter (1trackold). The signal fraction and cut efficiency for $\mathrm{CC} \pi^{+}$events reconstructed with the three different fitters are compared in figure 7.4. It is important to notice that the signal fraction for the one-track fitter is higher than that for the Stancu fitter. The difference in the cut efficiency between the Stancu fitter and the onetrack fitters comes from the fact that more events are reconstructed with the 
first one. The unsmearing matrices for the three reconstructions of the $\mathrm{CC} \pi^{+}$ sample are presented in figure 7.5. From this figure we can see the reconstructed neutrino energy distribution for the corrected one-track fitter has a shape close to the generated energy distribution, and the unsmearing matrix is mostly diagonal, while for the other two cases we can see high off-diagonal elements.

Finally, in figure 7.6 the top plot compares the $\mathrm{CC} \pi^{+}$to $\mathrm{CCQE}$ ratios for all fitters. The difference between the results is negligible and we can draw the conclusion that the analysis in general and the unsmearing procedure in particular are not sensitive to the reconstruction scheme used. The left plot on the bottom of figure 7.6 shows the $\mathrm{CC} \pi^{+}$to CCQE ratio measurement using the P-fitter with all the errors (left plot) while the right plot shows the fractional error for neutrino energy between $0.4 \mathrm{GeV}$ and $2.4 \mathrm{GeV}$.

\subsubsection{Summary}

We have presented a measurement of the $\mathrm{CC} \pi^{+}$to $\mathrm{CCQE}$ cross section ratio on a carbon nuclear target near $1 \mathrm{GeV}$. The analysis was done with two different reconstruction fitters and their result seem to agree well. The results from this analysis will be submitted for publication. My contribution in this analysis was to measure the ratio using the one-track fitter. 

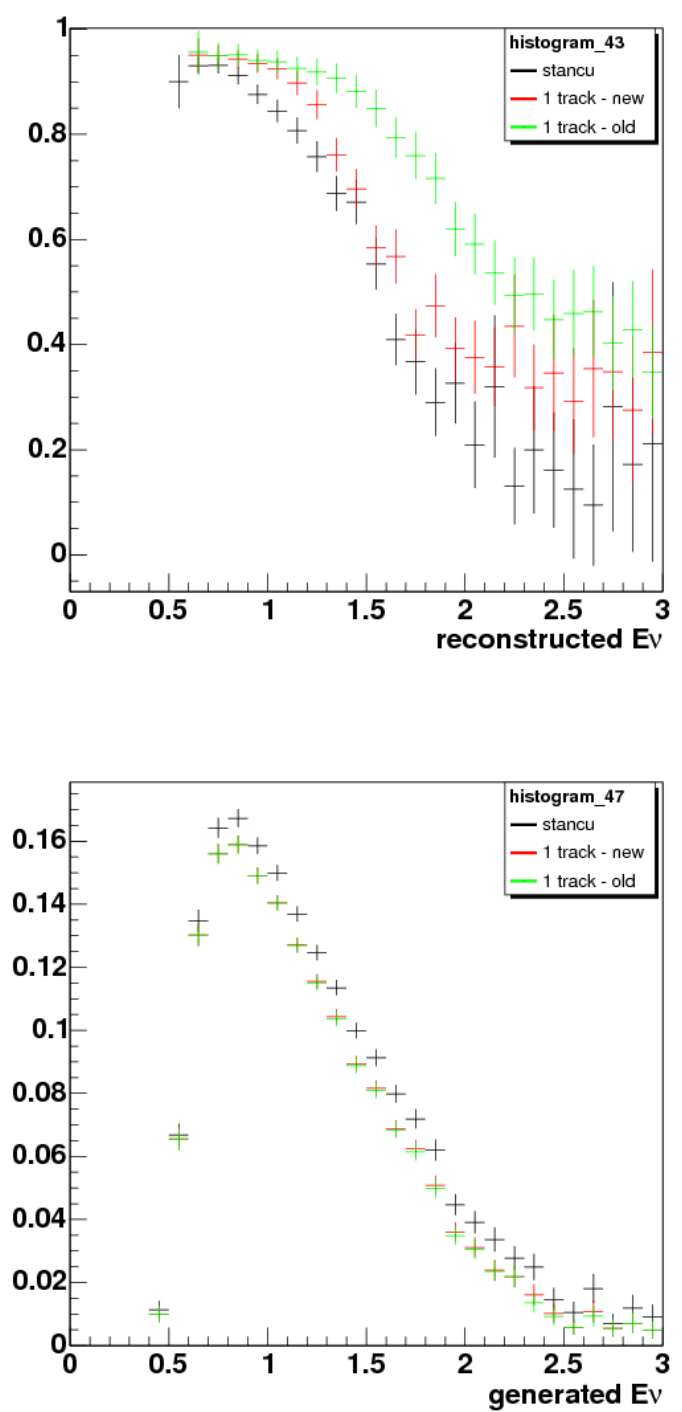

FIGURE 7.4. The signal fraction (left) and cut efficiency (right) for the $\mathrm{CC} \pi^{+}$sample for three fitters. 

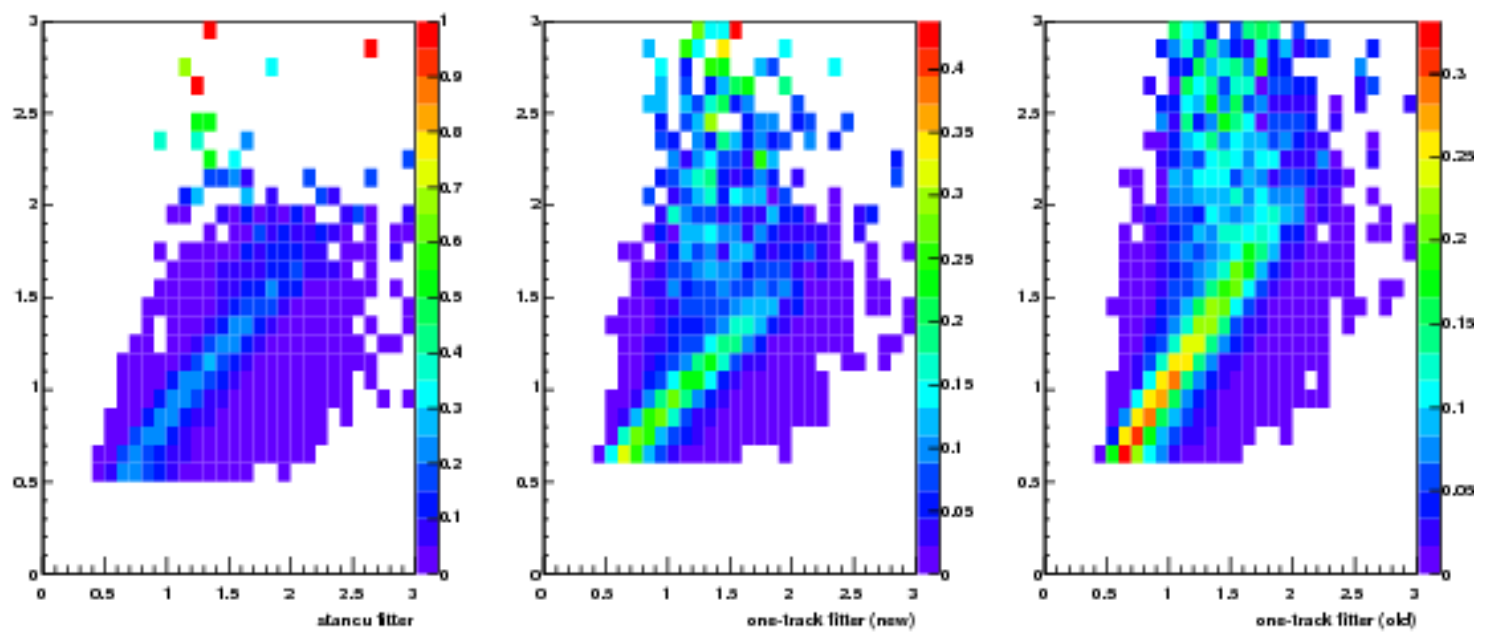

FIGURE 7.5. The unsmearing matrices for the three fitters 

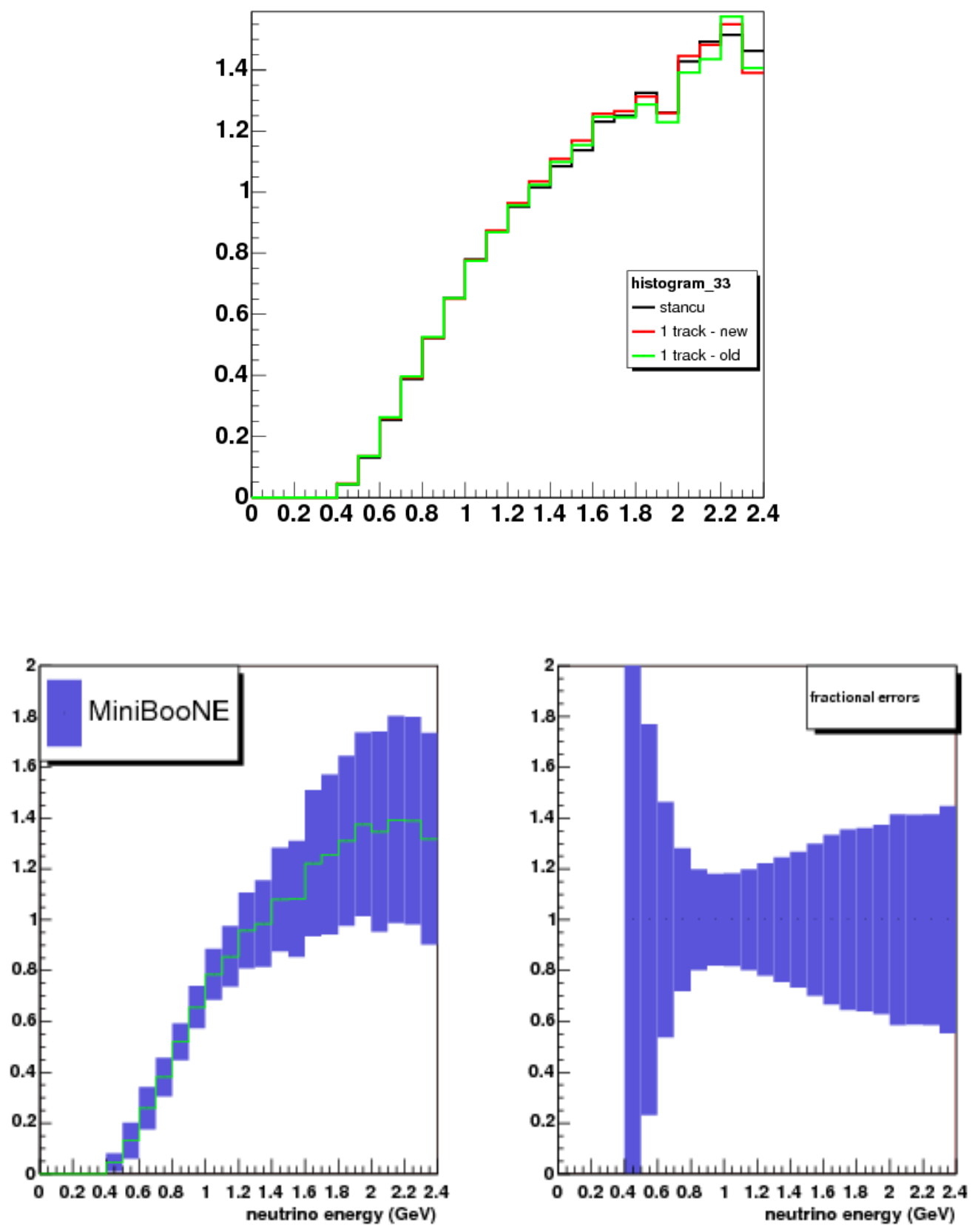

FIGURE 7.6. Top: The CC $\pi^{+}$to CCQE ratio for stancu fitter (black curve), the P-fitter without energy correction (green) and with energy correction (red). Bottom: the left plot is the $\mathrm{CC} \pi^{+}$to CCQE ratio distribution using the P-fitter including all the sources of errors. The right plot shows the fractional errors after taking into account all sources of errors. 


\section{Chapter 8 \\ Conclusion}

In this thesis, we discussed three different analyses. The focus of the first one was to search for the effects of a neutrino magnetic moment on $\nu_{\mu}+e \rightarrow \nu_{\mu}+e$ interactions at low electron energy. The $\nu_{\mu}$ e events were selected by applying a set of cuts on basic variables and on PID variables constructed for the purpose of separating signal events from background events, mostly Michel electrons from the decay of cosmic rays muons. After all cuts were applied, we measured 51 data events.

Two different and independent methods were used to determine the number of data events due to elastic scattering. The first method is a counting experiment which uses the number of simulated beam events and the number of beam off events, obtained from the strobe event sample passing all the cuts. These numbers are added and normalized to the data protons-on-target (POT) to determine the expected number of events. From this method, the predicted number of events is $32.8 \pm 3.4$ (stat) \pm 3.8 (syst) for an excess of $18.2 \pm 8.8$ events.

The second method is based on an unbinned maximum likelihood estimate that uses the peaking property of the the angular distribution of the $\nu_{\mu}$ e events and the flatness of the background events in $\cos \theta$ to directly determine the number of $\nu_{\mu} \mathrm{e}$ events. Using the shape of the angular distribution obtained from a sample of simulated elastic scattering events, a negative log-likelihood function was constructed. The number of signal events obtained from minimizing this negative log-likelihood function is $15.3 \pm 6.6$ (stat) \pm 4.1 (syst), consistent with the counting method. 
Since the fitting procedure does not rely on the Monte Carlo as much as the counting experiment, it was used to set a limit on the neutrino magnetic moment. With only 4.6 events in the data sample predicted to be due to the weak interaction, the observed excess led us to deduce that $\mu_{\nu}<12.7 \times 10^{-10} \mu_{B}$ at the $90 \%$ CL which is higher than the current best limit of $\mu_{\nu}<6.8 \times 10^{-10} \mu_{B}$ set by LSND [18].

The second analysis presented in this thesis is the study of the $\nu_{\mu}$ e events at high energy. The motivation for this analyis is to determine if the excess observed at low energy extends to the higher energy region. After precuts on basic variables, two different methods are used to conduct the analysis. The first method is based on cuts applied on PID variables from the Boosting Decision Tree algorithm while the second method is based on cuts applied on PID variables from the Track-Based Analysis algorithm. From the expected number of events, which is the counting experiment outlined earlier, both methods show an excess of events but not as large as the excess observed at low energy. Although the excess at high energy is less significant than the excess at low energy, it is important to note that we obseve an excess for each $\cos \theta$ range for both methods.

The subject of the third analysis is the measurement of the cross section for single charged pion production $\left(\mathrm{CC} \pi^{+}\right)$relative to the charged current quasi-elastic (CCQE) cross section as a function of the neutrino energy. The result of this analysis is based on more than $46000 \mathrm{CC} \pi^{+}$interactions with neutrino energy between $0.3 \mathrm{GeV}$ and $2.4 \mathrm{GeV}$. MiniBooNE's $\mathrm{CC} \pi^{+}$event sample is the world largest in this energy range, and is about 10 times larger than the samples of all previous experiments combined.

The cross section ratio analysis was conducted with two different reconstruction fitter algorithms, and the results from both fitters are consistent with each other and with the Monte Carlo prediction based on the Rein and Sehgal model. As 
shown in figure 8.1, our results are also consistent with previous experimental results from K2K and ANL [69, 23, 24].

A MiniBooNE technical note on this analyis is being reviewed by the collaboration. Once approved, a paper based on the note will be submitted for publication.

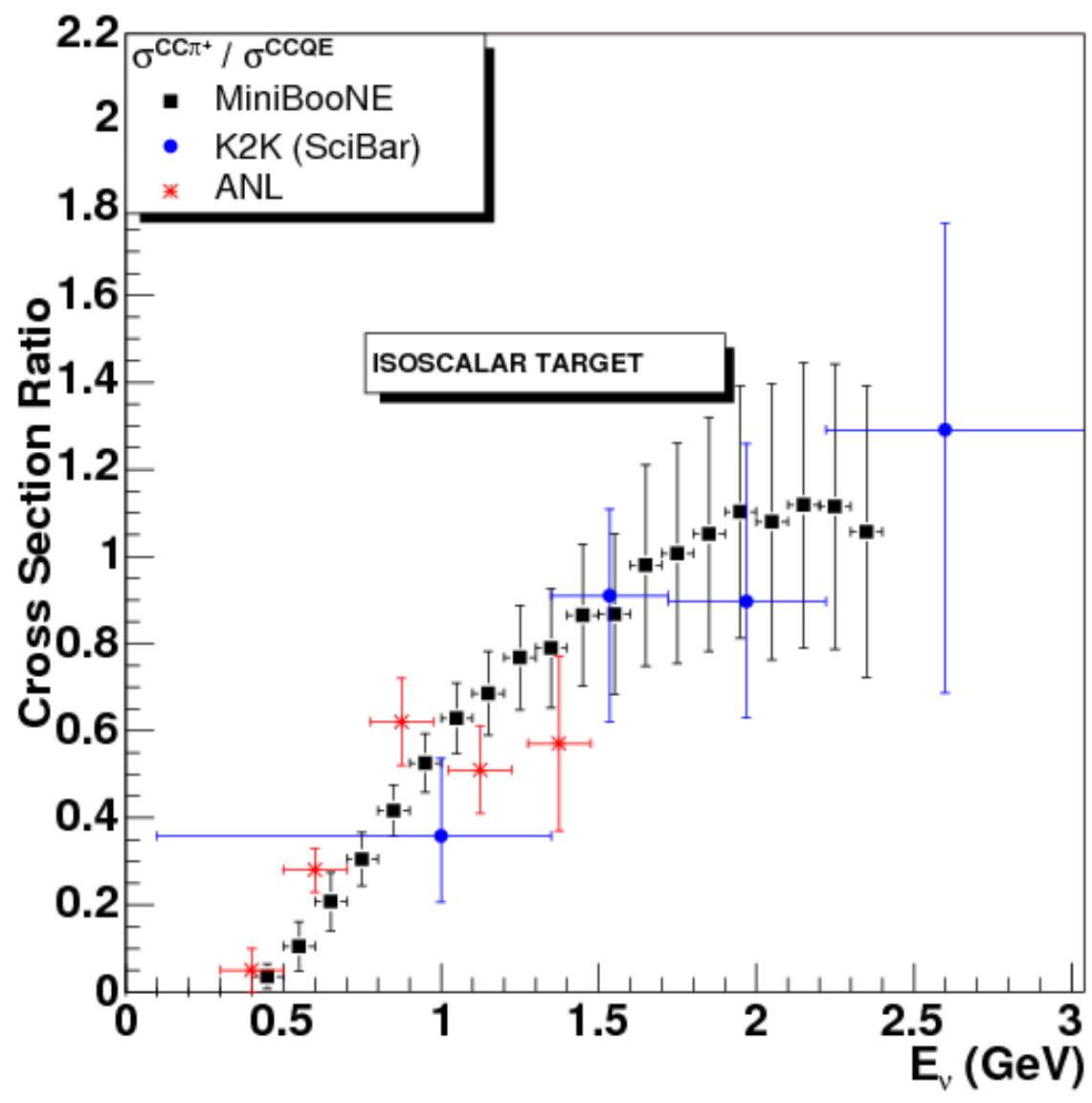

FIGURE 8.1. The $\mathrm{CC} \pi^{+}$to CCQE ratio for MiniBooNE, K2K and ANL 


\section{References}

[1] E. Fermi, Nuovo Cim. 11, 1 (1934).

[2] S. L. Glashow, Nucl. Phys. 22, 579 (1961).

[3] S. Weinberg, Phys. Rev. Lett. 19, 1264 (1967).

[4] R. C. Allen et al., Phys. Rev. D 43, R1 (1991).

[5] J. Dorenbosch et al., Z. Phys. C41, 567 (1989).

[6] P. Vilain et al., Phys. Lett. B332, 465 (1994).

[7] L. A. Ahrens et al., Phys. Rev. D 41, 3297 (1990).

[8] J. F. Beacom and P. Vogel, Phys. Rev. Lett. 83, 5222 (1999).

[9] B. W. Lee and R. E. Shrock, Phys. Rev. D 16, 1444 (1977).

[10] J. Liu, Phys. Rev. D 35, 3447 (1987).

[11] A. Suzuki, M. Mori, K. Numata, and Y. Oyama, Phys. Rev. D 43, 3557 (1991).

[12] G. L. Fogli et al., Phys. Rev. D 67, 073002 (2003).

[13] S. Dodelson, G. Gyuk, and M. S. Turner, Phys. Rev. D 49, 5068 (1994).

[14] G. G. Raffelt, Phys. Rev. Lett. 64, 2856 (1990).

[15] J. M. Lattimer and J. Cooperstein, Phys. Rev. Lett. 61, 23 (1988).

[16] R. Barbieri and R. N. Mohapatra, Phys. Rev. Lett. 61, 27 (1988).

[17] D. W. Liu et al., Phys. Rev. Lett. 93, 021802 (2004).

[18] L. B. Auerbach et al., Phys. Rev. D 63, 112001 (2001).

[19] F. Reines, H. S. Gurr, and H. W. Sobel, Phys. Rev. Lett. 37, 315 (1976).

[20] H. T. Wong et al., Phys. Rev. D75, 012001 (2007).

[21] A. A. Aguilar-Arevalo et al., Physical Review Letters 100, 032301 (2008).

[22] S. Chekanov et al., Phys. Rev. D70, 052001 (2004).

[23] S. J. Barish et al., Phys. Rev. D 19, 2521 (1979).

[24] T. Kitagaki et al., Phys. Rev. D 34, 2554 (1986). 
[25] C. Athanassopoulos et al., Phys. Rev. C54, 2685 (1996).

[26] C. Athanassopoulos et al., Phys. Rev. Lett. 81, 1774 (1998).

[27] F. Boehm et al., Phys. Rev. D 64, 112001 (2001).

[28] M. Apollonio et al., Phys. Lett. B466, 415 (1999).

[29] B. Armbruster et al., Phys. Rev. D 65, 112001 (2002).

[30] E. D. Church, K. Eitel, G. B. Mills, and M. Steidl, Phys. Rev. D 66, 013001 (2002).

[31] A. A. Aguilar-Arevalo et al., Physical Review Letters 98, 231801 (2007).

[32] A. A. Aguilar-Arevalo et al., The miniboone detector, 2008, arXiv.org:0806.4201 (has been submitted to Nucl. Instr. Meth. A).

[33] B. T. Fleming, FERMILAB-THESIS-2001-02.

[34] A. A. Aguilar-Arevalo et al., The neutrino flux prediction at miniboone, 2008, arXiv.org:0806.1449, (has been submitted to Phys. Rev. D.).

[35] R. Patterson, The PMT Charge Calculation, MiniBooNE Technical Note 83 (2003).

[36] R. Imlay, B. Metcalf, G. Schofield, M. Sung, and M. Wascko, Design and Commissioning of the Muon Tracker, MiniBooNE Technical Note 98 (2003).

[37] R. Imlay, B. Metcalf, S. Ouedraogo, M. Sung, and M. Wascko, Energy Calibration of Stopping Muons in MiniBooNE Using the Muon Tracker and Cubes, MiniBooNE Technical Note 106 (2003).

[38] R. Imlay, B. Metcalf, S. Ouedraogo, M. Sung, and M. Wascko, Commissioning the Scintillating Cubes, MiniBooNE Technical Note 105 (2003).

[39] A. A. Aguilar-Arevalo, FERMILAB-THESIS-2008-01.

[40] S. Agostinelli et al., Nucl. Instrum. Meth. A506, 250 (2003).

[41] D. Casper, Nucl. Phys. Proc. Suppl. 112, 161 (2002).

[42] CERN Program Library Long Writeup W5013 (1993).

[43] I. Chemakin et al., Phys. Rev. C77, 015209 (2008).

[44] J. R. Sanford and C. L. Wang, Ags internal reports 11299 and 11479, Brookhaven National Laboratory, 1967, (Unpublished).

[45] M. G. Catanesi et al., Physical Review C (Nuclear Physics) 77, 055207 (2008). 
[46] J. R. Monroe, FERMILAB-THESIS-2006-44.

[47] T. Abbott et al., Phys. Rev. D 45, 3906 (1992).

[48] T. Eichten et al., Nucl. Phys. B44, 333 (1972).

[49] P. A. Piroué and A. J. S. Smith, Phys. Rev. 148, 1315 (1966).

[50] L. Bugel and M. Sorel, Magnetic Field Measurements for the MiniBooNE Prototype Horn, MiniBooNE Technical Note 34 (2001).

[51] M. Sorel, FERMILAB-THESIS-2005-07.

[52] K. Zuber, Neutrino Physics, volume 1st Ed., London:Institute of Physics Publishing, 2004.

[53] C. H. Llewellyn Smith, Phys. Rept. 3, 261 (1972).

[54] D. Rein and L. M. Sehgal, Ann. Phys. 133, 79 (1981).

[55] R. P. Feynman, M. Kislinger, and F. Ravndal, Phys. Rev. D3, 2706 (1971).

[56] D. Toptygin, Time-Resolved Fluorecence of MiniBooNE Mineral Oil - Report 1, MiniBooNE Technical Note 122 (2004).

[57] R. B. Patterson, FERMILAB-THESIS-2007-19.

[58] I. Stancu, An Introduction to the Maximum Likelihood Event Reconstruction in MiniBooNE, MiniBooNE Technical Note 50 (2002).

[59] J. L. Raaf, FERMILAB-THESIS-2005-20.

[60] F. James and M. Roos, "MINUIT", A System For Function Minimization and Analysis of the Parameter Errors and Correlations, (1975).

[61] I. Stancu, Maximum Likelihood Event Reconstruction for BooNE: Point-like Particles in a Perfect Detector, MiniBooNE Technical Note 6 (1998).

[62] Y. Liu and I. Stancu, Toward the MiniBooNE Charge Likelihoods, MiniBooNE Technical Note 100 (2003).

[63] H.-J. Y. B. Roe, Brief Manual for Using Boost Programs, MiniBooNE Technical Note 123 (2004).

[64] D. C. Cox, FERMILAB-THESIS-2008-08.

[65] A. Green, Private Communication, (2003).

[66] G. Garvey et al., Anomalous Production of Events in the Forward Direction, MiniBooNE Technical Note 235 (2007). 
[67] S. L. et al., $C C \pi^{+}$ratio, MiniBooNE Technical Note 256 (2008).

[68] M. O. Wascko, Nucl. Phys. Proc. Suppl. 159, 50 (2006).

[69] and others, (2008). 


\section{Vita}

Serge Aristide Ouedraogo was born in February 1975, in Ouagadougou, Burkina Faso. He graduated from high school in 1993 from Lycee Marien N'Gouabi after which he attended a year at the University of Ouagadougou before going to Ghana where he stayed until 1995.

In 1996 he moved to the United States of America where he attended the University of Arkansas at Little Rock (UALR). At UALR, he received the prestigious Donaghey Scholar Award in 1998 and in the spring of 2001, he graduated with honors from the Department of Physics and Astronomy with a Bachelor degree of Science.

In 2001 he was awarded a teaching assistanship from Louisiana State University (LSU) where he began his graduate studies in the fall of the same year. In December 2003, he received his master degree in physics, and in the spring of 2004 he joined the experimental high energy physics to work with Prof. William J. Metcalf and Richard L. Imlay.

In the summer of 2004, he moved to Fermilab to work on the Mini Booster Neutrino Experiment (MiniBooNE). He stayed at Fermilab for the next three years before moving back to LSU after the publication of first MiniBooNe neutrino oscilation result in 2007. In the fall of 2008, he received the degree of Doctor of Philosophy in experimental physics for his work on: "A Measurement of the Muon Neutrino Magnetic moment And A Measurement of the Cross Sections Ratio of Charged Current Single Pion Production $\left(\mathrm{CC} \pi^{+}\right)$to Charged Current Quasi Elastic (CCQE) “. 